Prepared for the U. S. Department of Energy

under Contract DE-AC05-76RL01830

\title{
MANGO - Modal Analysis for Grid Operation: A Method for Damping Improvement through Operating Point Adjustment
}

$\begin{array}{ll}\text { Z Huang } & \text { R Diao } \\ \text { N Zhou } & \text { JC Fuller } \\ \text { FK Tuffner } & \text { WA Mittelstadt } \\ \text { Y Chen } & \text { JF Hauer } \\ \text { DJ Trudnowski } & \text { JE Dagle }\end{array}$

October 2010

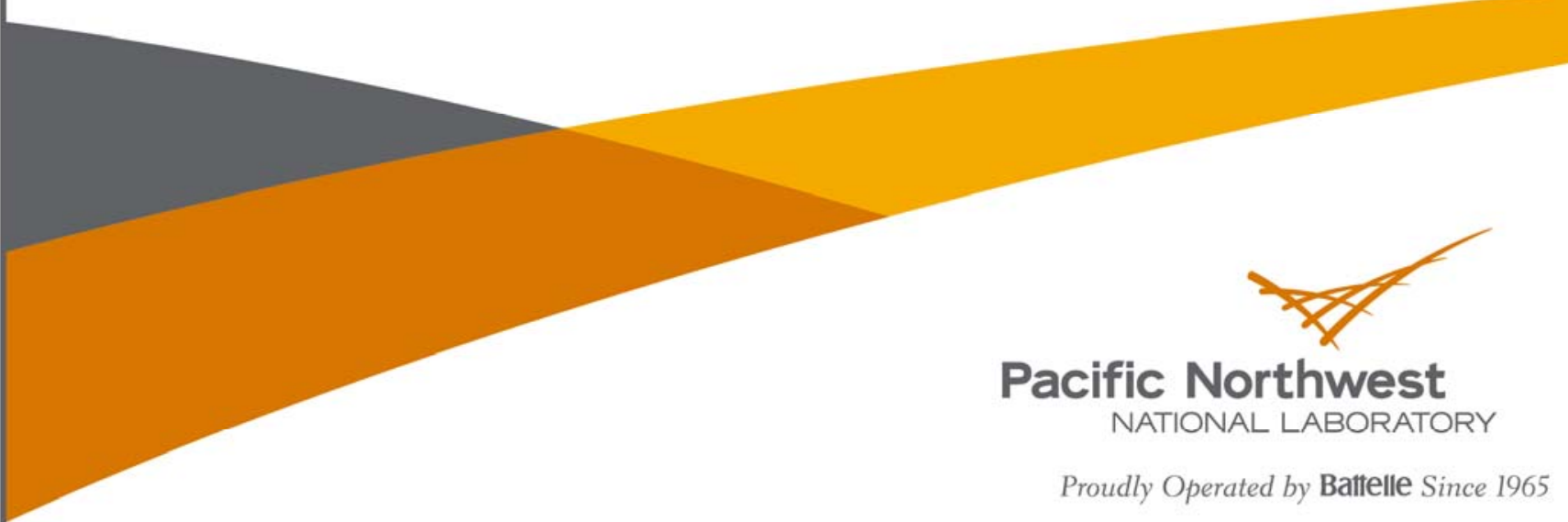




\title{
DISCLAIMER
}

This report was prepared as an account of work sponsored by an agency of the United States Government. Neither the United States Government nor any agency thereof, nor Battelle Memorial Institute, nor any of their employees, makes any warranty, express or implied, or assumes any legal liability or responsibility for the accuracy, completeness, or usefulness of any information, apparatus, product, or process disclosed, or represents that its use would not infringe privately owned rights. Reference herein to any specific commercial product, process, or service by trade name, trademark, manufacturer, or otherwise does not necessarily constitute or imply its endorsement, recommendation, or favoring by the United States Government or any agency thereof, or Battelle Memorial Institute. The views and opinions of authors expressed herein do not necessarily state or reflect those of the United States Government or any agency thereof.

\author{
PACIFIC NORTHWEST NATIONAL LABORATORY \\ operated by \\ BATTELLE \\ for the \\ UNITED STATES DEPARTMENT OF ENERGY \\ under Contract DE-AC05-76RL01830
}

Printed in the United States of America

\author{
Available to DOE and DOE contractors from the Office of Scientific and Technical \\ Information, \\ P.O. Box 62, Oak Ridge, TN 37831-0062; ph: (865) 576-8401 fax: (865) 576-5728 email: \\ reports@adonis.osti.gov
}

\author{
Available to the public from the National Technical Information Service, \\ U.S. Department of Commerce, 5285 Port Royal Rd., Springfield, VA 22161 \\ ph: (800) 553-6847 fax: (703) 605-6900 \\ email: orders@ntis.fedworld.gov online ordering: http://www.ntis.gov/ordering.htm
}

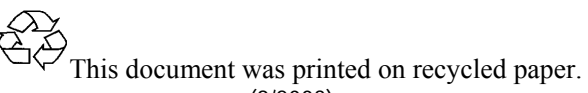

$(9 / 2003)$ 


\title{
MANGO - Modal Analysis for Grid Operation: A Method for Damping Improvement through Operating Point Adjustment
}

\author{
Z Huang \\ R Diao \\ N Zhou \\ JC Fuller \\ FK Tuffner \\ WA Mittelstadt ${ }^{1}$ \\ Y Chen \\ JF Hauer \\ DJ Trudnowski ${ }^{2}$ \\ JE Dagle
}

October 2010

Prepared for

the U. S. Department of Energy

under Contract DE-AC05-76RL01830

Pacific Northwest National Laboratory

Richland, Washington 99352

\footnotetext{
${ }^{1}$ Western Electricity Coordinating Council

${ }^{2}$ Montana Tech of the University of Montana
} 



\section{Executive Summary}

Small signal stability problems are one of the major threats to grid stability and reliability in the U.S. power grid. An undamped mode can cause growing oscillations and may result in system breakups and large-scale blackouts. There have been several incidents of system-wide oscillations. Of those incidents, the most notable is the August 10, 1996 western system breakup, a result of undamped system-wide oscillations. Significant efforts have been devoted to monitoring system oscillatory behavior from measurements in the past 20 years. The deployment of phasor measurement units (PMU) provides highprecision, time-synchronized data needed for detecting oscillation modes. Measurement-based modal analysis, also known as ModeMeter, uses real-time phasor measurements to identify system oscillation modes and their damping. Low damping indicates potential system stability issues. Modal analysis has been demonstrated with phasor measurements to have the capability of estimating system modes from oscillation signals, probing data, and ambient data.

With more and more phasor measurements available and ModeMeter techniques maturing, there is yet a need for methods to bring modal analysis from monitoring to actions. The methods should be able to associate low damping with grid operating conditions, so operators or automated operation schemes can respond when low damping is observed. The work presented in this report aims to develop such a method and establish a Modal Analysis for Grid Operation (MANGO) procedure to provide recommended actions (such as generation re-dispatch or load reduction), and aid grid operation decision making for mitigating inter-area oscillations. This project directly contributes to the Department of Energy Transmission Reliability Program's goal of "improving reliability of the nation's electricity delivery infrastructure."

The fundamental part of the work explores the relationship between low damping and grid operating conditions to develop recommended actions for damping improvement, and therefore reduce the chance of system breakup and power outages. Different from power system stabilizers and other modulation control mechanisms, MANGO improves damping through operating point adjustments. Traditionally, the modulation-based methods do not change the system's operating point, but improve damping through automatic feedback control. Figure S-1 illustrates the difference of these two types of damping improvement methods. MANGO, represented in red, and modulation control, represented in magenta, are complementary towards the same goal.

MANGO is a measurement-based procedure, as shown in Figure S-2. As the first stage of development, the MANGO procedure is targeted to have operators in the loop. Practical implementation is envisioned to be achieved by integrating MANGO recommendations into existing operating procedures. The MANGO model can be updated according to the current measurement and mode estimation results. Operators are included into the loop to bring in expert knowledge. In the future, after the confidence and accuracy of the MANGO model is built up, it is expected that the automatic closed-loop control will be introduced to expedite the implementation and avoid human errors. The automatic process can be integrated into a remedial action scheme (RAS) system or a special protection system (SPS). 


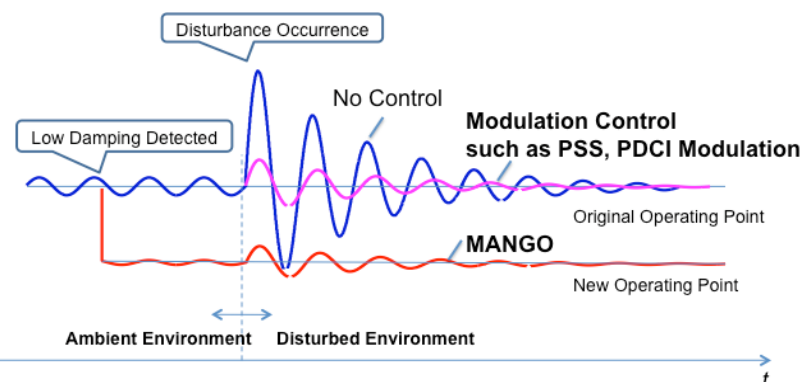

Figure S-1. MANGO versus modulation control

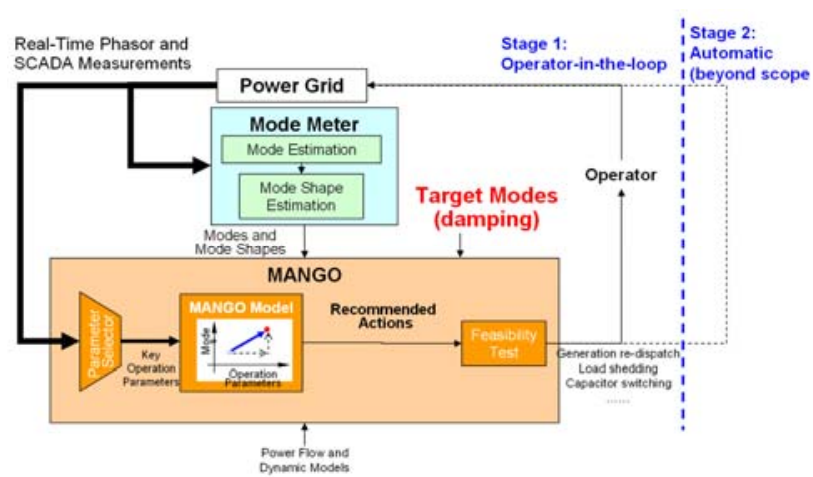

Figure S-2. MANGO framework

Extensive sensitivity studies based on model simulation show that damping can be controlled by adjusting operating parameters through means such as generation re-dispatch, or load reduction as a last resort. Damping ratios decrease consistently with the increase of overall system stress levels. At the same stress level (i.e., same total system load), inter-area oscillation modes can be controlled by adjusting power flow patterns to reduce flow on the interconnecting tie line(s). The effectiveness of the MANGO control is dependent on specific locations where the adjustment is applied.

The MANGO procedure consists of three major steps:

1. Recognition - operator recognizes the need for operating point adjustment through online ModeMeter monitoring;

2. Implementation - operator implements the adjustment per recommendations by the MANGO procedure; and

3. Evaluation - operator evaluates the effectiveness of the adjustment using ModeMeter and repeats the procedure if necessary.

The recognition and evaluation steps in the MANGO procedure rely on a good ModeMeter to estimate the current modes, while the implementation step builds on modal sensitivity, i.e., the relationship of oscillation modes and operating parameters. The relationship is generally nonlinear, and it is impractical to derive a closed-form analytical solution for this relationship. Theoretically, the sensitivity can be calculated from the model of a power system, but the model is usually not able to reflect real-time operating conditions. Therefore, the work reported here has been primarily centered on estimating modal sensitivity from real-time measurement. Eigenvalue-theory-based sensitivity has been studied and concluded to be not applicable for real-time estimation. Many items in the eigen-sensitivity equations cannot be estimated with regular phasor measurements. Three approaches for estimating modal sensitivities are then proposed and studied to approximate the sensitivity relationship. These approaches are relative sensitivity estimation, artificial neural network (ANN)-based nonlinear mapping, and decision-tree-based rule derivation. The relative sensitivity is formulated using least squares principles in the form that can be estimated directly from measurement. Testing has been carried out with a mediumsized 34-machine system model, analogous to the western interconnection, or Western Electricity Coordinating Council (WECC) reliability region. The results indicate how strong generation output relates to the damping, giving clear guidance which generator needs to be adjusted to improve damping. ANN-based nonlinear mapping has been studied, and it can successfully approximate the modal 
sensitivity from testing results with medium-size systems. Also, a decision-tree-based method is studied to summarize the relationship between operator controllable variables and modes.

System topology changes the inherent dynamics in a power system, and thus changes the modal properties. Topology analysis is conducted to characterize the impact of topology change on oscillation modes. Within the WECC system, topology changes that just modify the impedance along an energy path have significantly more impact on mode damping than on mode frequency. However, there are some topology changes that completely eliminate an important path, and sometimes an entire mode. Even when the mode is not eliminated, it may reappear at a quite different frequency, though with a similar mode shape. Including topology information in the MANGO procedure is very important to its successful application. Topology impacts the MANGO procedure in two aspects: as input, it may significantly change the sensitivity relationship between operation parameters and mode damping, and as output, topology adjustment can be another option for damping control, as shown in the results with the 34-machine model. This merits further study.

In summary, significant progress has been made. A MANGO procedure has been established with practical considerations. The key step in the procedure is the modal sensitivity. Three approaches - direct modal sensitivity estimation, ANN-based nonlinear mapping, and a decision-tree method - have been formulated and studied with promising results from a medium-size system. Impact of topology change on damping has been studied. The simulation studies were conducted with commercialized software, and the resulting experience and data pave the road for large-scale MANGO application. Further work will continue the development of modal sensitivity estimation and application, specifically including work as follows:

1. Enhance the understanding of the relationship between operating points and mode damping for a practical large system (e.g., the WECC system);

2. Enhance the understanding of the influence of topology changes on major modes;

3. Test and refine the MANGO methods with a larger model;

4. Study the impact of mode meter accuracy on modal sensitivity estimation; and

5. Identify potential implementation strategies with operating procedures.

The WECC Wide Area Measurement System (WAMS) and the Eastern Interconnection phasor network are the basis for the proposed scope. The Bonneville Power Administration (BPA) will continue to support relevant WECC WAMS activities, including mode meter development and WECC probing tests. Primary partners are major utility companies (e.g., BPA and the California Independent System Operator (CAISO)). The California Energy Commission (CEC) supports PNNL for a 2-year project in the implementation and deployment of the MANGO techniques developed under the DOE funded effort. This sets up a good path forward for technology transfer. 


\section{Acknowledgement}

The authors would like to thank Mr. Phil Overholt with the U.S. Department of Energy, Dr. Merwin Brown, Mr. Jim Cole and Mr. Larry Miller with California Institute of Energy and Environment, Dr. Dmitry Kosterev with the Bonneville Power Administration, Mr. Hani Alarian and Mr. Matthew Varghese with the California Independent System Operator, Mr. Soumen Ghosh formerly with the California Independent System Operator, Dr. John Pierre with the University of Wyoming, and Dr. Manu Parashar formerly with the Electric Power Group, for their support and productive discussions. 


\section{Acronyms}

2A4M Two-Area, 4-Machine Model

ANN Artificial Neural Network

BPA Bonneville Power Administration

CAISO California Independent System Operator

CERTS Consortium for Electric Reliability Solutions

CEC California Energy Commission

DR Damping Ratio

DSA Dynamic Security Assessment

DSI Dynamic System Identification

DSO Dispatcher Standing Order

DT Decision Tree

EMS Energy Management System

FACTS Flexible AC Transmission System

GE General Electric

GUI Graphical User Interface

IDC Interchange Distribution Calculator

IROL Interconnection Reliability Operation Limit

MANGO Modal Analysis for Grid Operations

MIMO Multiple Input, Multiple Output

MSR Modified Stepwise Regression

NERC North American Electric Reliability Corporation

PDCI Pacific DC Intertie

PDT Pacific Daylight Time

PSLF Positive Sequence Load Flow 
PSS Power System Stabilizer

PST Power System Toolbox

R3LS Regularized Robust Recursive Least Squares

RAS Remedial Action Scheme

SMIB Single-Machine, Infinite Bus

SOL System Operation Limit

SPS Special Protection Scheme

SSAT Small Signal Stability Analysis Tool

TLR Tie Line Relief

TSAT Transient Stability Analysis Tool

WECC Western Electricity Coordinating Council 


\section{Contents}

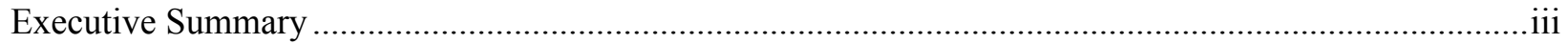

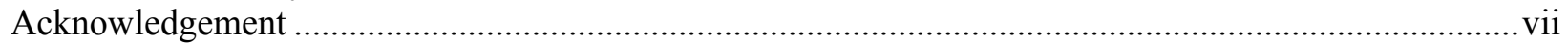

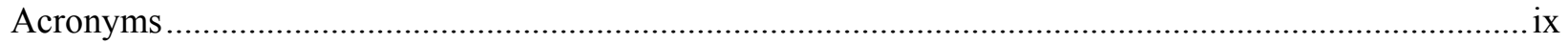

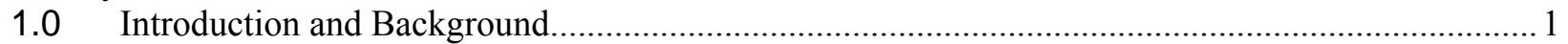

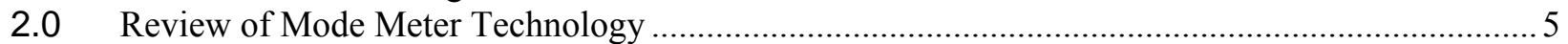

3.0 Relationship of Modal Damping and Operating Conditions....................................................... 11

3.1 Single-Machine-Infinite-Bus System............................................................................. 11

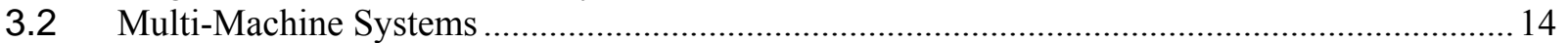

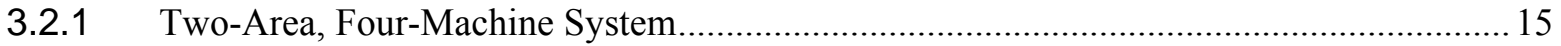

3.2.2 17-Machine System............................................................................................. 17

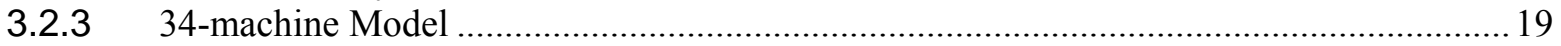

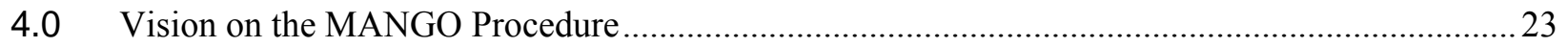

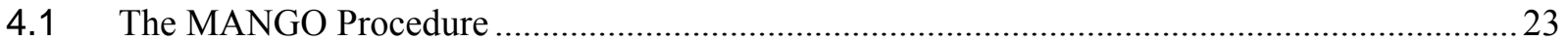

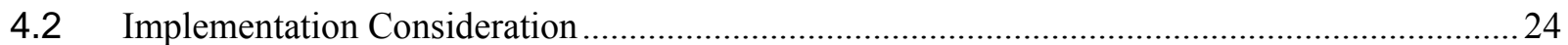

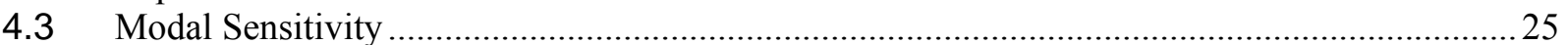

5.0 MANGO Method 1: Relative Modal Sensitivity ……………………………………………...2

5.1 Modal Sensitivity Derived from Eigenvalue Theory ..........................................................2

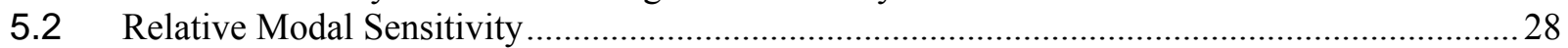

5.2.1 Relative Sensitivity Estimation from Real-Time Measurement ……………………….......30

5.2.2 Case Studies Using the Two-Area, Four-Machine System.................................................... 31

5.2.3 Case Studies Using the 34-machine Model …………………………………………........ 33

5.3 Relative Modal Sensitivity Estimation with respect to Tie Line Flows.........................................35

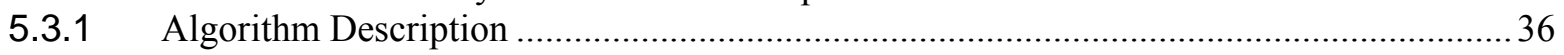

5.3.2 Case Studies Using a Two-Area Four-Machine System ...................................................... 38

5.3.3 Case Studies Using the 34-machine Model ……………................................................ 41

6.0 MANGO Method 2: Nonlinear Mapping Based on Artificial Neural Network.................................45

7.0 MANGO Method 3: Operation Procedure Derivation from Decision Tree......................................49

7.1 Decision-Tree-Based Method for Improving Damping of Inter-area Oscillations .......................49

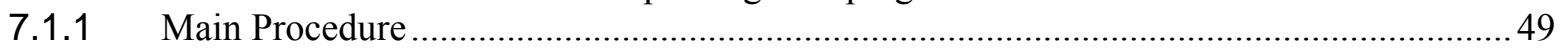

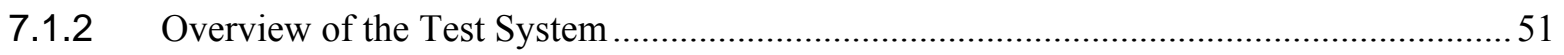

7.1.3 Selection of Critical Generators Using Modal Sensitivity ...................................................5 53

7.1.4 Operational Procedure Using the Decision-Tree Method .....................................................5 54

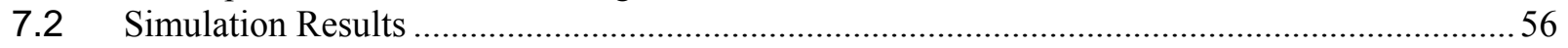

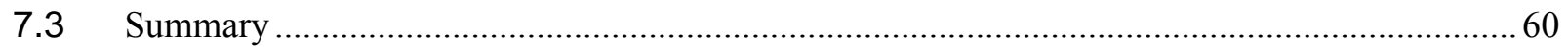

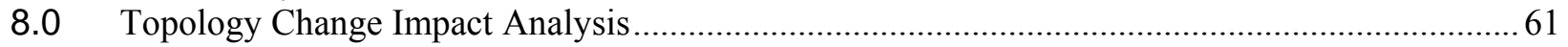

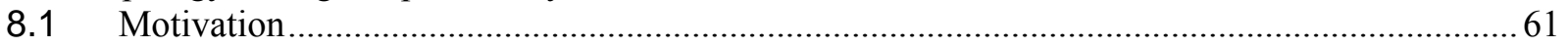

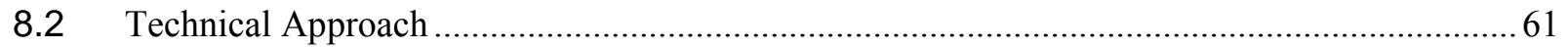

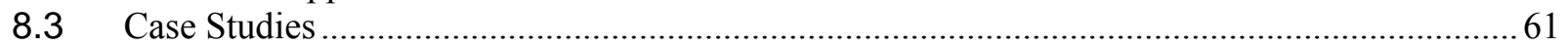

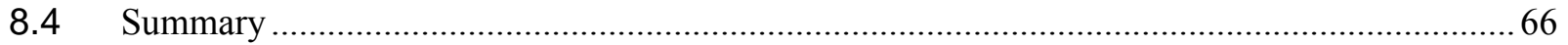

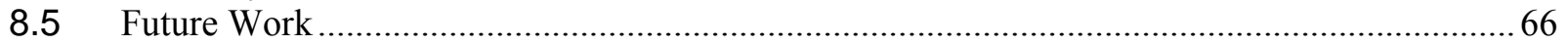

9.0 Experience with Simulation Tools for Studying MANGO ……………………………………....6

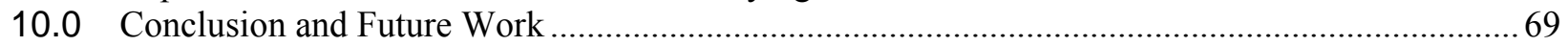

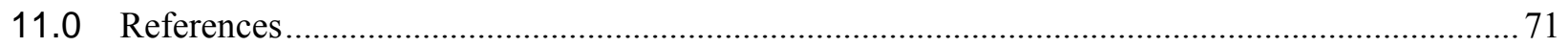




\section{Figures}

Figure 1. Undamped oscillations of the August 10, 1996 western system breakup event ....................... 1

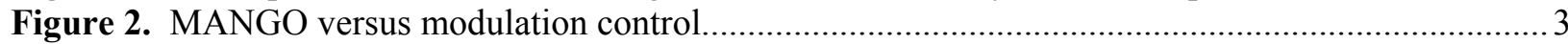

Figure 3. Observed and simulated California-Oregon Intertie power flow during McNary Generator

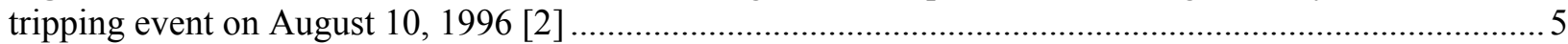

Figure 4. Measurement-based mode analysis on the breakup data of August 10, 1996 ....................... 6

Figure 5. PNNL ModeMeter Prototype for 1996 data playback ........................................................ 7

Figure 6. Mode frequency and damping ratio estimation using R3LS algorithm ............................... 8

Figure 7. Recorded real power flow from Malin to Round Mountain with detected oscillation (Reference

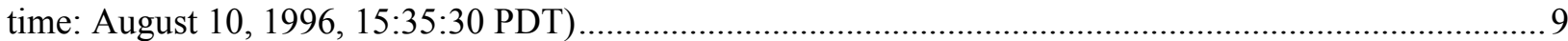

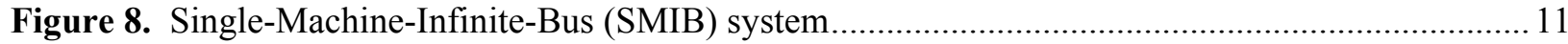

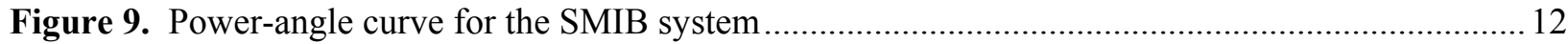

Figure 10. Frequency and damping ratio versus generator power output ............................................. 13

Figure 11. Correlation between the damping ratio and generator power in the SMIB system ................ 14

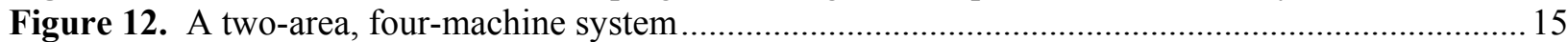

Figure 13. Correlation of the damping ratio with the system stress level (2A4M System) .................... 16

Figure 14. Correlation of the damping ratio with the tie line flow in response to generation re-dispatch of

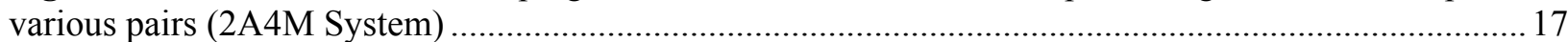

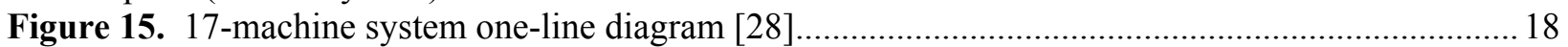

Figure 16. Correlation of damping with the tie line flow of Bus 37 - Bus 20 in the 17-machine system. 19

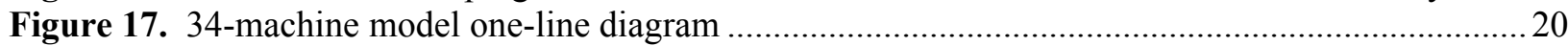

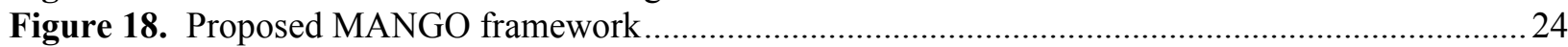

Figure 19. Modal sensitivity of the $0.4-\mathrm{Hz}$ mode in the 17 -machine system.......................................2. 28

Figure 20. Relative modal sensitivity of the $0.4 \mathrm{~Hz}$ mode in the 17 -machine system ............................30

Figure 21. Estimated modal sensitivity of the two-area, four-machine system ................................... 32

Figure 22. Effectiveness of MANGO recommended adjustments for two-area, four-machine system.... 33

Figure 23. Estimated modal sensitivity of the 34-machine model .......................................................34

Figure 24. Effectiveness of MANGO recommended adjustment for 34-machine model ........................35

Figure 25. Effectiveness of MANGO recommended adjustment for the 34 machine model ..................35

Figure 26. Percentage of Contributions from line flow to the damping ratio in the two-area, four-machine

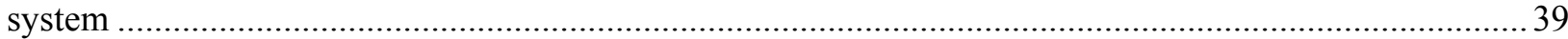

Figure 27. Transmission line with major modal sensitivities in the two-area, four-machine system [42] 40

Figure 28. Percentage of contributions from line flow to the damping ratio in the 34-machine model ... 43

Figure 29. Transmission lines with major modal sensitivities in the 34-machine model .......................44

Figure 30. Training and testing results of the ANN for the $0.4 \mathrm{~Hz}$ mode ..........................................46

Figure 31. MATLAB GUI for testing the proposed ANN-based MANGO approach ...........................46

Figure 32. MANGO control for improving the Alberta mode damping ................................................4 47

Figure 33. Geographical distribution of top five MANGO controls........................................................ 48

Figure 34. Decision-tree-based method to improve damping of inter-area oscillations .........................50

Figure 35. Single-line diagram of 179-bus simplified WECC system ................................................52

Figure 36. Mode shape of the $0.3759-\mathrm{Hz}$ oscillation in the simplified 179-bus WECC system...............53

Figure 37. Sensitivity of the DR of the $0.3759 \mathrm{~Hz}$ mode with respect to generator output .....................54

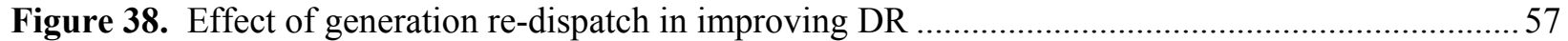

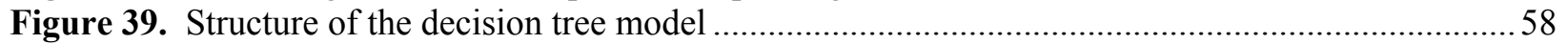

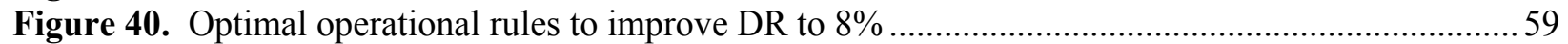

Figure 41. Damping ratio and frequency of all modes in the base case simulation. The blue box

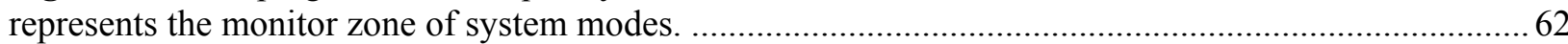

Figure 42. All modes identified in the monitor windows of all topological study cases ........................63 
Figure 43. More detailed view of all modes identified in the monitor windows of all topological study

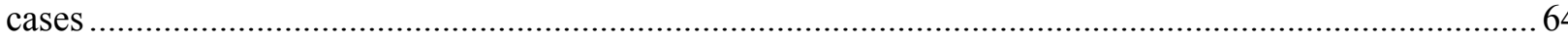

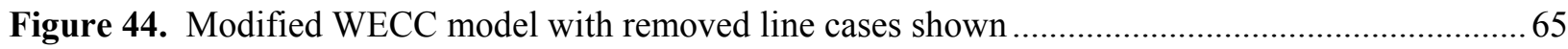

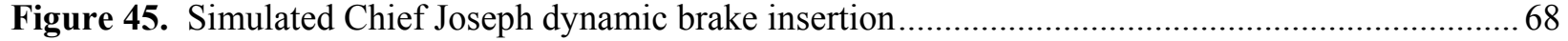




\section{Tables}

Table 1. Base Modal Properties of Two-Area, Four-Machine Model.................................................. 15

Table 2. Selected Modal Properties of 17-machine Model ................................................................... 17

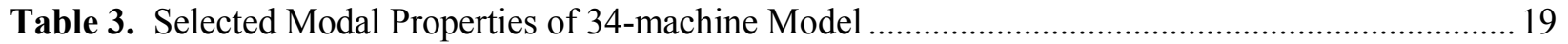

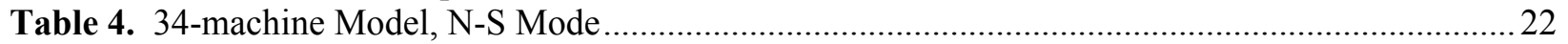

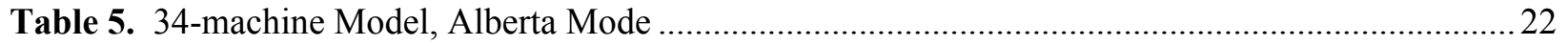

Table 6. Mode Sensitivity with Respect to Line Flow in the Two-Area, Four-Machine System ............. 39

Table 7. Validation of Mode Sensitivities with Respect to Line Flow in the Two-Area, Four-Machine

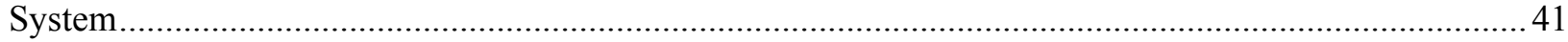

Table 8. Modal Sensitivity with Respect to Line Flow for the 34-machine Model ................................ 42

Table 9. Selected Modal Properties of 179-bus System Model …........................................................51

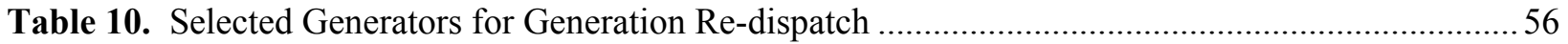

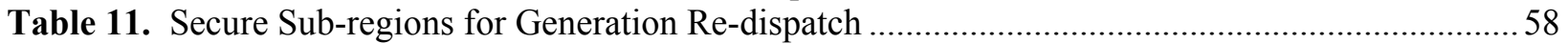




\subsection{Introduction and Background}

Small signal stability problems have long been recognized as one of the major threats to power grid stability and reliability. An undamped mode can cause large-amplitude oscillations and may result in system breakup and large-scale blackouts [1]. There have been several incidents of system-wide lowfrequency oscillations. Of them, the most notable is the August 10, 1996 western North American power system breakup involving undamped system-wide oscillations [2]. In the August 10, 1996 event, the system deteriorated over time after the first line was tripped around 15:42 (Figure 1). About 6 minutes later, undamped oscillations occurred and the system broke up into several islands. Other oscillation events in the U.S. and elsewhere have been observed [3][4]. They all exhibited sustained low-frequency oscillations and have led to a great concern about the adverse effect of oscillations on power system operation.

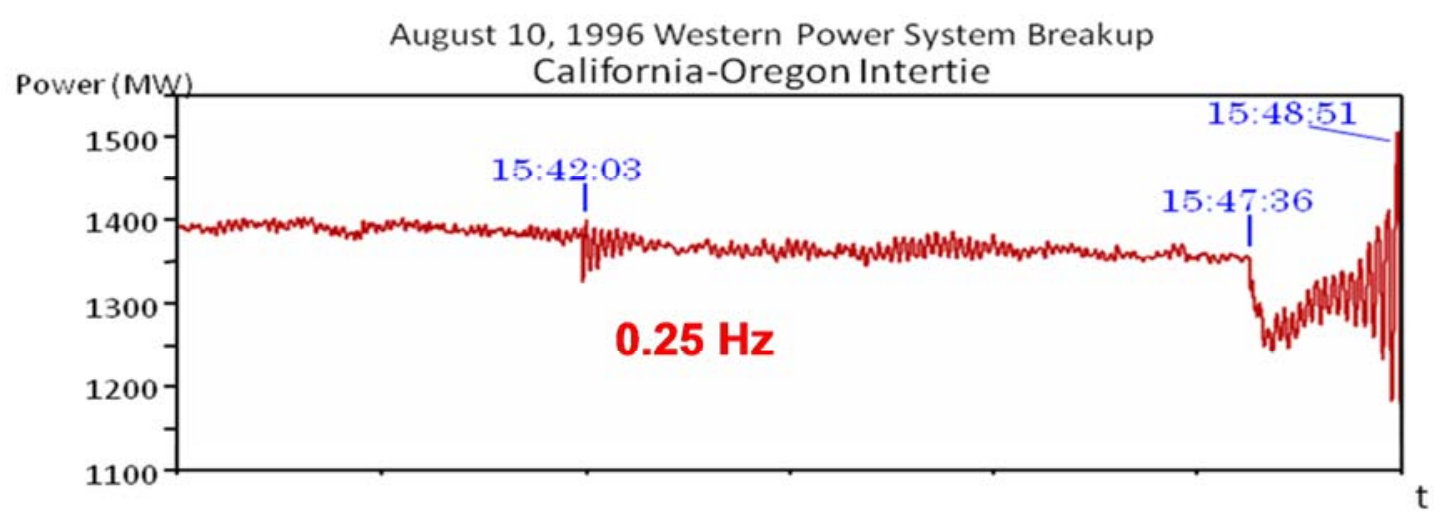

Figure 1. Undamped oscillations of the August 10, 1996 western system breakup event

It is natural to categorize power system oscillations according to the operational setting in which they occur, and according to the types of equipment involved. Operationally, three kinds of major oscillations have been encountered:

- Spontaneous oscillations, occurring under ambient system conditions. These usually grow slowly, from initially low levels.

- Transient oscillations, triggered by loss of a major generation, load, or delivery resource. These tend to be large at the onset, and poorly damped if the post-disturbance network has an undamped mode.

- Forced oscillations, once a common result of delayed tripping for lines between asynchronous islands. These tended to be large at the onset, and to persist until islanding was completed. This has become rare, and forced oscillations would usually reflect some kind of testing or control problems.

Different oscillation problems require different countermeasures. A controller designed to provide damping under ambient conditions (an ambient damper) will usually respond to oscillations of all three types, for lack of contrary information. Its tuning may be quite inappropriate for transient oscillations, or even for step disturbances. A transient damper would usually ignore ambient oscillations and use step 
disturbances as arming information. Local information would probably not permit it to distinguish between transient and forced oscillations, however. The dynamic effects of either an ambient damper or a transient damper during forced oscillations may well be harmful, especially if it is a high performance device with self-tuning capabilities.

Reference [1] is a useful introduction to the extensive literature on this subject. The following general guidelines can be extracted from this and from Western Electricity Coordinating Council (WECC) experience:

- Some degree of oscillatory activity is normal. To recognize abnormal behavior, one must know the limits of normal behavior.

- Abnormal oscillations are usually a symptom of some deeper problems. Special damping controls may provide a temporary remedy, but ultimately the underlying deficiency must be addressed. In real-time operation, this usually involves power flow adjustments.

- The ultimate and essential defense against widespread oscillations is to cut the interaction paths. The necessary actions range from tripping a troublesome generator to controlled separation of the system into stable islands.

- Major disturbances - and transient oscillations - usually result from a major change in power system topology. Local control schemes may not be set for this-Wide-area control requires wide-area information.

The general thrust of utility experience with system upsets [5][6] argues that automated wide-area controls are necessary, but not in themselves sufficient to deal with the wide range of challenges that confront emergency management in a large power system. The essential ingredient is actionable information at the operator level, consisting of:

- Situational awareness, observation of system conditions related to a knowledge base regarding their implications; and

- Operational resources, countermeasure options plus the authority and facilities to execute them.

The arming of special stability controls is a well recognized element among operational resources necessary to the western interconnection. The major challenge is to develop a knowledge base indicating the conditions under which these and more complex countermeasures are appropriate. This is a major objective of this project, aiming to develop modal analysis for grid operations (MANGO).

In power systems, low-frequency oscillations are a result of electromechanical coupling between the transmission network and generators. Considerable understanding and literature have been developed over the past several decades of the cases where these oscillations become very lightly damped, or even unstable. Small-signal stability studies have been mainly based on the eigenvalue analysis of the characteristic matrix derived from the linearized model of a power system [7]. Power system stabilizers (PSS) have been used for damping control in common industrial practice. While effective in damping oscillations, especially local-mode oscillations, PSS tuning is a very challenging task for inter-area oscillation modes because of time-varying operating conditions. The practical feasibility of PSS for inter- 
area modes is further limited because power system models have been found inadequate in describing real-time operating conditions [2][8].

A desirable alternative to PSS is to bring the system to a new operating condition through operator actions or automated actions if appropriate. The new operating condition would have damping high enough to sustain disturbances so oscillations would not occur. This project aims to develop such an operationoriented approach to perform MANGO analysis and provide operation recommendations. Because small signal stability is an inherent characteristic of a dynamic system, the low damping situation can be detected from ambient measurements before a disturbance triggers the oscillation [9], or from disturbance data as soon as the disturbance changes the modal properties. ModeMeter technology has been demonstrated to have the real-time capability of estimating system modes from both oscillation signals and ambient data [10]. The MANGO control can be applied as a preventive or corrective measure to improve damping and prevent oscillations once low damping situation is detected.

Different from PSS and other modulation-based methods, MANGO aims to improve damping through adjusting operating points. Traditionally, the modulation-based methods do not change the system's operating point, but improve damping through automatic feedback control [11][12][13]. Figure 2 illustrates the difference of these two types of damping improvement methods. Availability of wide-area phasor measurements enables wide-area modulation control for control devices such as the Pacific DC Intertie (PDCI) in the western US power system and Flexible AC Transmission Systems (FACTS) devices [12]. MANGO, shown in red, and modulation control, shown in magenta, are complementary towards the same objective. Modulation-based methods are designed to maintain positive damping at expected operating conditions, whereas MANGO-recommended adjustments can be used to move the system to a more stable operating point that damping would be sufficient in the event of a system disturbance.

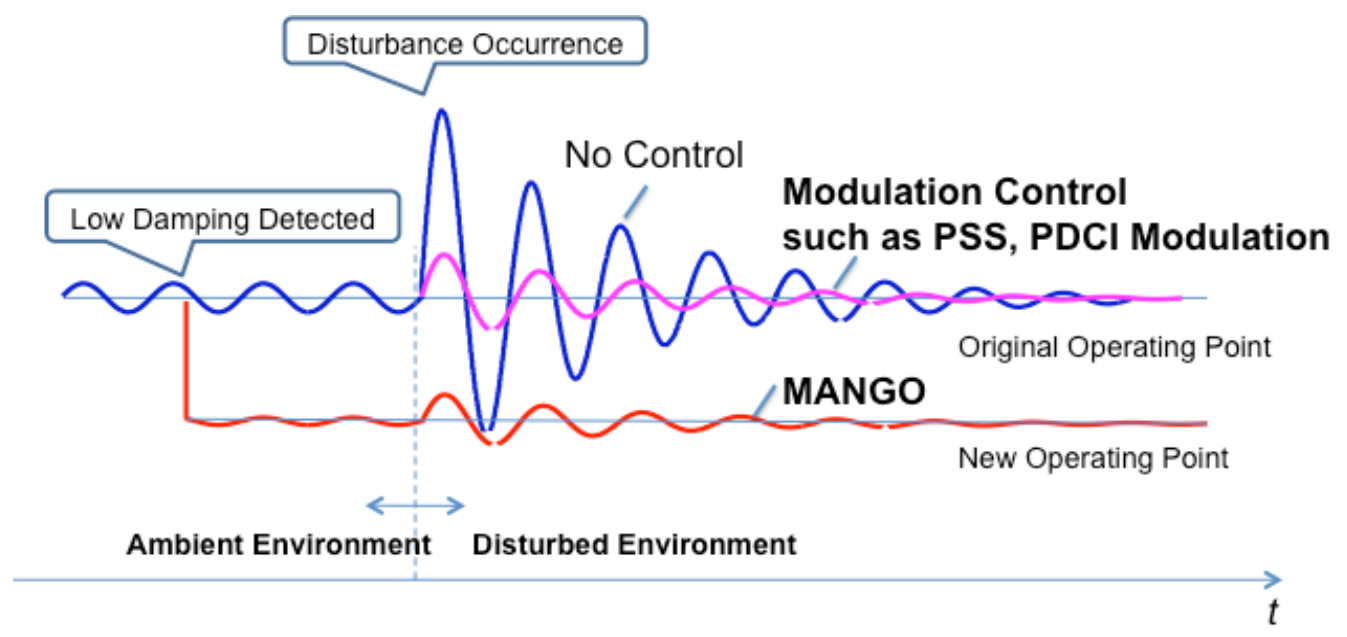

Figure 2. MANGO versus modulation control

Phasor measurements becoming widely available and ModeMeter techniques maturing provide a solid foundation for the development of MANGO technology. MANGO is expected to bring modal information from a monitoring tool to a decision support tool and bridge the gap between modal analysis and power grid operations. To achieve this goal, a key step in MANGO is to identify modal sensitivity with respect to controllable operating parameters, such as generation and load. 
This report reviews the ModeMeter techniques (Section 2), explores the fundamental relationship between modal damping and operating parameters (Section 3), and examines the vision of the MANGO project (Section 4). The report continues to propose the concept of relative modal sensitivity and how it can be estimated and may be used in damping control (Section 5). Section 6 discusses an artificial neural network approach applied to the MANGO data. Section 7 explores a decision-tree approach for MANGO, and Section 8 explores the effect of topology changes on modal properties in a power system. Section 9 discusses some of the different programs and data experience from the MANGO project, and the report concludes in Section 10. 


\subsection{Review of Mode Meter Technology}

Modes are the eigenvalues of a linearized power system models [7]. Generally, there are two basic approaches for estimating power system modes: component-based methods and measurement-based methods. With the component-based method, the nonlinear differential equations governing the system are linearized around an operating point. The power system modes are then obtained through eigenvalue analysis [14]. On the other hand, for a measurement-based method, a linear model is estimated from direct system measurements [15].

An important aspect to remember is that for a large complex power system, the efforts of building a component-based model are not trivial. For example, an initial effort was made by Kosterev et al. [2] to build a component-based model for simulating the Western Electricity Coordinating Council (WECC) reaction during August 10, 1996 system breakup event. The simulation result from the initial model did not match the field measurement data (Figure 3). Matched simulation and measurement results were only achieved after extensive studies, months later. In contrast, a measurement-based approach usually requires significantly less effort. The measurement-based method can update the mode estimation based on incoming measurement data. Thus, the measurement-based methods can serve as a good complement to model-based methods in monitoring power system modes in real time.

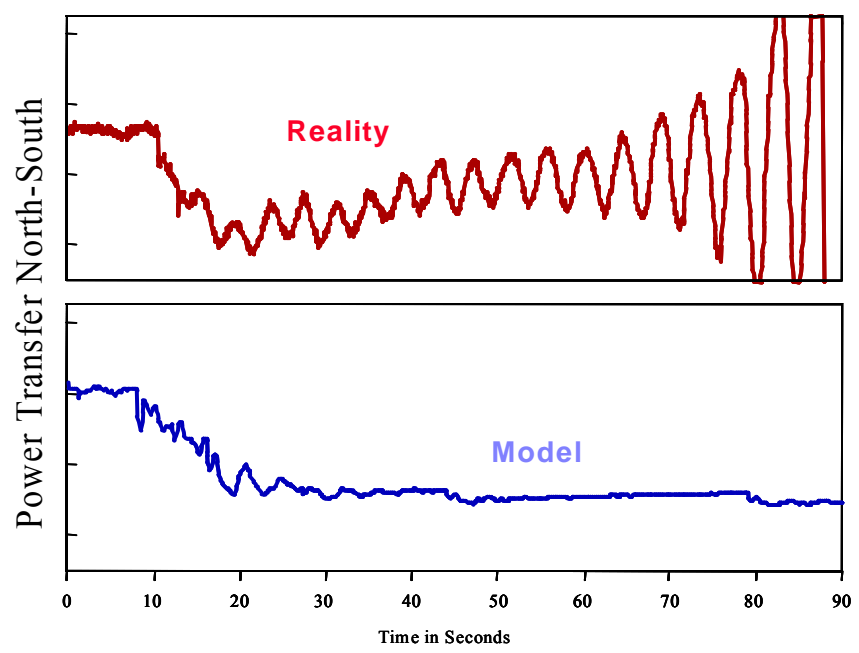

Figure 3. Observed and simulated California-Oregon Intertie power flow during McNary Generator tripping event on August 10, 1996 [2]

For a measurement-based method, a general linear model structure is first selected, and then model parameters are identified to fit the measurements. Mode estimation methods based on measurement data have been extensively studied. A sample of papers includes [15], [16], [17], [18], [19], [20], [21], [22], and [23]. Measurement-based models usually take much less effort to build than those required for a model-based method. Some initial studies were carried out by Drs. John Hauer and John Pierre on the western interconnection breakup on August 10, 1996 using measurement-based methods. The results are summarized in Figure 4 below. It can be observed that the $0.25-\mathrm{Hz}$ oscillation, which was indicative of the impending breakup, is identified. The results also show that the oscillation frequency decreases from $0.27 \mathrm{~Hz}$ to $0.25 \mathrm{~Hz}$, and the oscillation damping ratio decreases from $7.0 \%$ to $1.2 \%$. These are clear indications of the approaching small signal stability problem. This study shows the great potential of measurement-based methods in monitoring power system oscillation modes. 


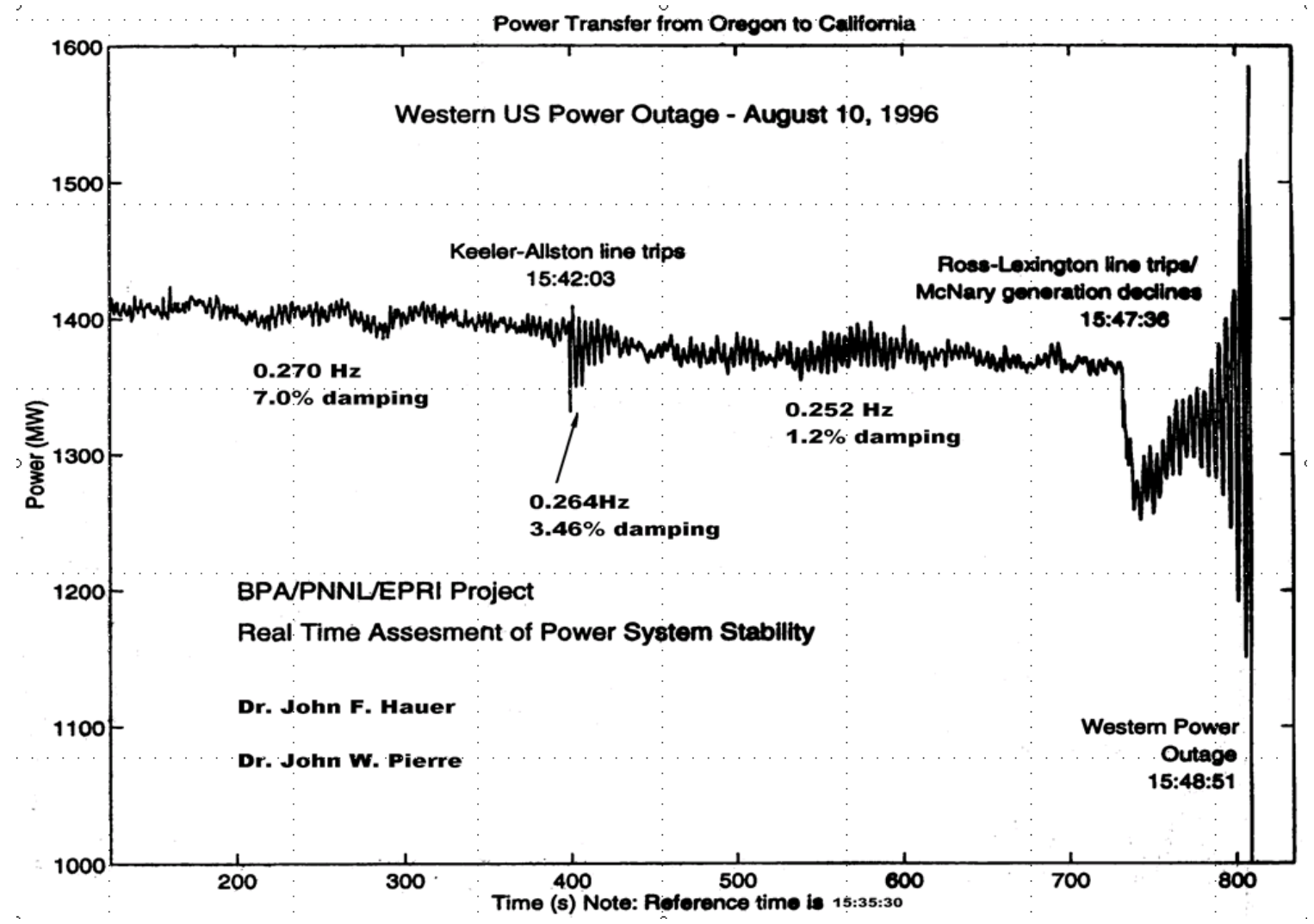

Figure 4. Measurement-based mode analysis on the breakup data of August 10, 1996

As discussed by Hauer et al. [10], any measurement-based approach that automatically estimates modes in near real time has significant potential for system operation and control applications. Such algorithms are termed "ModeMeter" algorithms [10]. A "ModeMeter" tool developed by PNNL based on a regularized robust recursive least square (R3LS) algorithm is shown in Figure 5 [9][18][24]. The prototype ModeMeter tool has been validated using a 17-machine model and tested with field measurement data from the WECC. 


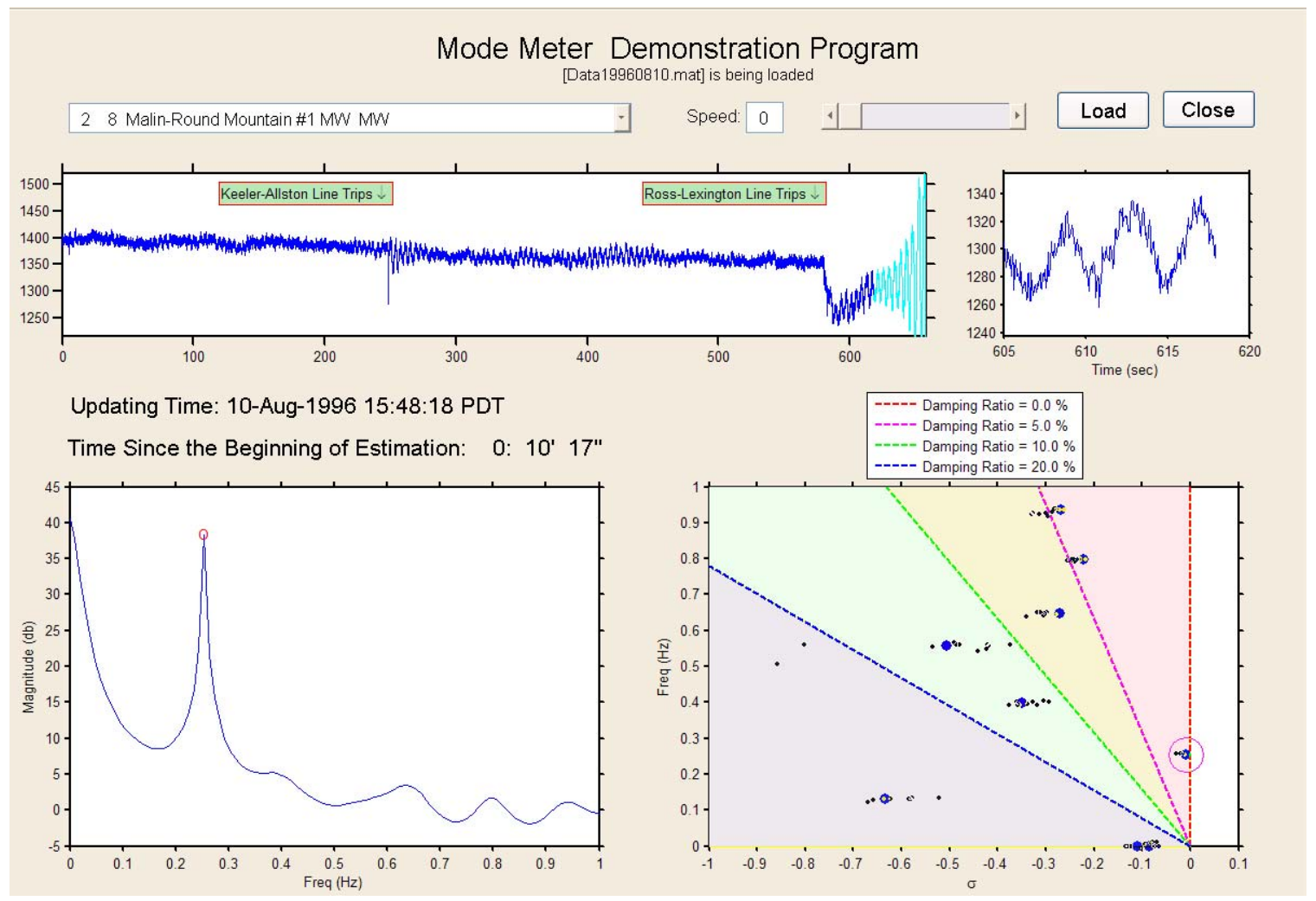

Figure 5. PNNL ModeMeter Prototype for 1996 data playback

In the recent study [25], it is revealed that performance of the mode estimation can be improved when a proper mode identification algorithm can be applied to the right type of measurement data. In general, field measurement data can be classified into two categories: typical and non-typical data. Typical data is the data that carries system mode information and can be described by the model structure used by an identification algorithm. In contrast, non-typical data does not carry information about system modes and cannot be described by a general linear model.

Commonly encountered non-typical data points include, but are not limited to, missing data and outliers. Missing data are often dropped data points, which may result from temporary communication and measurement device failure. Outliers are values that significantly deviate from normal values. Outliers may result from a serious disturbance and/or sensor failure. In general, data that cannot be described by the adopted model structure is considered non-typical data. For example, transient data right before ringdown signals is also considered non-typical, namely because it cannot be described by a linear prediction model.

Commonly encountered typical data can include, but are not limited to, ambient data, ringdown oscillations, and probing data. Ambient data is obtained when a system is working under an equilibrium condition, and the major disturbance is from small-amplitude random load changes. Ringdown oscillation data occurs after some major disturbance, such as a line tripping, and results in observable oscillations. Probing data is obtained when low-level pseudo-random noise is intentionally injected into the system to test the system performance.

Note that these three types of data carry different levels of mode information density. The ringdown oscillation data carries the highest level of information density. The mode estimation converges fast to the 
true values. As such, it is valuable to identify oscillation data from other signals. An oscillation detection and analysis method is reported in [25]. It is shown that an identified ringdown oscillation can help: select right algorithm, reduce the mode estimation time, and provide an indication of the disturbance events.

The mode estimation and oscillation detection results using 1996 breakup data are summarized in Figure 6 and Figure 7 [25][26]. In Figure 7, the major oscillations are detected and marked out. In Figure 6, the blue lines are for the mode estimation from oscillation detection and the recursive Prony analysis method using a 30-second analysis window. The red lines indicate mode estimation with mode initialization using the R3LS method with a 5-minute analysis window. For comparison, the mode estimation results from Figure 4 by Drs. Hauer and Pierre are also shown in the Figure 6 as green dots. Figure 6 also shows the capability of the ModeMeter tool for on-line continuous monitoring of power system oscillation modes. Also observe that the modes can be tracked faster with a short time window when oscillations are detected. The online ModeMeter tool and oscillation detection method have consistent estimation results with the offline expert analysis of Figure 4. Such a ModeMeter tool would have recognized the oscillation and issued an earlier warning of the impending breakup of August 10, 1996. The observations in Figure 4 and Figure 6 also indicate that ModeMeter is becoming mature and ready for real-time applications. This lays a great foundation for MANGO studies.
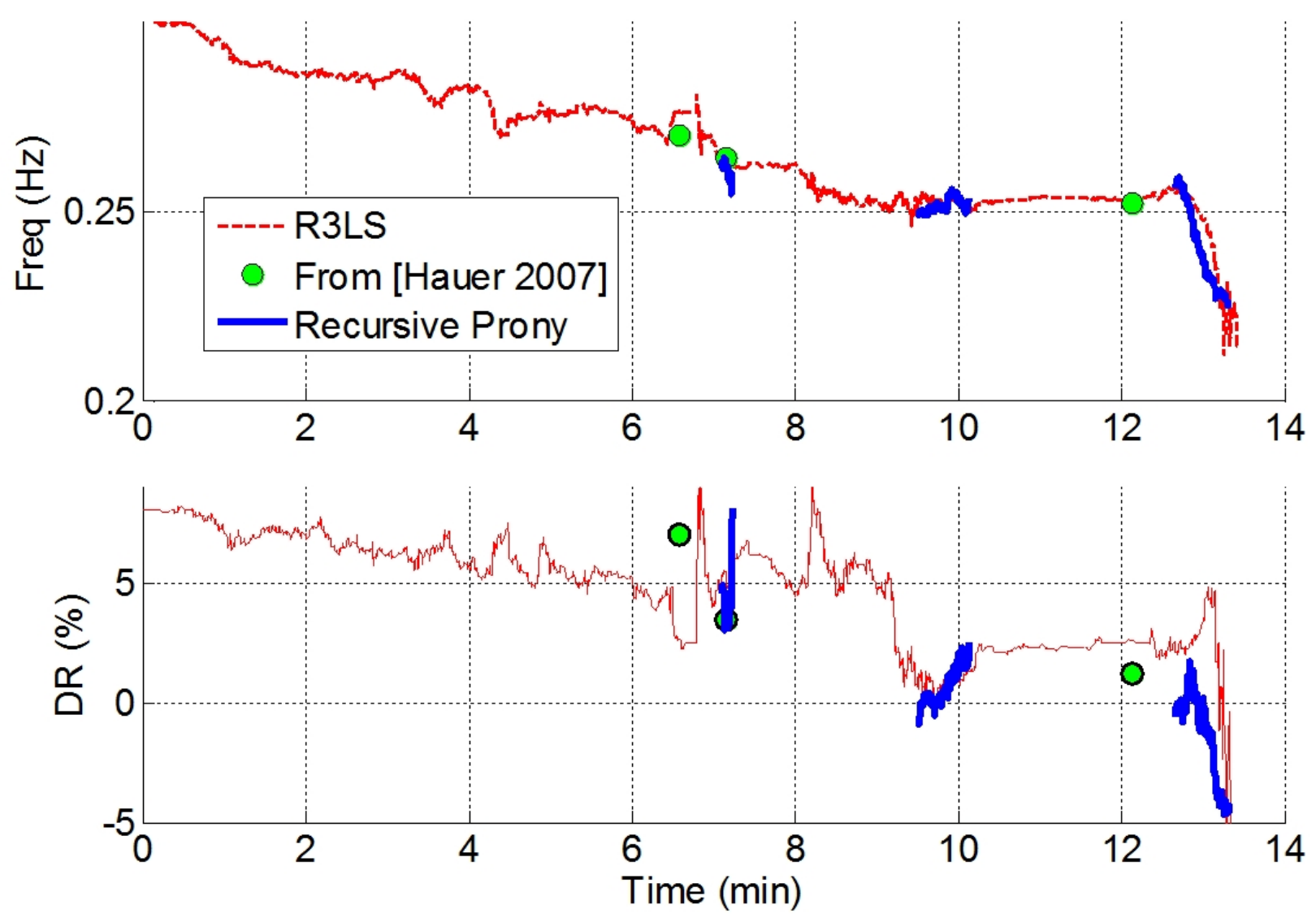

Figure 6. Mode frequency and damping ratio estimation using R3LS algorithm 


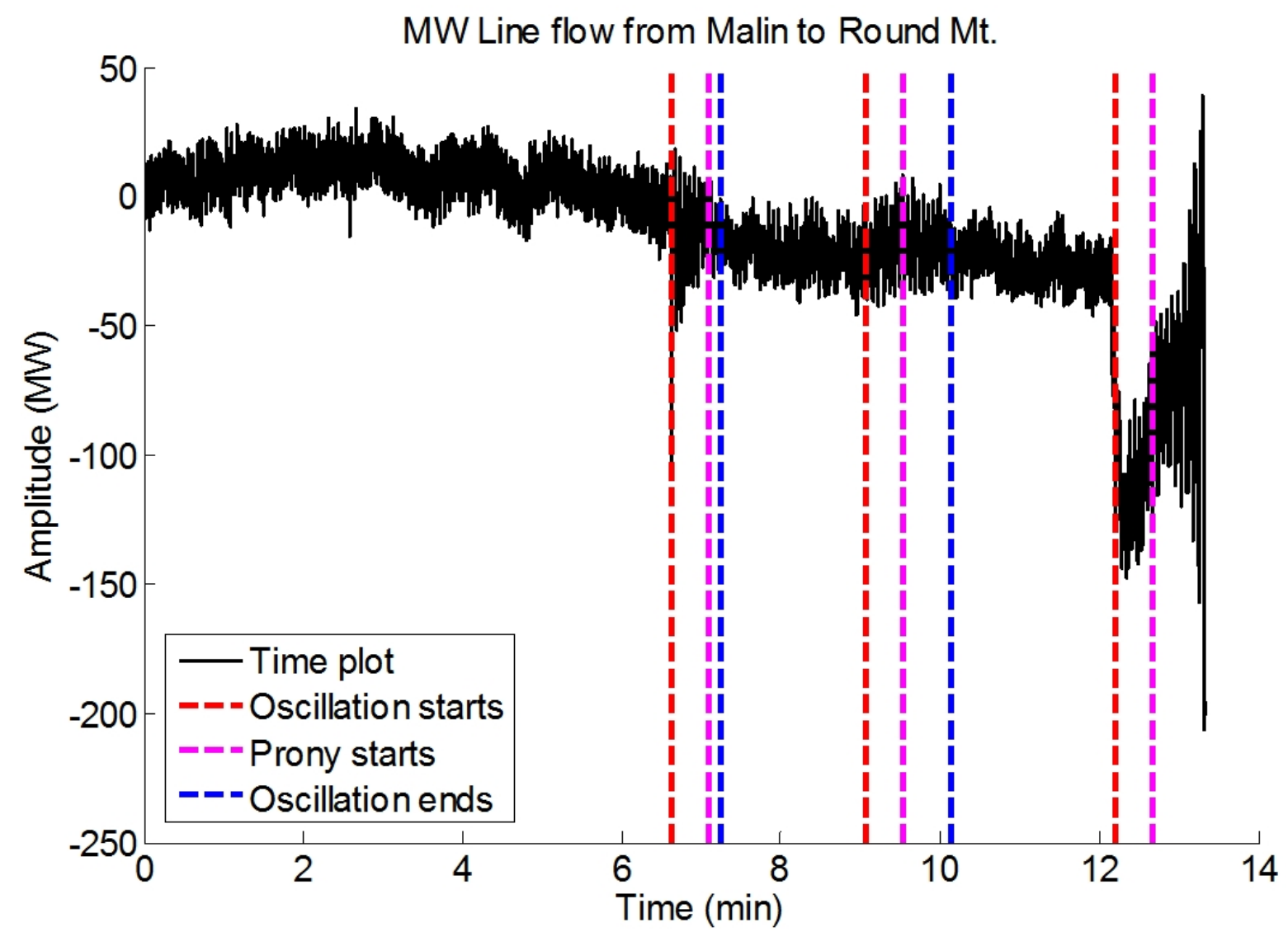

Figure 7. Recorded real power flow from Malin to Round Mountain with detected oscillation (Reference time: August 10, 1996, 15:35:30 PDT) 


\subsection{Relationship of Modal Damping and Operating Conditions}

The operating point of a power system determines the eigenvalues, i.e., the oscillation modes, of the system. A power system can be described as a set of nonlinear differential algebraic equations:

$$
\left\{\begin{array}{l}
\dot{x}=f(x, y, u) \\
0=g(x, y, u)
\end{array}\right.
$$

where $x$ is the state vector, $y$ is the algebraic vector, and $u$ is the input vector. Linearizing these nonlinear equations at an operating point determines the characteristic matrix of the system. The non-real eigenvalues of the characteristic matrix are the oscillation modes of the power system. The characteristic matrix is shown as the A matrix in the following equation:

$$
\left\{\begin{array}{l}
\Delta \dot{x}=\mathbf{A}(p) \Delta x+\mathbf{B}(p) \Delta u \\
\Delta y=\mathbf{C}(p) \Delta x+\mathbf{D}(p) \Delta u
\end{array}\right.
$$

where $p$ represents operating parameters. These parameters may be values such as generator output, load consumption, transformer taps, capacitor MVAr, and DC power settings, which can be adjusted by operators in real-time power system operation. Equation (2) indicates that the A matrix is a function of parameters, $p$. Thus, the modes can be influenced by adjusting some of the $p$ parameters, i.e., changing the operating point of the power system.

\subsection{Single-Machine-Infinite-Bus System}

The Single-Machine-Infinite-Bus (SMIB) system (Figure 8) provides a unique opportunity to observe the effect of operating conditions on small-signal stability because of its simplicity. The primary operating parameter is the steady-state generation output $\mathrm{P}$, which is same as the power transfer on the tie lines to the infinite system. Another operating parameter is the steady-state terminal voltage $V$. These two parameters can be adjusted through reference settings of the governor and the exciter, respectively.

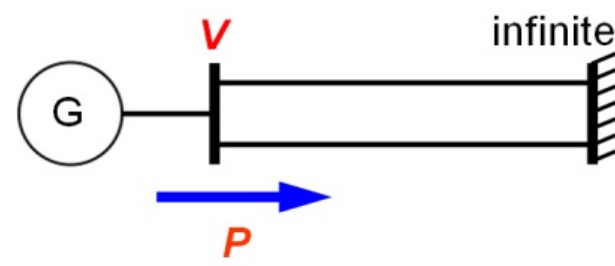

Figure 8. Single-Machine-Infinite-Bus (SMIB) system

Describing this SMIB system with the classical generator model, the following swing equations result: 


$$
\left\{\begin{aligned}
\frac{2 H}{\omega_{s}} \frac{d \omega}{d t} & =T_{m}-T_{e}-D \omega \\
& =\frac{\omega_{s}}{\omega} P_{m}-\frac{\omega_{s}}{\omega} P_{e}-D \omega \\
\frac{d \delta}{d t} & =\omega-\omega_{s}
\end{aligned}\right.
$$

where $\delta$ is the rotor angle in radians, and $\omega$ is speed in radian/second, $T_{m}$ and $T_{e}$ are the mechanical torque and the electrical torque in p.u., $P_{m}$ and $P_{e}$ are the mechanical power input and the electrical power output in p.u., $\omega_{s}$ is the synchronous speed in radian/second, and $H$ and $D$ are the generator inertia constant and damping coefficient, respectively. The tie line power $P\left(=P_{e}\right)$ is a function of the rotor angle $\delta$, the generator internal voltage $E$, infinite bus voltage $V_{\infty}$, and the total impedance $X$ :

$$
P=\frac{E V_{\infty}}{X} \sin \delta
$$

Given constant $E, V_{\infty}$, and $X$, the electrical power follows the power-angle curve shown in Figure 9.

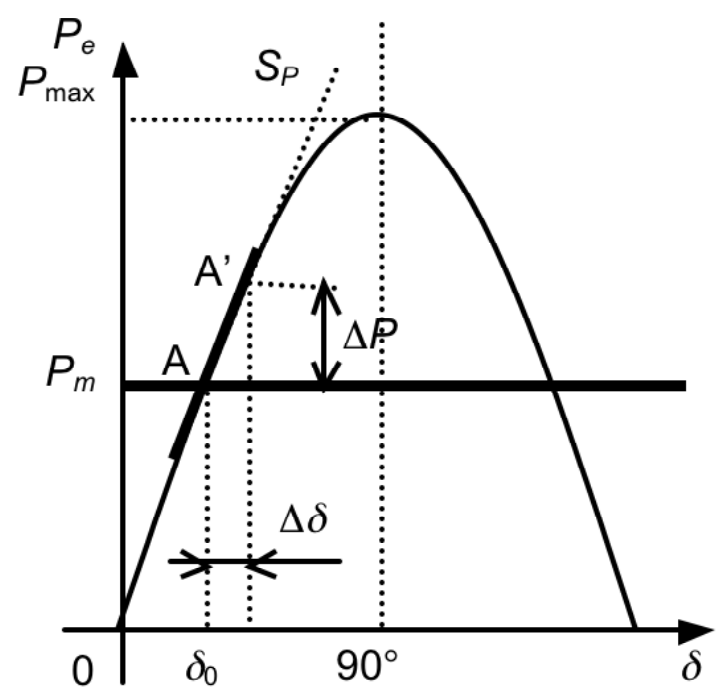

Figure 9. Power-angle curve for the SMIB system

Assuming $T_{m}$ is constant, linearization of Equations (3) and (4) gives the characteristic matrix:

$$
\begin{aligned}
{\left[\begin{array}{c}
\Delta \dot{\omega} \\
\Delta \dot{\delta}
\end{array}\right] } & =\left[\begin{array}{cc}
\frac{1}{2 H} \frac{\omega_{s}^{2}}{\omega_{0}^{2}} P_{e 0}-\frac{\omega_{s}}{2 H} D & -\frac{1}{2 H} \frac{\omega_{s}^{2}}{\omega_{0}} \frac{E V_{\infty}}{X} \cos \delta \\
1
\end{array}\right]\left[\begin{array}{l}
\Delta \omega \\
\Delta \delta
\end{array}\right] \\
& =\left[\begin{array}{cc}
K \frac{\omega_{s}}{\omega_{0}^{2}} P_{e 0}-K D & -K \frac{\omega_{s}}{\omega_{0}} S_{P} \\
1 & 0
\end{array}\right]\left[\begin{array}{l}
\Delta \omega \\
\Delta \delta
\end{array}\right]
\end{aligned}
$$


where $K=\frac{\omega_{S}}{2 H}$, and $S_{P}=P_{\max } \cos \delta_{0} . S_{P}$ is specific to the operating point (Figure 9).

The system oscillation modes, obtained by the eigenvalue analysis of Equation (5), are:

$$
\lambda_{1,2}=-\sigma \pm j \omega=\frac{-\left(-K \frac{\omega_{s}}{\omega_{0}^{2}} P_{e 0}+K D\right) \pm \sqrt{\left(K \frac{\omega_{s}}{\omega_{0}^{2}} P_{e 0}-K D\right)^{2}-4 K \frac{\omega_{s}}{\omega_{0}} S_{P}}}{2}
$$

When the square-root term in Equation (6) is less than zero, the system exhibits oscillatory behaviors, and the damping ratio is:

$$
\xi=\frac{\sigma}{\sqrt{\sigma^{2}+\omega^{2}}}=\frac{-K \frac{\omega_{s}}{\omega_{0}^{2}} P_{e 0}+K D}{\sqrt{4 K \frac{\omega_{s}}{\omega_{0}} S_{P}}}
$$

Figure 10 shows that both the modal frequency and damping decrease as the generator power output increases. Parameters for Figure 10 include $\omega_{0}=377$ radians/second, $D=0, E=1$ p.u., $V_{\infty}=1$ p.u., $\omega_{s}=$ 377 radians/second, $H=3$, and $X=0.1$. This is consistent with Equation (5) and (6). For damping ratio, Equation (7) shows if $P_{e 0}$ increases, $S_{P}$ decreases, and thus the damping ratio decreases. It is interesting to point out that many textbooks assume $w_{0}=w_{s}$, and the effect on the damping is not correctly captured.

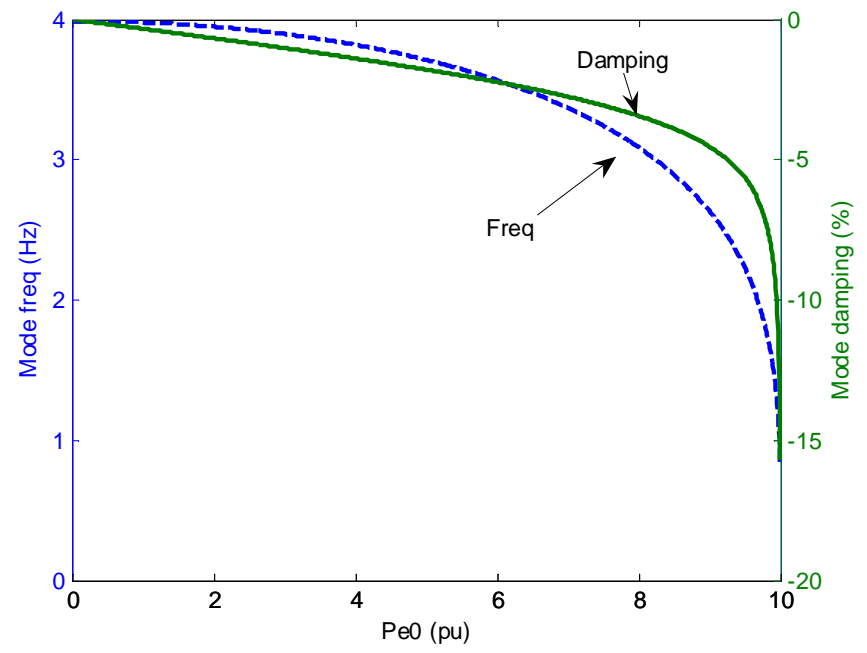

Figure 10. Frequency and damping ratio versus generator power output

To further examine the effect of operating points on the small-signal stability of this SMIB system, expand the generator model to include an exciter and power system stabilizer, randomly vary $P$ and $V$ within their respective operating ranges, and calculate the damping ratio for each operating point. In Figure 11, the damping ratio is shown to have a strong correlation with the generator power output $P$ (or the tie line power). Increasing the power transfer gradually decreases the damping ratio, as indicated by the red arrow line. This is consistent with the finding pointed out in Pai et al. [27] that one of the main reasons for oscillation problems is "the efforts to transmit bulk power over long distance." The small 
deviations from the correlation are caused by the variations in the voltage setting. It clearly shows the effect of the voltage setting is secondary compared to that of the power setting. Adjusting operating points through generation re-dispatch should be an effective means to increase the damping ratio, and thus the small-signal stability.

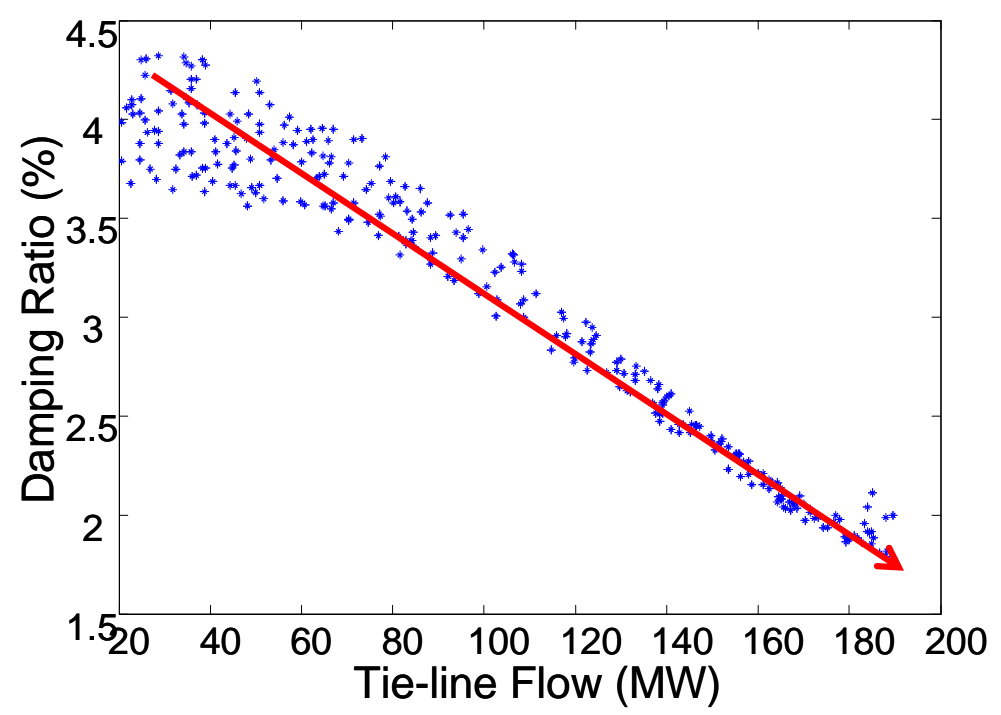

Figure 11. Correlation between the damping ratio and generator power in the SMIB system

\subsection{Multi-Machine Systems}

It is clear that in the SMIB system, damping ratio is affected by the operating point. In such a simple system, the relationship can be viewed as modal sensitivity to the system stress level, generator output, or tie line flow. From the damping control perspective, one may suggest reducing the stress level, generator output, or tie line flow. They are equally effective because they are the same for the SMIB system. However, in real-life power systems, the relationship is much more complicated because there are multiple machines in the system, each having various effects on damping. This section further examines the relationship in multi-machine systems in the following three categories of relationship:

1. Modal damping to the system stress level: The "stress level" is defined as total load and generation in the system. In the studies, the total load and generation are adjusted, but the relative proportion of each load and generation remains unchanged.

2. Modal damping to generation/load pattern: The "generation/load pattern" is defined as the change in relative proportion contributed by each generator/load. Under this category, the total load and generation remain unchanged, so the stress level remains the same, but the percentage contribution of each generator is adjusted to observe the change in oscillation modes.

3. Modal damping to tie line flow: "tie line flow" is adjusted to evaluate its influence on oscillation modes. To account for oscillation problems, operating procedures, such as Bonneville Power Administration (BPA) Dynamic Standing Order 303, have been established to cut tie line flows when lightly damped modes are observed. This study is to investigate how the tie line flow influences an inter-area mode. 
Three multi-machine systems are used for the studies: a two-area-four-machine (2A4M) system [7] (Figure 12), a 17-machine system [28] (Figure 15), and a 34-machine model (Figure 17). All the studies were performed using the Power System Toolbox with MATLAB [14].

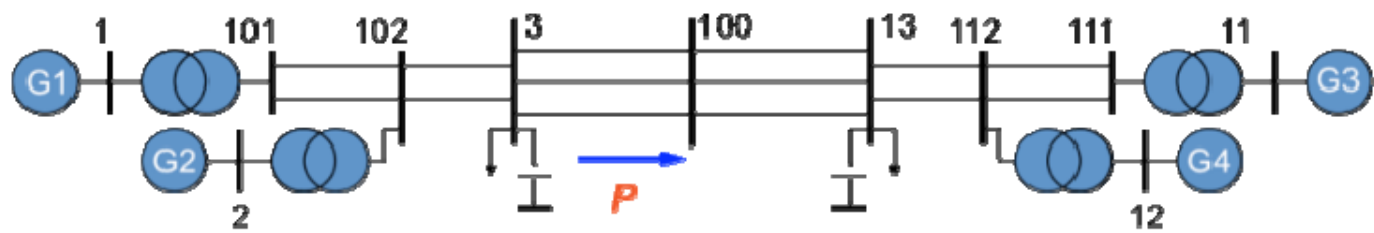

Figure 12. A two-area, four-machine system

\subsubsection{Two-Area, Four-Machine System}

The two-area, four-machine system shown in Figure 12 represents a simple model for small signal stability studies. The model has three primary oscillatory modes of interaction. These modes are shown in Table 1. Of particular interest is the $0.65-\mathrm{Hz}$ interarea mode. This mode represents the interactions of generators 1 and 2 against generators 3 and 4 through the tie line between buses 3 and 13 .

Table 1. Base Modal Properties of Two-Area, Four-Machine Model

\begin{tabular}{|c|c|}
\hline Frequency $(\mathbf{H z})$ & Damping Ratio (\%) \\
\hline 0.6475 & 6.89 \\
\hline 1.09 & 9.50 \\
\hline 1.10 & 9.32 \\
\hline
\end{tabular}

Figure 13 shows the correlation of the damping ratio of the $0.65-\mathrm{Hz}$ mode and the system stress level in the 2A4M system. All the red circles are stressed base cases, which were created by uniformly adjusting load and generation. The blue dots represent disturbed cases. For each stress base case, 100 disturbed cases were generated by randomly adjusting generation patterns. 

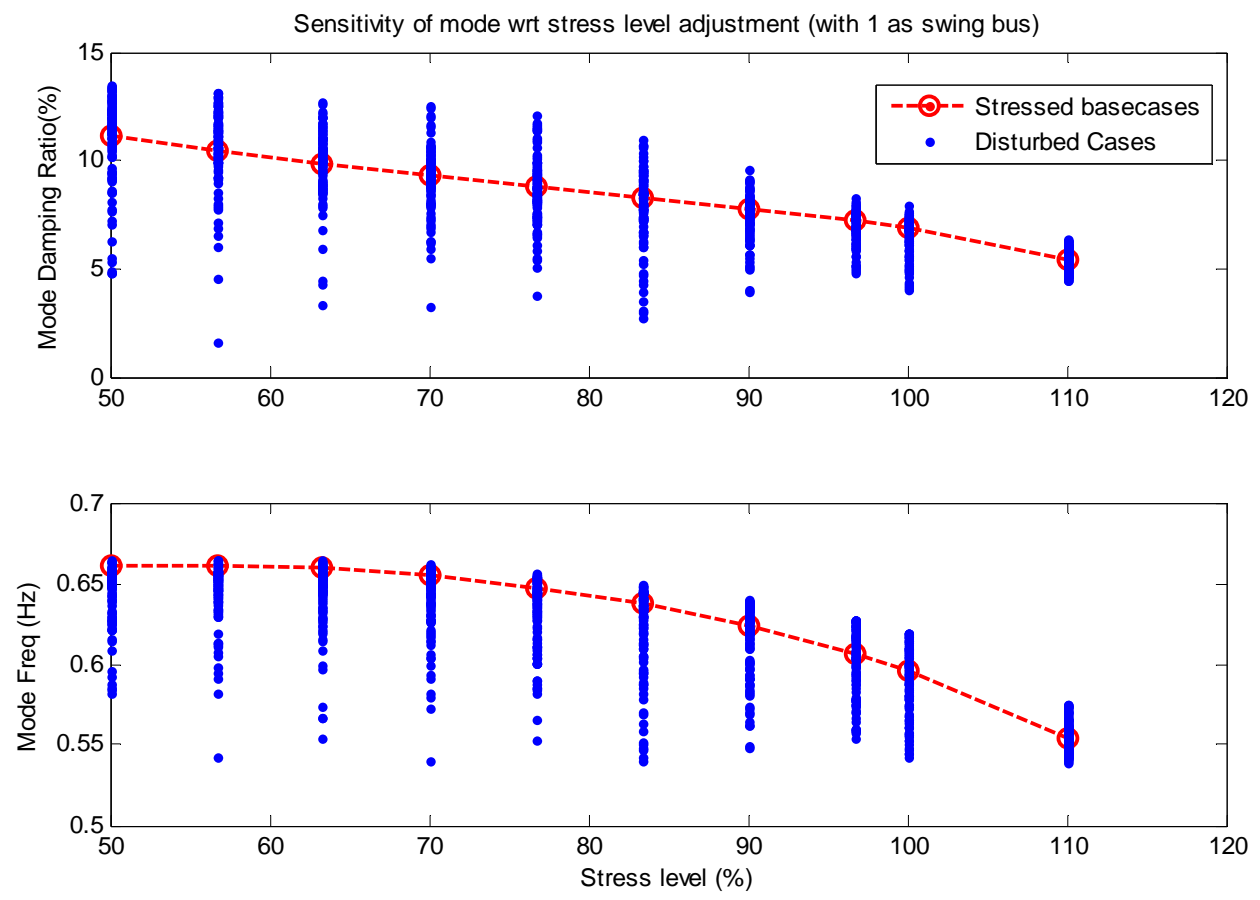

Figure 13. Correlation of the damping ratio with the system stress level (2A4M System)

The stressed cases also represent the various levels of tie line flow $P$ of Figure 12. The damping ratio decreases with the increase of the system stress level, as well as the tie line flow. This is consistent with that of the SMIB system. Heavily stressed systems are prone to small signal stability problems. From the stability improvement perspective, reducing the stress level or the tie line flow can effectively improve the damping. The tie line in this context is the oscillation path identified by the mode shape.

Figure 13 also shows that at each stress level, the mode damping and frequency can change in a wide range with the change in the generation pattern. This indicates an opportunity of improving damping through generation re-dispatch without having to shed loads.

The adjustment of tie line flow or system stress level through uniformly adjusting generation and load in a large system, at many locations, in real-time operation, would be very difficult. Shedding load is usually not a favorable option. Its practicality is limited by wide-area coordination and load serving obligations. A more practical solution would be to adjust the smallest set of selected generators with the least disturbance to scheduled transfers to achieve the desired change in damping. In the 2A4M system, all four combinations of generator pairs have been tested. The results are shown in Figure 14. For example, "G1 \& G4" denotes that adjustment of G1s power output is balanced by G4s. All the combinations result in tie line flow change, and the damping ratio is consistently correlated with the tie-lie flow level. However, Figure 14 also reveals the locational effect of the adjustment. i.e., different pairs of generators have a different effect on the damping ratio, even though they can be adjusted to achieve the same tie line flow level. Therefore, cutting tie line flow may improve damping, but depending on how the cut is performed, the effect on damping can be vastly different. For this two-area-four-machine system, the "G1 \& G4" pair is the most effective, while the "G2 \& G4" pair the least effective. 


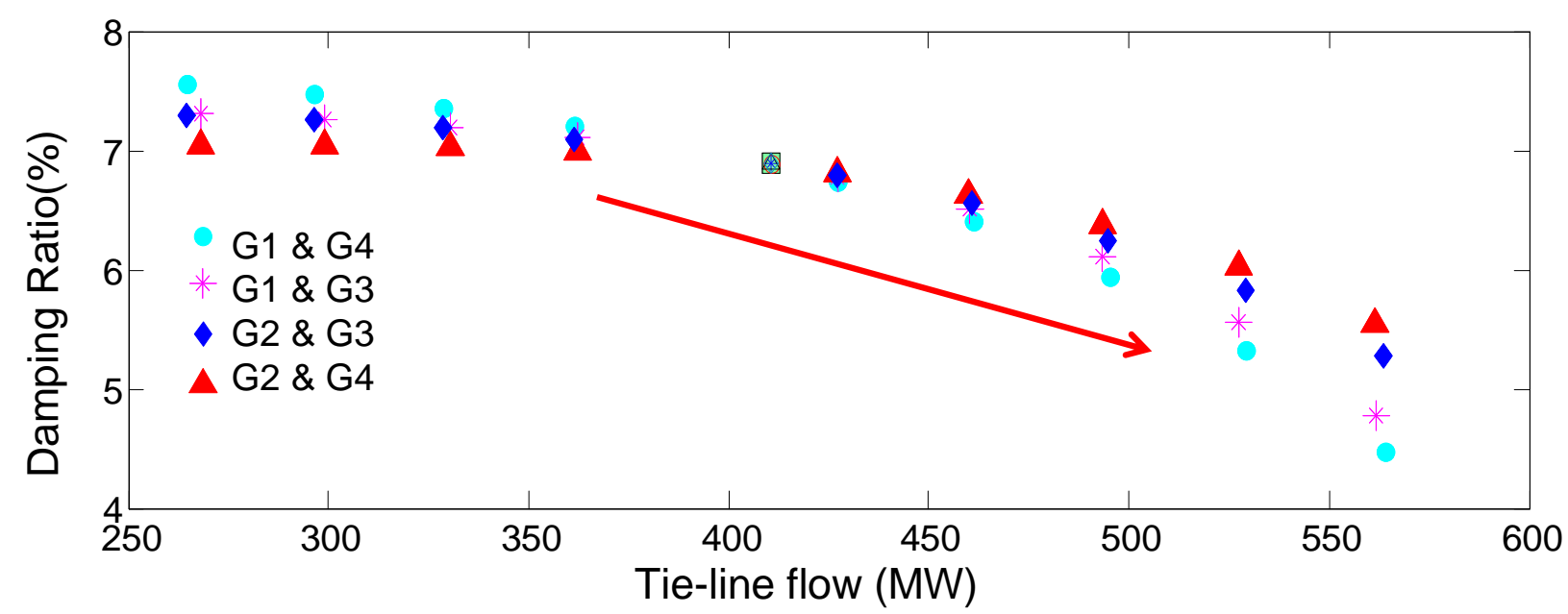

Figure 14. Correlation of the damping ratio with the tie line flow in response to generation re-dispatch of various pairs (2A4M System)

\subsubsection{7-Machine System}

With the relationship observed between generator power output adjustment on the two-area, four-machine system, the next step was to try a larger system. Rather than only having four generators, a system with 17 generators was utilized. The 17-machine model is a relatively low order rough analog to the western interconnection, or WECC reliability region. A one-line diagram of the 17-machine model is shown in Figure 15. The base modes of the 17-machine model are shown in Table 2. With multiple generators and multiple tie lines, more complex interactions are now possible. Despite having several inter-area modes to choose from, the modal interactions associated with the $0.4220-\mathrm{Hz}$ mode were the initial focus. The 17-machine model served as an intermediate step to see if the observations continued to hold for larger models.

Table 2. Selected Modal Properties of 17-machine Model

\begin{tabular}{|c|c|}
\hline Frequency $(\mathbf{H z})$ & Damping Ratio (\%) \\
\hline 0.3180 & 10.74 \\
\hline 0.4220 & 3.63 \\
\hline 0.6349 & 3.94 \\
\hline 0.6730 & 7.63 \\
\hline 0.8111 & 4.46 \\
\hline 0.9125 & 8.14 \\
\hline
\end{tabular}




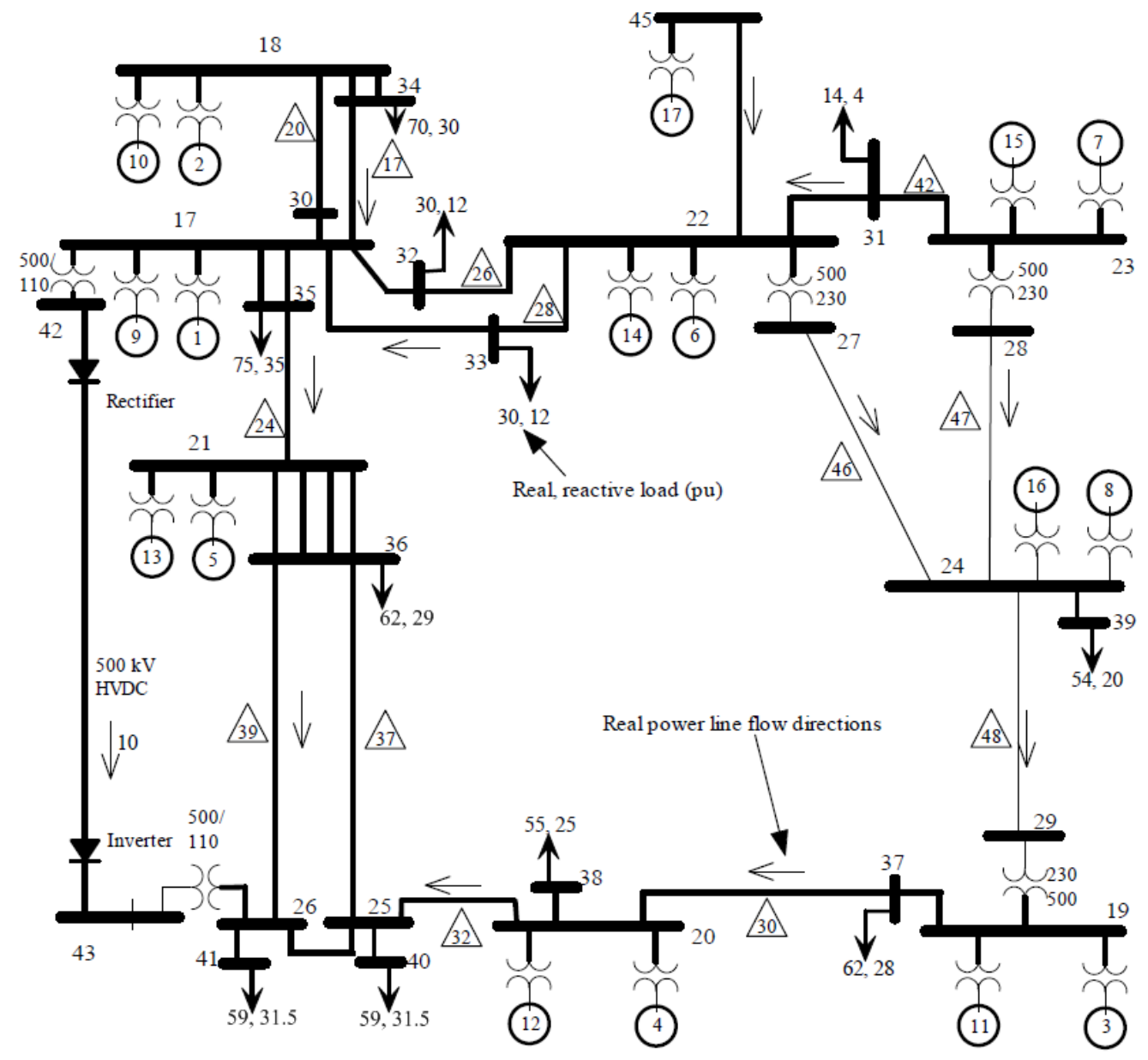

Figure 15. 17-machine system one-line diagram [28]

The same studies from the two-area, four-machine system were performed for the 17-machine system. Figure 16 plots the results of damping with respect to the tie line flow between Bus 37 and Bus 20 of the system [28]. The red squares represent stressed base cases with the same generation pattern, and the blue dots are disturbed cases with different generation patterns. It can be seen that cutting tie line flow, but maintaining the generation pattern, would be effective in damping control. However, it may not be practical for large systems, as pointed out earlier. At the same tie line flow level, the blue dots, i.e., different generation patterns, indicate the locational effect of the adjustment. It also indicates the opportunity of damping control through generation re-dispatch, as was observed for the $2 \mathrm{~A} 4 \mathrm{M}$ system. 


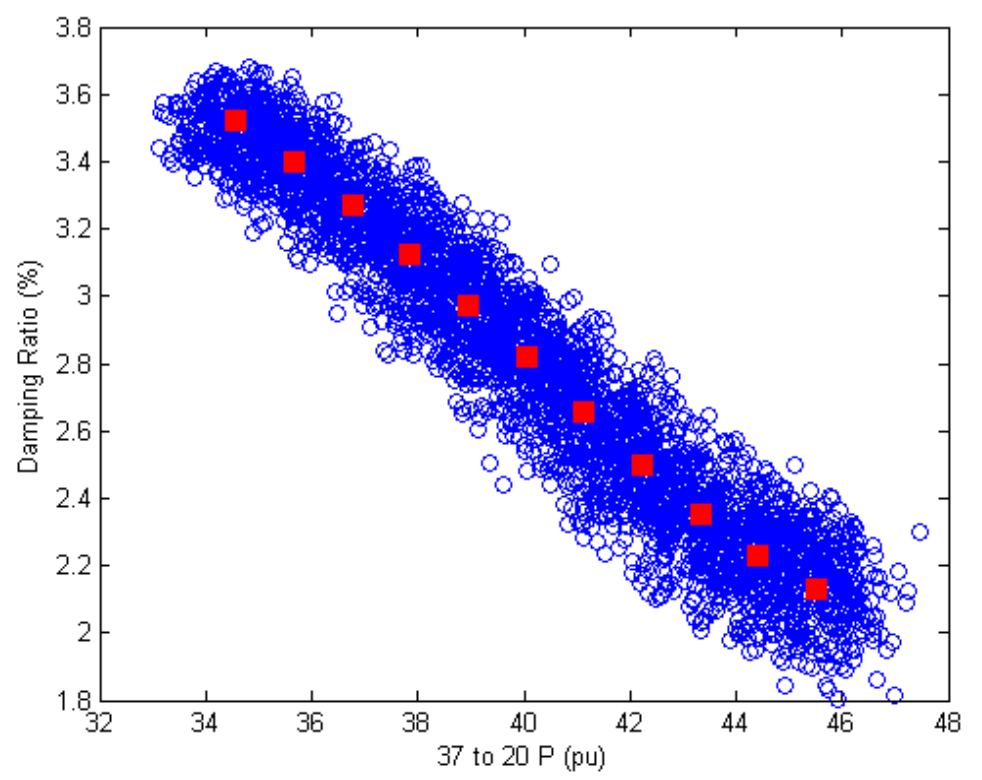

Figure 16. Correlation of damping with the tie line flow of Bus 37 - Bus 20 in the 17-machine system

\subsubsection{4-machine Model}

While the 17-machine system is a loose analog to the WECC, a more accurate model of the western U.S. power grid was necessary for small signal stability investigations. To facilitate this need, a simplified WECC system was designed, resulting in the 34-machine model. This model is more complex than the 17-machine model and attempts to model the inter-area interactions of the WECC more accurately. A selected subset of the base modes of the 34-machine model are shown in Table 3.

Table 3. Selected Modal Properties of 34-machine Model

\begin{tabular}{|c|c|}
\hline Frequency (Hz) & Damping Ratio (\%) \\
\hline 0.1652 & 12.96 \\
\hline 0.3427 & 1.41 \\
\hline 0.5105 & 8.68 \\
\hline 0.5510 & 6.24 \\
\hline 0.6052 & 0.77 \\
\hline 0.6894 & 5.54 \\
\hline 0.7221 & 5.88 \\
\hline
\end{tabular}

Of particular interest in the 34-machine model are two modes: the N-S mode and the Alberta mode. The $\mathrm{N}-\mathrm{S}$ mode is represented by the $0.1652 \mathrm{~Hz}$ and is so named because it has the northern half of the system oscillating against the southern half. The Alberta mode is represented in the $0.3427 \mathrm{~Hz}$ frequency. It is so named because it showed up in the system when Alberta was interconnected. It also has a north-south 
pattern across the western interconnect. With Alberta disconnected, the N-S and Alberta modes combine to a new single mode. This new mode has a north-south shape, is very widespread, and has a frequency somewhere between the N-S and Alberta modes described above. Like the Alberta mode with Alberta connected, the controllability of this mode is very widespread making damping control a difficult issue.

Figure 17 shows a one-line diagram of the 34-machine model. Load and generators are connected via step-up transformers. The system contains 34 generators, 122 buses, 171 lines and transformers, 54 generators, 19 load buses, and 2 DC lines. The bus, line, transformer, generator, exciter, PSS, and load models and data were selected to appropriately model the western area dynamics.

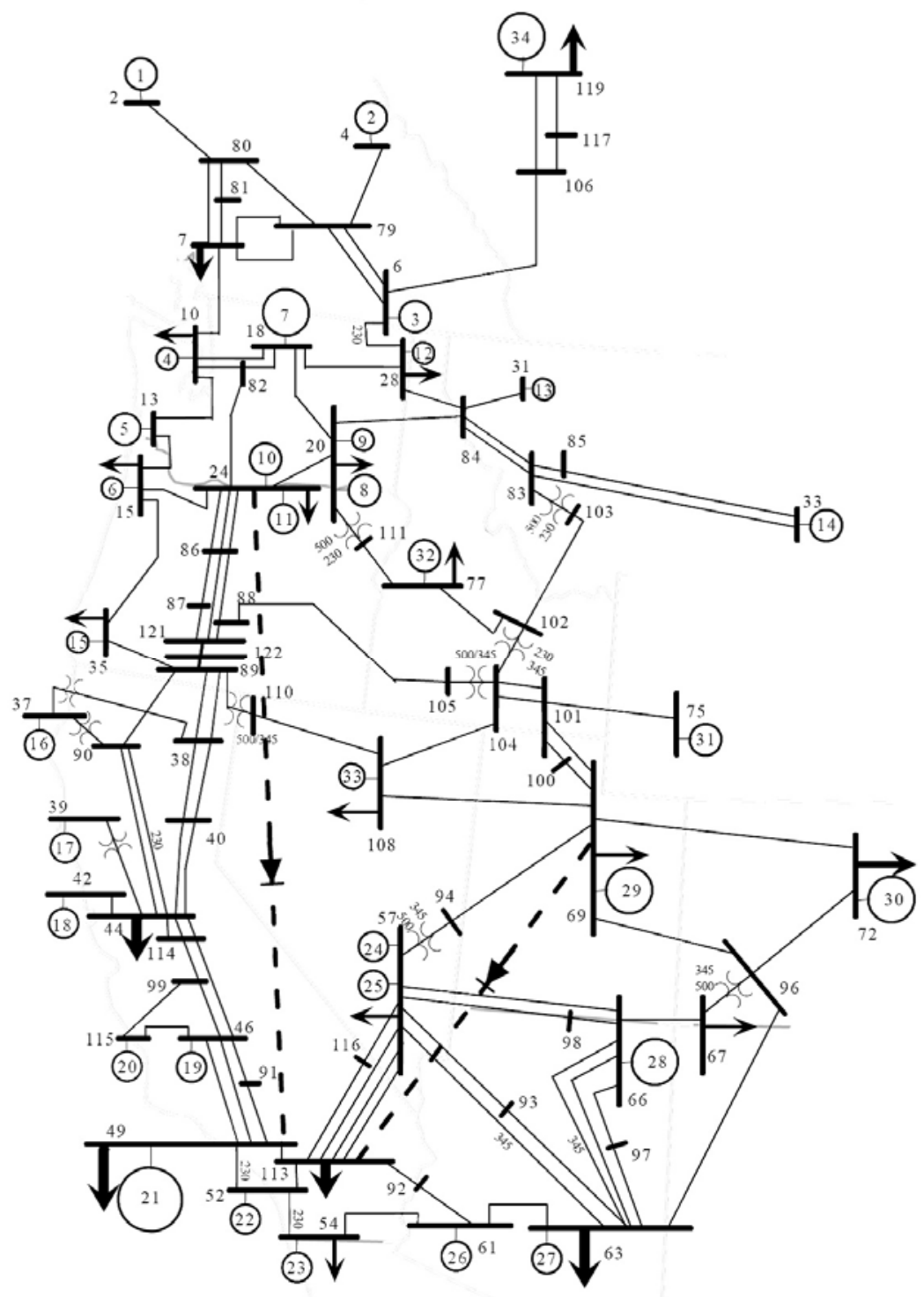

Figure 17. 34-machine model one-line diagram 
The 34-machine model was modified slightly to create various operating conditions. Table 4 and Table 5 show the results of the power flow changes and corresponding oscillatory modes:

- The " $\Delta$ mode/100MW" column shows the change in the mode for a 100-MW change in the power reference setting $\left(P_{r e f}\right)$ for the two corresponding generators. For example, the " 34 vs 7 " row shows that the percent damping of the N-S mode decreases by $2.2976 \%$ if generator 34 's power is increased by $100 \mathrm{MW}$ and generator 7's power is decreased by $100 \mathrm{MW}$.

- The " $\Delta$ mode/250MW" column shows the same as the previous major column except for a change in $P_{\text {ref }}$ of $250 \mathrm{MW}$.

As shown in Table 4, the "34 vs. 7" and "34 vs. 10" provide the most improved damping for both the 100 MW and $250 \mathrm{MW}$ changes in $P_{\text {ref, }}$ with respect to the North-South mode. Table 5 shows how the changes affecting the Alberta mode also favor the " 34 vs. 10" generator adjustment, but other options provide nearly the same benefit.

A further detailed study for this system [60] concluded:

- The optimal generator set(s) for reducing a given mode's damping relates to reducing the flows on a small set of transmission interties. The intertie ends are not necessarily between mode-shape areas. The generators' adjusted on the two sides of an intertie are not unique. One can select nearly any generator in a given area.

- The relationship between a given mode's damping and intertie flows is very consistent when comparing several power-flow conditions. That is, a given mode's damping is always reduced by reducing the flow on the same set of interties independent of the power-flow condition.

These conclusions lead one to the following relatively simple operational control strategy for increasing modal damping:

1. On-line and off-line studies are conducted to determine the key intertie relations for a given mode. The results from this study conclude that these key interties will not change unless the grid topology changes.

2. The given mode's frequency, damping, and shape are monitored on a continuous basis using mode-meter technology, and the system's topology is monitored. The frequency and shape information is required to make sure one is monitoring the desired mode. If the damping falls below acceptable levels, generator real-power set points are adjusted on sets of generators on each side of the key interties in order to reduce the intertie flows. 
Table 4. 34-machine Model, N-S Mode

\begin{tabular}{|c|r|r|r|r|r|r|}
\hline$\Delta \mathrm{P}$ & \multicolumn{3}{|c|}{$\Delta$ mode / $100 \mathrm{MW}$} & \multicolumn{3}{c|}{$\Delta$ mode / $250 \mathrm{MW}$} \\
\hline gen vs gen & \multicolumn{1}{|c|}{$\Delta$ real } & \multicolumn{1}{|c|}{$\Delta \mathrm{f}(\mathrm{Hz})$} & \multicolumn{1}{c|}{$\Delta \% \mathrm{D}$} & \multicolumn{1}{|c|}{$\Delta$ real } & \multicolumn{1}{c|}{$\Delta \mathrm{f}(\mathrm{Hz})$} & \multicolumn{1}{c|}{$\Delta \% \mathrm{D}$} \\
\hline 34 vs 7 & -0.023234 & 0.008792 & 2.2976 & -0.060666 & 0.032259 & 7.151 \\
\hline 34 vs 10 & -0.023246 & 0.008824 & 2.2999 & -0.060588 & 0.032312 & 7.1472 \\
\hline 14 vs 7 & -0.00028457 & 0.000271 & 0.031032 & -0.00099338 & 0.000771 & 0.10417 \\
\hline 14 vs 10 & -0.00033773 & 0.000311 & 0.036552 & -0.0011602 & 0.000878 & 0.12115 \\
\hline 10 vs 18 & $-1.81 \mathrm{E}-05$ & 0.000192 & 0.006376 & $-8.08 \mathrm{E}-05$ & 0.000499 & 0.019471 \\
\hline 10 vs 21 & 0.00025205 & 0.000224 & -0.01577 & 0.00058337 & 0.000587 & -0.03486 \\
\hline 7 vs 18 & $-6.92 \mathrm{E}-05$ & 0.000231 & 0.011705 & -0.0002215 & 0.000603 & 0.034091 \\
\hline 7 vs 21 & 0.00020081 & 0.000264 & -0.01042 & 0.00044098 & 0.000692 & -0.02008 \\
\hline
\end{tabular}

Note: Red numbers indicate negative quantities

Table 5. 34-machine Model, Alberta Mode

\begin{tabular}{|c|c|c|c|c|c|c|}
\hline$\Delta \mathrm{P}$ & \multicolumn{3}{|c|}{$\Delta$ mode / $100 \mathrm{MW}$} & \multicolumn{3}{|c|}{$\Delta$ mode / $250 \mathrm{MW}$} \\
\hline gen vs gen & $\Delta$ real & $\Delta f(H z)$ & $\Delta \% \mathrm{D}$ & $\Delta$ real & $\Delta \mathrm{f}(\mathrm{Hz})$ & $\Delta \% \mathrm{D}$ \\
\hline 34 vs 7 & -0.0086 & 0.005607 & 0.46408 & -0.01895 & 0.016138 & 1.0826 \\
\hline 34 vs 10 & -0.00934 & 0.005768 & 0.50199 & -0.02106 & 0.016593 & 1.1956 \\
\hline 14 vs 7 & -0.00963 & 0.001739 & 0.4846 & -0.03952 & 0.005033 & 1.9935 \\
\hline 14 vs 10 & -0.01038 & 0.001899 & 0.52248 & -0.04199 & 0.005479 & 2.1217 \\
\hline 10 vs 18 & -0.00529 & 0.001064 & 0.26646 & -0.0136 & 0.002732 & 0.68799 \\
\hline 10 vs 21 & -0.00561 & 0.001253 & 0.28354 & -0.01451 & 0.00323 & 0.73727 \\
\hline 7 vs 18 & -0.00596 & 0.001218 & 0.30026 & -0.01527 & 0.003128 & 0.77386 \\
\hline 7 vs 21 & -0.00628 & 0.001407 & 0.31738 & -0.01619 & 0.003628 & 0.82343 \\
\hline
\end{tabular}

Note: Red numbers indicate negative quantities 


\subsection{Vision on the MANGO Procedure}

Extensive studies shown in the previous section provide strong evidence that small-signal stability can be effectively improved by adjusting the power system operating point. To enable the adjustment, an operational MANGO procedure needs to be established to guide operators in real-time power system operation. The procedure needs to include three major steps:

1) Recognition - operator recognizes the need for operating point adjustment;

2) Implementation - operator implements the adjustment per recommendations by the MANGO approach; and

3) Evaluation - operator evaluates the effectiveness of the adjustment and repeats the procedure of necessary.

\subsection{The MANGO Procedure}

Consistent with these three steps, the following MANGO procedure is proposed for improving smallsignal stability:

1) Estimate oscillation modes and mode shapes using ModeMeter based on real-time phasor measurements;

2) Observe the mode damping and mode energy, as well as its oscillation paths through its mode shape;

3) Recognize the need for operating point adjustment when the damping is below a pre-specified threshold and the oscillation energy exceeds a threshold;

4) Implement tie line flow reduction of the oscillation path with a pre-defined amount or percentage. The tie lines are identified by the mode shapes. This is the preliminary level of implementation. Uncertainties caused by locational effect make it difficult to predict the effectiveness and may even cause undesired results;

5) Identify the sensitivity relationship between the modes of interest and operating parameters. Such a relationship is referred to as a MANGO model;

6) Generate recommendations for operating point adjustment based on the MANGO model and a damping target. The MANGO recommendations are presented to operators as options to change operating parameters. A sample recommendation would be: "Generator A's output needs to be reduced by $200 \mathrm{MW}$ and Generator B's output increased by $200 \mathrm{MW}$ to improve damping from $1 \%$ to $5 \%$ ".

7) Conduct feasibility test to ensure that the recommended adjustment would result in a solvable power flow and not violate any generation capacity and other limits; 
8) Implement the adjustment with consideration of other metrics, such as transient stability and voltage stability. This is the advanced-level implementation because it provides more specific actions and more predictable results;

9) Monitor the change in modes and mode shapes from ModeMeter;

10) Evaluate the effectiveness by observing whether the damping is adequate and how accurate the MANGO procedure predicts the results. Repeat the MANGO procedure if damping does not achieve the desired level.

Figure 18 presents the overall MANGO framework.

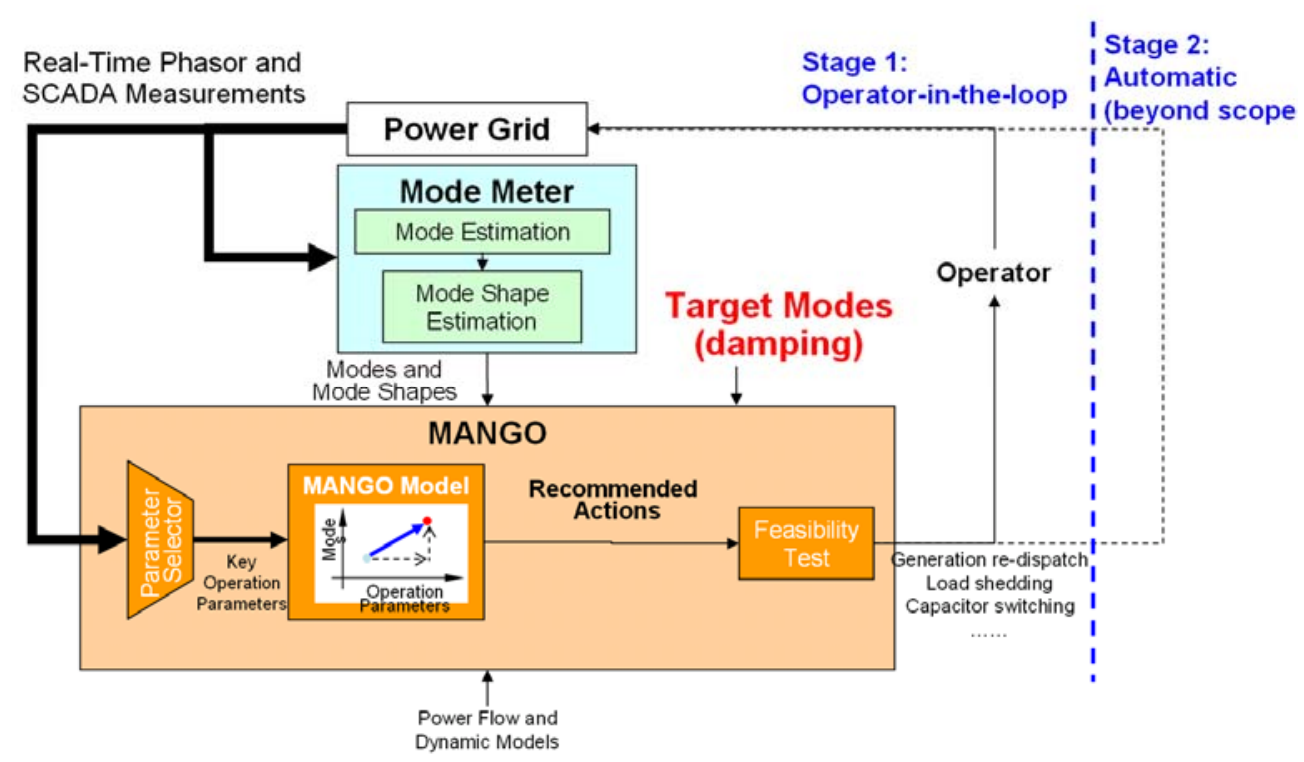

Figure 18. Proposed MANGO framework

\subsection{Implementation Consideration}

The proposed MANGO procedure can be integrated in current operating procedures, so there would not be new procedures created solely for MANGO. This way, the complexity added to the operational environment is minimized, and it would be easier for grid operators to accept an add-on function rather than a completely new procedure.

Several existing operating procedures can be considered for MANGO integration. The Bonneville Power Administration (BPA) currently uses a dispatcher standing order (DSO) 303 for mitigating system oscillation problems. In the general case, the DSO calls for reductions in tie line transfer on the affected path by increments of $10 \%$ until the damping is satisfactory when oscillations are observed. The DSO gives very limited information on adjustment generators and does not estimate the expected change in damping for a $10 \%$ adjustment. The MANGO procedure can be used to enhance the DSO 303 with specifics such as locations, sizes of adjustment, and expected damping improvement. It can also enhance the DSO 303 with the inclusion of other oscillation modes. 
Current North American Electric Reliability Corporation (NERC) operating procedures such as TOP-004 [29][30], TOP-007 [31][32], TOP-008 [33][34], and IRO-006[35][36] can also be used to integrate MANGO recommendations. TOP-004 is to ensure interconnection reliability operating limits (IROLs) and system operating limits (SOLs) are satisfied, while TOP-007 defines the reporting procedure if IROLs or SOLs are violated. TOP-008 requires the transmission operator experiencing or contributing to an IROL or SOL violation shall take immediate steps to relieve the condition, which may include shedding firm load. Small-signal stability limits are part of the IROLs, and MANGO recommendations align with the requirements in TOP-008. IRO-006 is a tie line relief (TLR) standard. It defines the procedures for adjusting interchange transactions, network and native load contributions and market dispatch contributions to relieve overloads on the transmission facilities modeled in the interchange distribution calculator (IDC). MANGO recommendations can be used to relieve transmission overloads per the metric of oscillations.

For the WECC, the selection of generators may consider existing E-Tags when generating and implementing the MANGO recommended actions. E-Tags are used to define transactions with generators grouped for providing energy services [37]. Generation re-dispatch according to the E-Tag generator groups enables the use of established channels to influence energy transactions.

At the first stage of implementation, the MANGO procedure is a measurement-based and operator-in-theloop procedure, as shown in Figure 18. The MANGO model can be updated according to the current measurement and mode estimation results. Operators are included into the loop to bring in expert knowledge. In the future, after the effectiveness of the MANGO procedure is validated, a greater degree of automation may be possible. For example, the automatic process might be integrated as part of a remedial action scheme (RAS) system or a special protection system (SPS).

\subsection{Modal Sensitivity}

The recognition and evaluation steps in the MANGO procedure rely on a good ModeMeter to estimate the current modes, while the implementation step builds on modal sensitivity, i.e., the relationship of oscillation modes and operating parameters. The relationship is generally nonlinear, and thus it is impractical to derive a closed-form analytical solution for this relationship. Theoretically, the sensitivity can be calculated from the model of a power system, but the model is usually not able to reflect real-time operating conditions. Therefore, this work has been primarily centered on estimating modal sensitivity from real-time measurement. This is the focus of the remainder of this report. Eigenvalue-theory-based sensitivity has been studied and concluded to be not applicable for real-time estimation. Many items in the eigen-sensitivity equations cannot be estimated with regular phasor measurements. Three approaches for estimating modal sensitivities are then proposed and studied to approximate the sensitivity relationship. These approaches are relative sensitivity estimation, ANN-based nonlinear mapping, and decision-tree-based rule derivation. The relative sensitivity is formulated using least square principles in the form that can be estimated directly from measurement. The testing has been carried out with a medium-sized system. The results indicate how strong generation output relates to the damping, giving clear guidance on which generator needs to be adjusted to improve damping. ANN-based nonlinear mapping has been studied, and it can successfully approximate the modal sensitivity from testing results with medium-sized systems. In addition, a decision-tree-based method is studied to summarize the relationship between operator controllable variables and modes. 
The next three sections present further details on the three MANGO methods. System topology changes the inherent dynamics in a power system, and thus changes the modal properties. Topology analysis is conducted to characterize the impact of topology change in oscillation modes, which is presented in Section 8 . 


\subsection{MANGO Method 1: Relative Modal Sensitivity}

The studies in the previous section clearly indicate the opportunity of operating point adjustment for damping improvement, as well as the locational effect of the adjustment. A key step in the method is to quantify the sensitivity of damping to operating parameters such as generation and load. Modal sensitivity studies have been used to study most influential factors in inter-area oscillations [38], as well as to identify the proper locations for PSS [13] and FACT devices [12]. This section explores the sensitivity from the eigenvalue theory perspective and introduces the concept of relative modal sensitivity. The objective is to identify the right operating parameters for adjustment. In some cases, especially for small systems, engineering experience can provide satisfactory selection of parameters for operation actions. However, experience-based selection may not identify the most effective options for large-scale complex systems, such as the western U.S. power grid. Systematic methods have yet to be developed for selecting locations for damping improvement.

\subsection{Modal Sensitivity Derived from Eigenvalue Theory}

Eigenvalue analysis techniques are used on an explicit or approximate model of the system. The modelbased analysis is conducted in a simulation environment. Therefore, all of the state variables are known and available. This allows the eigenvalues, and by extension the frequency and damping ratio, to be calculated directly. It further allows the calculation of eigenvalue sensitivity to operating parameters of interest. Following Equation (2), the basic eigenvalue sensitivity is given by [7]:

$$
\frac{\partial \lambda}{\partial p}=\boldsymbol{\Psi} \frac{\partial \mathbf{A}}{\partial p} \boldsymbol{\Phi}
$$

where $\lambda$ represents the eigenvalues of the system, $\Phi$ and $\Psi$ denote the right and left eigenvectors, respectively, and $p$ represents the operating parameter of interest, e.g., generation or load. To reduce the computation time for a large power grid, Smed [39] proposed a sparse method to calculate the modal sensitivity. In Rommes and Martin [40], a sensitive pole method is proposed to identify the poles that are most sensitive to parameter changes. To arrive at a practical solution, the sensitivity must be calculated as a numerical approximation through perturbation. Under this assumption, the eigenvalue sensitivity becomes:

$$
\frac{\partial \lambda}{\partial p}=\boldsymbol{\Psi} \frac{\Delta \mathbf{A}}{\Delta p} \boldsymbol{\Phi}
$$

This basic modal sensitivity method has been applied to the 17-machine system [28]. The results of the 0.4-Hz modal sensitivity, with respect to generator power output, are shown in Figure 19. The two largest sensitivities are associated with generator 17 and generator 10 . Increasing generator 10 power will decrease the damping ratio, while increasing generator 17 power will increase the damping ratio. Generators 17 and 10 would be the most effective pair to use for generation re-dispatch to improve the 0.4-Hz mode damping. The sensitivity information also indicates how much change in damping can be expected by the generator power adjustment. 


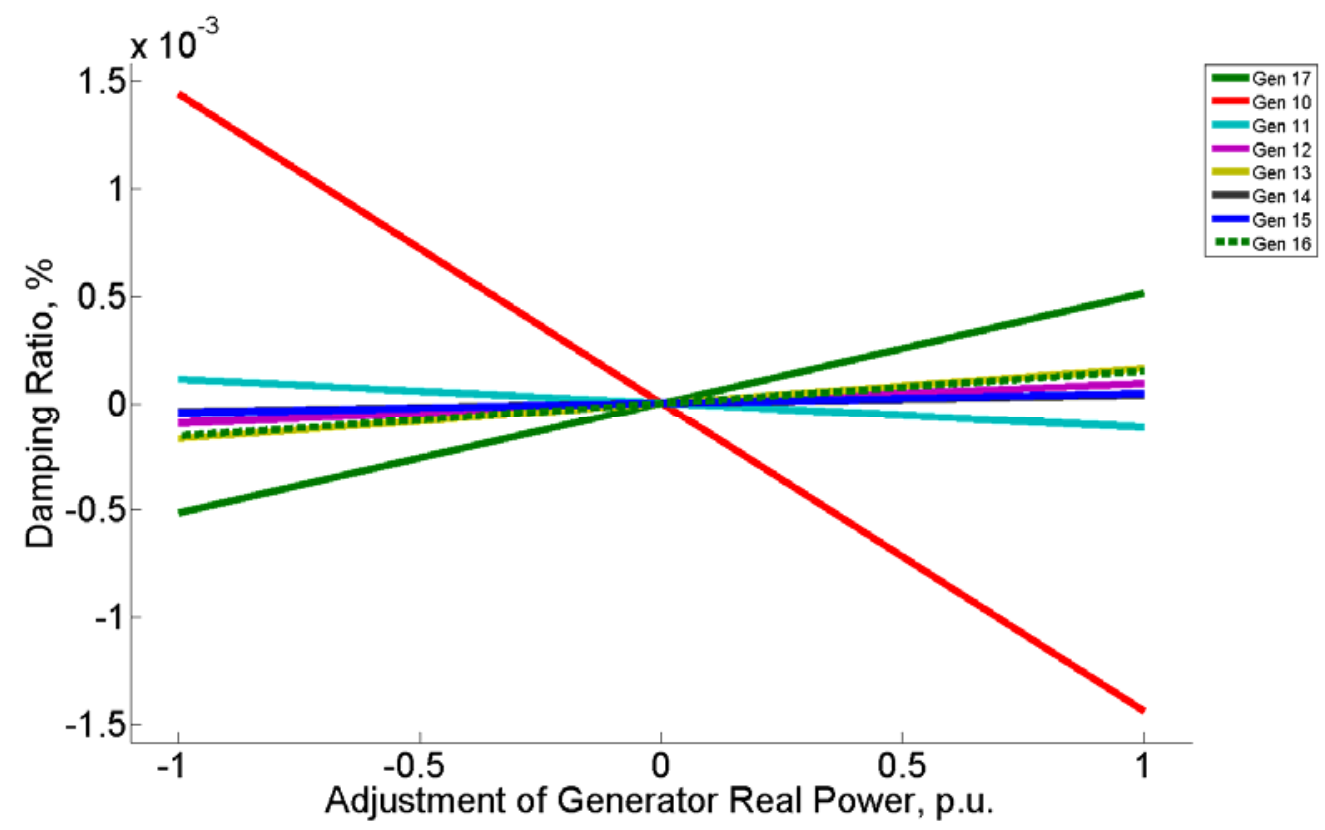

Figure 19. Modal sensitivity of the $0.4-\mathrm{Hz}$ mode in the 17 -machine system

Although the sensitivity results match observations from other studies, as presented in Huang et al. [41], it has one major shortcoming. During the perturbation process, the change of generation or load is absorbed by the swing bus. The sensitivity is affected by the choice of the swing bus. In the 17-machine system, generator 10 is relatively close to the swing bus. If the swing bus was moved to a further geographical bus location, the interactions would be different, and the sensitivity is expected to change as well. To overcome this issue, the concept of relative modal sensitivity is proposed.

\subsection{Relative Modal Sensitivity}

The eigenvalue-theory-based sensitivity works well to characterize the impact of independent variables. However, in the context of operating point adjustment, it involves at least two variables. For example, a generation increase at one location will need to be balanced by a generation decrease or a load increase at another location. The effect of the adjustment on modal damping depends on how the pair is chosen. In another words, modal sensitivity of one location is always relative to how the other location is chosen.

The relative modal sensitivity method still uses perturbation, but rather than perturbing a parameter and allowing the swing bus to absorb the difference, this sensitivity method attempts to disperse the difference across other locations. This effectively minimizes the nonlinear effect of the fixed swing bus on the sensitivity. That is, if the real power output of a generator is increased by $5 \mathrm{MW}$, then all the other generators in the system will be decreased slightly to balance the extra $5 \mathrm{MW}$ of generation.

For a general system with $N$ generator and load locations, perturb real power by $\Delta P$ at one location. Allocating the perturbation evenly at all other locations results in a change in the eigenvalue that can be expressed as: 


$$
\begin{aligned}
\Delta \lambda^{(1)} & =\frac{\partial \lambda}{\partial P_{1}} \Delta P_{1}+\frac{\partial \lambda}{\partial P_{2}} \Delta P_{2}+\ldots+\frac{\partial \lambda}{\partial P_{N}} \Delta P_{N} \\
& =\frac{\partial \lambda}{\partial P_{1}} \Delta P-\frac{\partial \lambda}{\partial P_{2}}\left(\frac{\Delta P}{N-1}\right)-\ldots-\frac{\partial \lambda}{\partial P_{N}}\left(\frac{\Delta P}{N-1}\right)
\end{aligned}
$$

The same magnitude perturbation applied to a different location would result in:

$$
\begin{aligned}
\Delta \lambda^{(2)} & =\frac{\partial \lambda}{\partial P_{1}} \Delta P_{1}+\frac{\partial \lambda}{\partial P_{2}} \Delta P_{2}+\ldots+\frac{\partial \lambda}{\partial P_{N}} \Delta P_{N} \\
& =-\frac{\partial \lambda}{\partial P_{1}}\left(\frac{\Delta P}{N-1}\right)+\frac{\partial \lambda}{\partial P_{2}} \Delta P-\ldots-\frac{\partial \lambda}{\partial P_{N}}\left(\frac{\Delta P}{N-1}\right)
\end{aligned}
$$

The difference of Equations (10) and (11) is

$$
\Delta \lambda^{(1)}-\Delta \lambda^{(2)}=\left(\frac{\partial \lambda}{\partial P_{1}}-\frac{\partial \lambda}{\partial P_{2}}\right)\left(\frac{N}{N-1}\right) \Delta P
$$

Relative sensitivity between locations 1 and 2 can then be derived as

$$
\left(\frac{\partial \lambda}{\partial P_{1}}-\frac{\partial \lambda}{\partial P_{2}}\right)=\left(\frac{N-1}{N}\right) \frac{\Delta \lambda^{(1)}-\Delta \lambda^{(2)}}{\Delta P}
$$

This equation clearly shows the relative nature of the modal sensitivity for operating condition adjustment. If all locations are perturbed, applying Equation (13) to all the perturbations can generate $N-1$ relative sensitivities:

$$
\left(\frac{\partial \lambda}{\partial P_{1}}-\frac{\partial \lambda}{\partial P_{2}}\right),\left(\frac{\partial \lambda}{\partial P_{3}}-\frac{\partial \lambda}{\partial P_{2}}\right), \ldots,\left(\frac{\partial \lambda}{\partial P_{N}}-\frac{\partial \lambda}{\partial P_{2}}\right)
$$

Other relative sensitivity can be obtained directly by differentiating two related relative sensitivities. For example,

$$
\left(\frac{\partial \lambda}{\partial P_{1}}-\frac{\partial \lambda}{\partial P_{3}}\right)=\left(\frac{\partial \lambda}{\partial P_{1}}-\frac{\partial \lambda}{\partial P_{2}}\right)-\left(\frac{\partial \lambda}{\partial P_{3}}-\frac{\partial \lambda}{\partial P_{2}}\right)
$$

Note that an alternative way of computing relative sensitivity is to form a basic set of generator pairs, as in Equation (14), and compute the relative sensitivity directly. This alternative approach is simple and will result in the same relative sensitivity estimation as the linearized sensitivity equations, such as Equations (10) and (11). In reality, because of nonlinearity, an error term will appear on the right side of the sensitivity equations. Thus, it is proposed that the system be perturbed in such a way that the eigenvalue changes are mainly caused by only one generation change. The proposed approach helps reduce the influence of nonlinear effects from all other generators in that the perturbations are dispersed, and thus are relatively small in all other generators.

Figure 20 shows the results of the relative sensitivity method as applied to both generator and load locations in the 17-machine system. All the sensitivities are relative to the swing bus used in the previous section. Figure 20 shows that the sensitivities are identical to those obtained in the previous section in Figure 19. Because the swing bus absorbs the power imbalance, the sensitivities are relative to the swing 
bus generator. Therefore, the sensitivities obtained in the previous section are actually relative sensitivities between other generators and the swing bus generator.

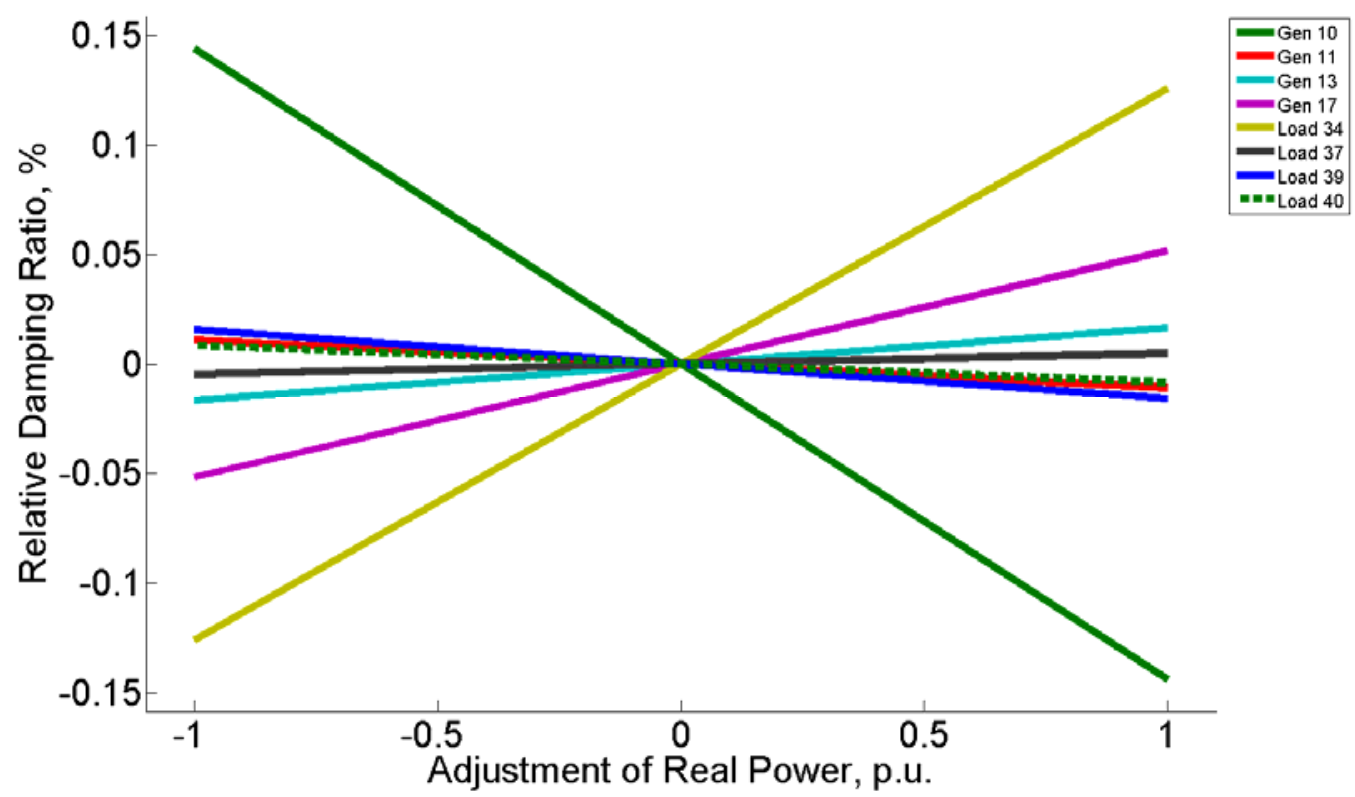

Figure 20. Relative modal sensitivity of the $0.4 \mathrm{~Hz}$ mode in the 17 -machine system

The most significant importance of the relative sensitivity concept is that it provides the insight for guiding operating condition adjustment to improve damping. In other words, to improve damping, the most effective pair are the locations with the largest sensitivity differences. For the 17-machine system, the pair is generator 10 (the largest negative slope) and the load at bus 34 (the largest positive slope). Decreasing 1-p.u. generation at generator 10 and increasing 1-p.u. load at bus 34 would increase the damping ratio by $0.275 \%[=0.15 \%-(-0.125 \%)]$. This requires increasing the load, which may not be practical. If load adjustment is not preferred, as in many situations, the most effective generator pair is the generators 10 and 17. A 1-p.u. adjustment (generator 10 decrease and generator 17 increase) would result in damping increase of $0.2 \%[=0.15 \%-(-0.05 \%)]$.

\subsubsection{Relative Sensitivity Estimation from Real-Time Measurement}

In on-line damping control applications, models are usually not available or not accurate for the real-time condition. Perturbing a model to obtain relative sensitivities is not practical. It is desirable to estimate relative modal sensitivities from real-time measurements. Equation (10) can be expanded to formulate the relative sensitivity estimation problem.

In a general power system, first assume power measurements $P_{i}$ at the locations of generators and loads are available, and modes can be estimated from measurements using mode meter techniques [9]. Mode change from one time instant to another can be characterized as a linear combination of the effect of power change at all the locations:

$$
\Delta \lambda^{(i)}=\frac{\partial \lambda}{\partial P_{1}} \Delta P_{1}^{(i)}+\frac{\partial \lambda}{\partial P_{2}} \Delta P_{2}^{(i)}+\ldots+\frac{\partial \lambda}{\partial P_{N}} \Delta P_{N}^{(i)}+\varepsilon^{(i)}
$$


This is a generalized version of Equation (10): power change is not evenly distributed, and a noise term is included to account for noise in the measurement. Given a time series of measurements, $M$ equations can be formulated:

$$
\left[\begin{array}{c}
\Delta \lambda^{(1)} \\
\Delta \lambda^{(2)} \\
\vdots \\
\Delta \lambda^{(M)}
\end{array}\right]=\left[\begin{array}{cccc}
\Delta P_{1}^{(1)} & \Delta P_{2}^{(1)} & \cdots & \Delta P_{N}^{(1)} \\
\Delta P_{1}^{(2)} & \Delta P_{2}^{(2)} & \cdots & \Delta P_{N}^{(2)} \\
\vdots & \vdots & \ddots & \vdots \\
\Delta P_{1}^{(M)} & \Delta P_{2}^{(M)} & \cdots & \Delta P_{N}^{(M)}
\end{array}\right]\left[\begin{array}{c}
\frac{\partial \lambda}{\partial P_{1}} \\
\frac{\partial \lambda}{\partial P_{2}} \\
\vdots \\
\frac{\partial \lambda}{\partial P_{N}}
\end{array}\right]+\left[\begin{array}{c}
\varepsilon^{(1)} \\
\varepsilon^{(2)} \\
\vdots \\
\varepsilon^{(M)}
\end{array}\right]
$$

Note the power balance condition:

$$
\sum P_{i}=\Delta P_{1}^{(i)}+\Delta P_{2}^{(i)}+\cdots+\Delta P_{N}^{(i)}=0
$$

Eliminating one power measurement, say $P_{1}$, from Equation (17) results in an independent set of equations:

$$
\left[\begin{array}{c}
\Delta \lambda^{(1)} \\
\Delta \lambda^{(2)} \\
\vdots \\
\Delta \lambda^{(M)}
\end{array}\right]=\left[\begin{array}{cccc}
\Delta P_{2}^{(1)} & \Delta P_{3}^{(1)} & \cdots & \Delta P_{N}^{(1)} \\
\Delta P_{2}^{(2)} & \Delta P_{3}^{(2)} & \cdots & \Delta P_{N}^{(2)} \\
\vdots & \vdots & \ddots & \vdots \\
\Delta P_{2}^{(N)} & \Delta P_{3}^{(N)} & \cdots & \Delta P_{N}^{(M)}
\end{array}\right]\left[\begin{array}{c}
\frac{\partial \lambda}{\partial P_{2}}-\frac{\partial \lambda}{\partial P_{1}} \\
\frac{\partial \lambda}{\partial P_{3}}-\frac{\partial \lambda}{\partial P_{1}} \\
\vdots \\
\frac{\partial \lambda}{\partial P_{N}}-\frac{\partial \lambda}{\partial P_{1}}
\end{array}\right]+\left[\begin{array}{c}
\varepsilon^{(1)} \\
\varepsilon^{(2)} \\
\vdots \\
\varepsilon^{(M)}
\end{array}\right]
$$

If $M>N$, a least square method can be used to estimated the $N-1$ relative sensitivities. This process only needs to run once for one operating condition, and all relative sensitivities can be calculated according to Equation (15). There is no need to enumerate all the $N(N-1) / 2$ pairs of generators and loads. The largest relative sensitivities of the $N(N-1) / 2$ pairs can then derive the appropriate MANGO recommendations.

The derivation and estimation process does not rely on a specific topology, so conceptually it is applicable to any power systems. With the increase of system size, the dimension of the matrix in Equation (19) increases and the computation time increases, but the principle holds. Some implementation issues include the robustness of the method in rejecting measurement noise, coordination for recommended adjustment, topology change impact on the effectiveness, and multi-mode interaction. It is also worth exploring the combination of three or more locations, especially when one pair of locations is limited by adjustment capacity. All these are topics that are worthy of further research and many of them are part of ongoing work. Relevant results will be reported in future publications.

\subsubsection{Case Studies Using the Two-Area, Four-Machine System}

To verify the performance of the proposed algorithm in estimating modal sensitivities, the two-area-fourmachine system shown in Figure 12 is used to generate simulation data sets. The following study was carried out with the Power System Toolbox (PST) version 3.0 [14][42]. The base case of the two-areafour-machine system is originally from the Power System Toolbox, and stored in "data2a.m". For the base case, the system has an inter-area mode at Freq $=0.60 \mathrm{~Hz} \mathrm{~Hz}$ and $\mathrm{DR}=6.89 \%$, which is chosen as the mode of interest. To simulate the random load and generation variation, random perturbations are added to the generators and loads to generate 300 cases. G1 is chosen as the reference for calculating relative 
modal sensitivity. Two groups of data are generated with different levels of perturbation. The first group has $1 \%$ of load and generation perturbations, while the second group has $10 \%$ of load and generation perturbations. The PST is used for calculating eigenvalues. For simulation, the mode sensitivity can be calculated directly from the model and is used as the reference for evaluating the performance of the model sensitivity estimation algorithm. To unify the sign notation of load and generation, the loads are denoted as negative generation. The study results for damping ratio sensitivity analysis are summarized in Figure 21. The sensitivity is relative to the reference generator G1. It can be observed that the estimation results are consistent with direct computation. This consistency verifies the validity of the proposed algorithm. It is also observed that the estimation error increases with the level of perturbation. The damping ratio sensitivity estimation from $1 \%$ perturbation carries slightly less error than the sensitivity estimation from $10 \%$ changes, which indicates the nonlinear behavior introduced by the larger perturbation and not modeled in the linearized method.

\section{Damping Ratio (Gen 1 as reference)}

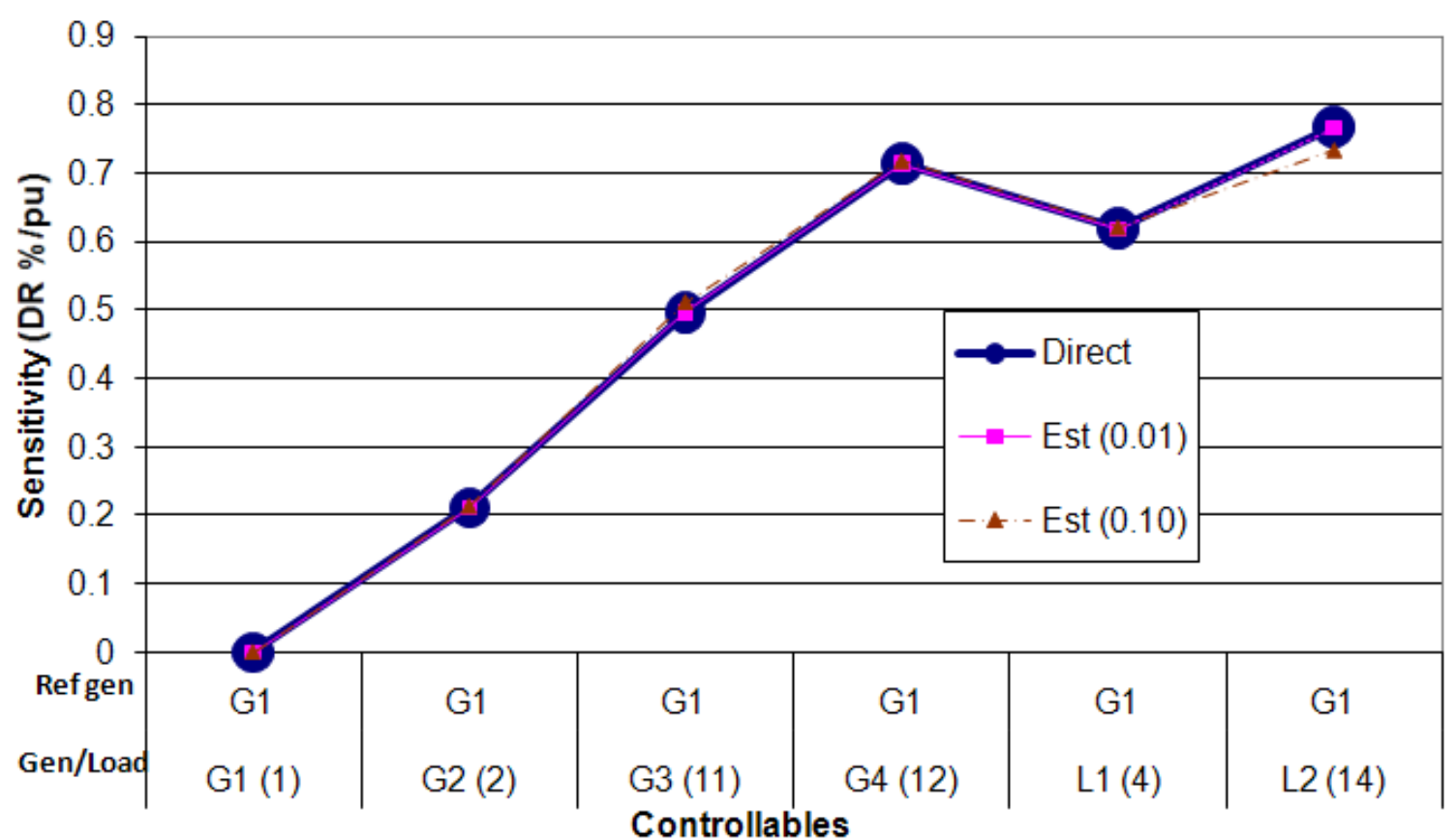

Figure 21. Estimated modal sensitivity of the two-area, four-machine system

To apply the analysis results shown in Figure 21 for generating a MANGO recommendation, the largest sensitivity difference should be examined when identifying the most effective generator pair. Figure 21 indicates that the most effective pair is G4 and G1, with the largest sensitivity difference being $0.7 \%$ per p.u. power. The corresponding MANGO recommendation is "increase the G4 power output by 3 p.u. and decrease G1 by 3 p.u., and the damping ratio is expected to increase by about $2.1 \%$ ( 3 p.u. * $0.7 \%$ per p.u.)".

To further verify this MANGO recommendation, a time domain dynamic response is generated by simulating three-phase fault at bus 3 on the line from bus 3 to bus 101 . The fault starts at 0.1 seconds and clears at 0.15 seconds. The dynamic response for the case before and after MANGO adjustment is shown in Figure 22. The blue line shows that responses from the original base case. The red dashed line shows 
the response of system after implementing MANGO recommendation. It can be observed that the oscillation response damped out more quickly with the system after MANGO adjustment. The figure clearly shows the damping improvement after the implementation of the MANGO recommendation. Prony analysis on the simulated ringdown signal confirms the damping improvement.

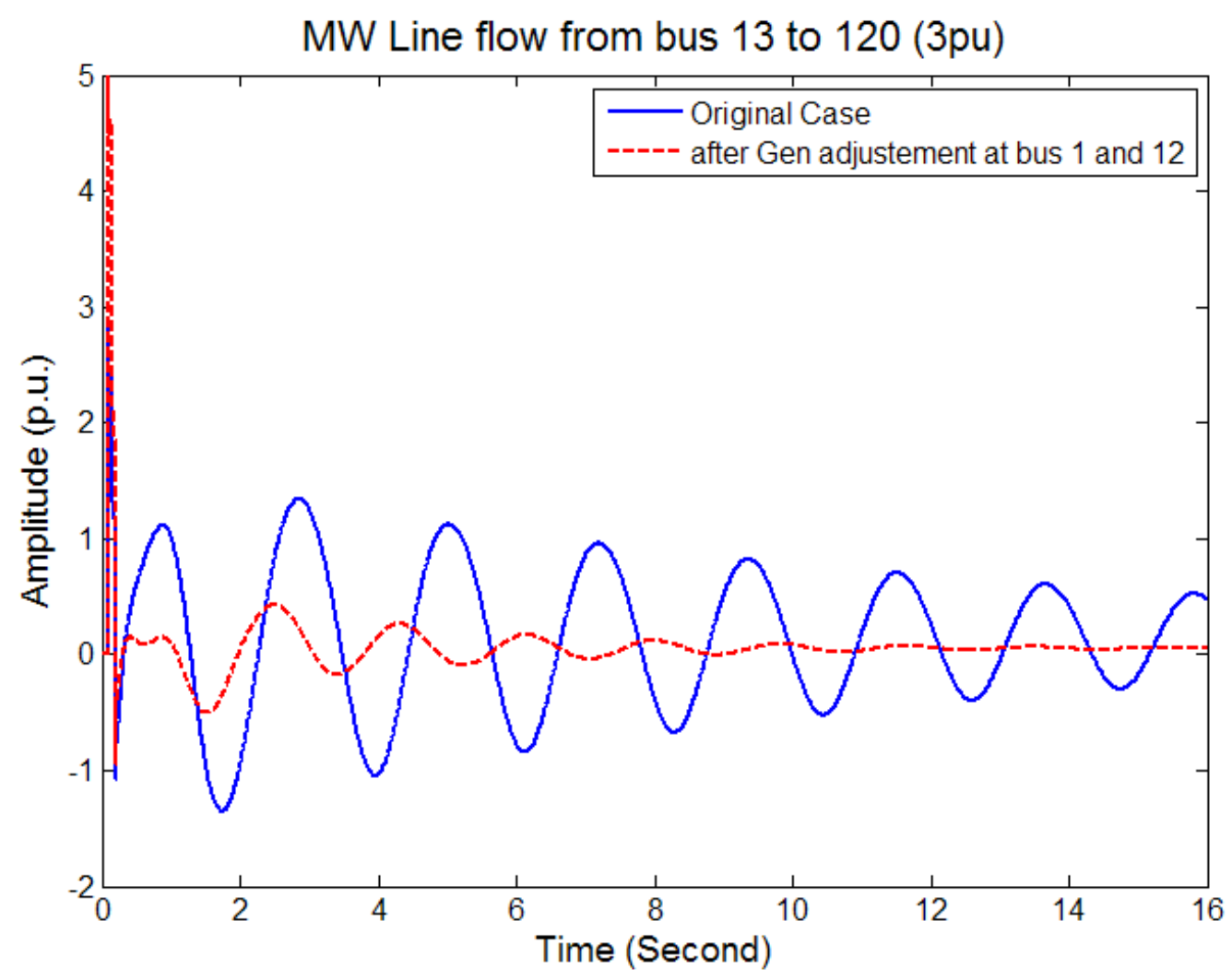

Figure 22. Effectiveness of MANGO recommended adjustments for two-area, four-machine system

\subsubsection{Case Studies Using the 34-machine Model}

To evaluate the performance of the proposed algorithm for a medium-size system, 34-machine system model, shown in Figure 17, is used to generate simulation data. For the base case, the system has an interarea mode at Freq $=0.339 \mathrm{~Hz} \mathrm{~Hz}$ and $\mathrm{DR}=1.26 \%$, which is chosen as the mode of interest. To simulate the random load and generation variation, random perturbations are added to each generator and load to generate 1000 cases. Similar to the previous subsection, two levels (i.e., $1 \%$ and $10 \%$ ) of perturbation are simulated. The Power System Toolbox is again used for simulating and calculating the eigenvalues for the two perturbation levels. The modal sensitivities calculated directly from the model are used as reference for evaluating the performance of the proposed algorithm. To unify the signs of loads and generations, the loads are again denoted as negative generation. The study results for damping ratio sensitivity analysis are summarized in Figure 23. The sensitivity is relative to the reference generator Gen1. It can be observed that the estimation results are consistent with direct computation. This consistency verifies the validity of the proposed algorithm.

To apply the analysis results shown in Figure 23 for generating MANGO recommendation, the largest sensitivity difference is examined when identifying the most effective generator pair. Figure 23 shows 
that the most effective pair is generators at buses 118 and 76 with a relative sensitivity of about $0.8 \% / p$.u. The corresponding MANGO recommendation is "increase the generator power output at bus 76 (Gen 32) by 3 p.u. and decrease generator at bus 118 (Gen 34) by 3 p.u., and the damping ratio is expected to increase by about $2.4 \%$ (3 p.u. * $0.8 \%$ /p.u.)". The damping improvement is confirmed by the eigenvalue analysis shown in Figure 24, where the two estimates (shown in black and green) are very close to the reference sensitivity (shown in red).

To further verify this MANGO recommendation, a time domain dynamic response is generated by simulating three-phase fault at bus 86 on the line from bus 86 to bus 87 . The fault starts at 5 seconds and clears at 5.095 seconds. The dynamic response for cases before and after MANGO adjustment is shown in Figure 25. The blue line shows that responses from the original base case. The red dashed line shows the response of system after implementing MANGO recommendation. It can be observed that the oscillation response damped out more quickly with the system after MANGO adjustment. The figure clearly shows the damping improvement after the implementation of the MANGO recommendation.

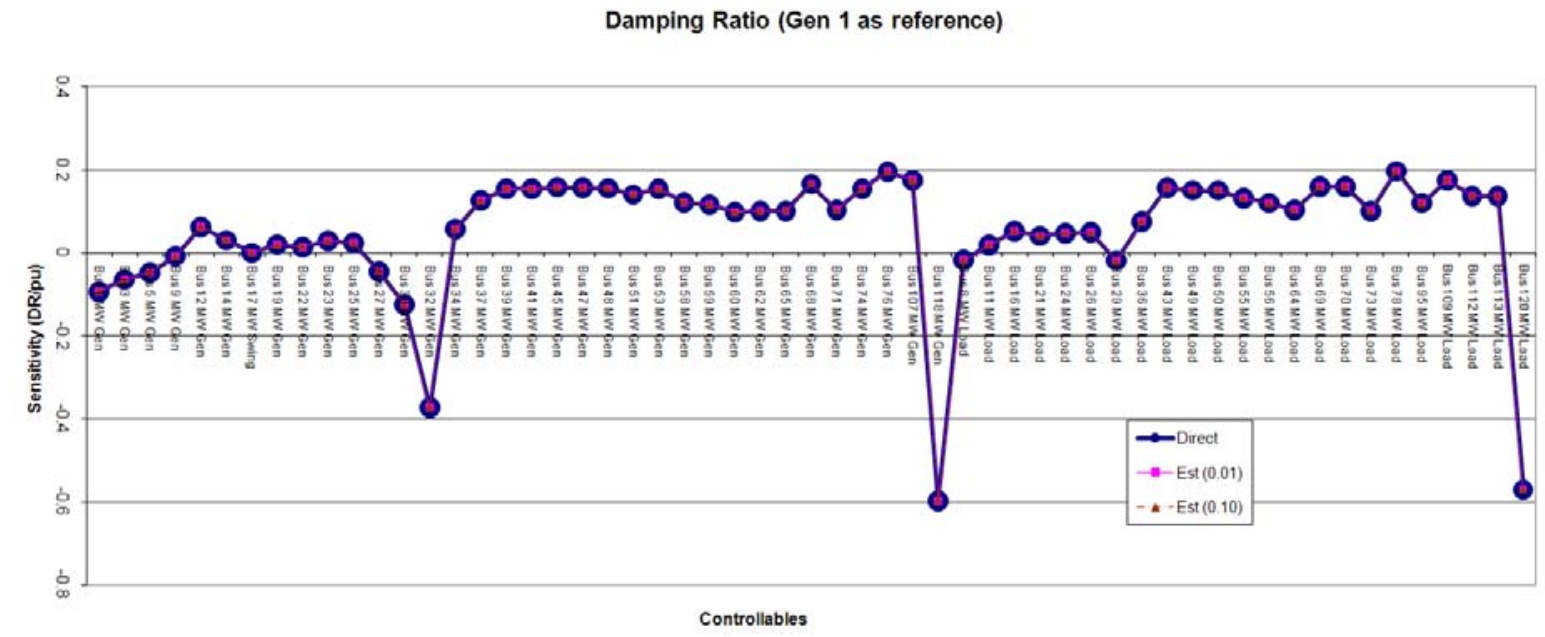

Figure 23. Estimated modal sensitivity of the 34-machine model 


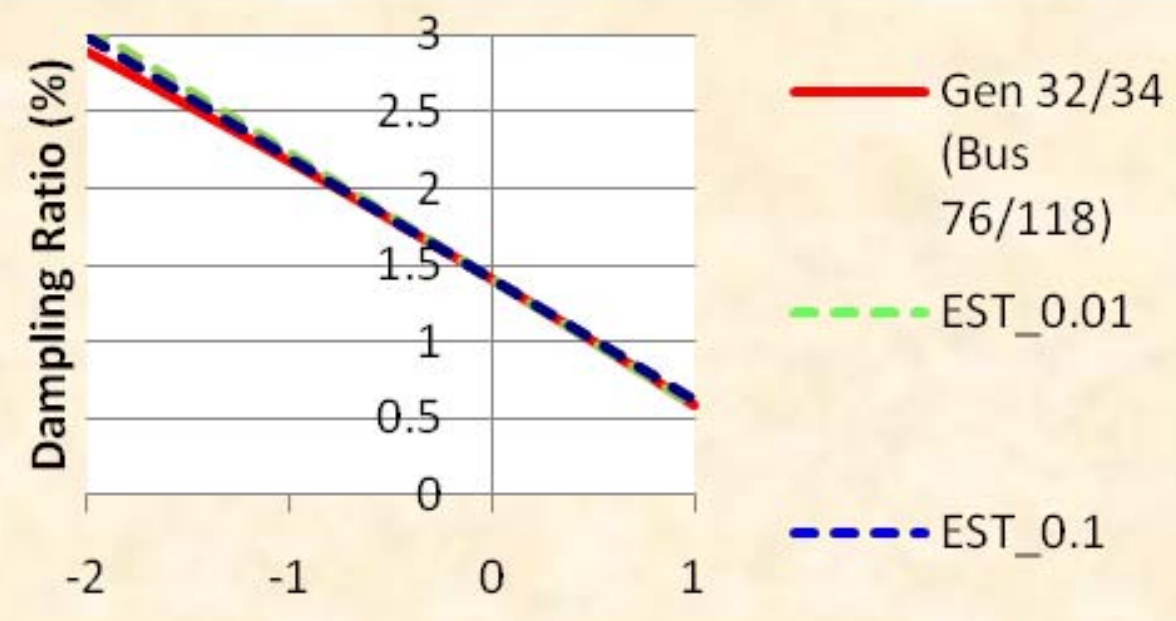

\section{Real Power Injection at Generator Pairs (pu)}

Figure 24. Effectiveness of MANGO recommended adjustment for 34-machine model

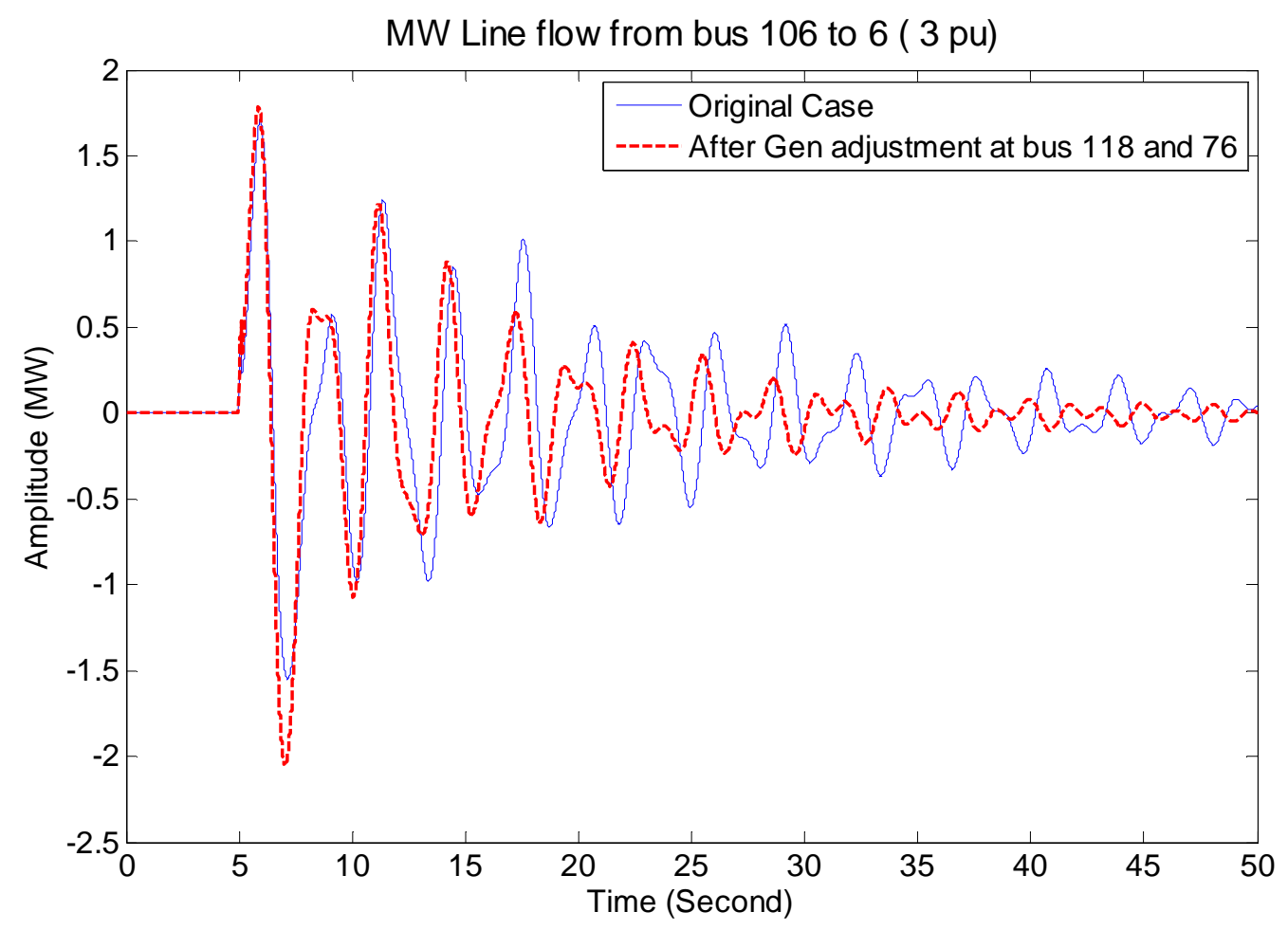

Figure 25. Effectiveness of MANGO recommended adjustment for the 34 machine model

\subsection{Relative Modal Sensitivity Estimation with respect to Tie Line Flows}

The appearance of lightly damped modes has been associated with "transmitting bulk power over long distances" [27]. The small signal stability problem puts an upper limit on how much power can be 
transferred over some tie lines. BPA DSO 303 provides guidelines to reduce tie line flows when light damping modes are observed. It is desirable for grid operation to find the modal sensitivity with respect to the power flow of key transmission lines. In this subsection, an iterative method is formulated for estimating the modal sensitivity with respect to power flow on major transmission lines. The estimation process uses a series of ModeMeter results. The algorithm takes advantage of the natural variations in oscillation modes and operating points over a period of time. Preliminary tests have been performed with the two-area-four-machine system and the 34-machine model mentioned in the previous subsection. The performance of the proposed algorithms is evaluated based on simulation data from the tests.

\subsubsection{Algorithm Description}

Similar to the modal sensitivity with respect to generation and load, when the operational points of power grid do not change significantly, the relationship between the modes changes and line flow changes can be described as

$$
\Delta \lambda^{(i)}=\frac{\partial \lambda}{\partial p_{1}} \Delta p_{1}^{(i)}+\frac{\partial \lambda}{\partial p_{2}} \Delta p_{2}^{(i)}+\ldots+\frac{\partial \lambda}{\partial p_{\text {NLine }}} \Delta p_{\text {NLine }}^{(i)}+\varepsilon^{(i)}
$$

where the $\Delta p$ represents real power changes in the transmission line from previous instance, NLine represents the total number of transmission lines, the superscript $(i)$ denotes that it is the $i$ th perturbation, $\lambda$ represents the eigenvalue of interest, and $\Delta \lambda$ denotes the changes of the eigenvalue from previous instance. Note that for real-time application, the modal sensitivity $\partial \lambda / \partial p_{j}$ 's are unknown and need to be estimated. The $\Delta \lambda$ can again be estimated from real-time measurement using a ModeMeter algorithm. $\Delta p$ can be derived from field measurement. To estimate the modal sensitivity with respect to line flows, the following equations can be formed after $N$ time instances.

$$
\left[\begin{array}{c}
\Delta \lambda^{(1)} \\
\Delta \lambda^{(2)} \\
\vdots \\
\Delta \lambda^{(N)}
\end{array}\right]=\left[\begin{array}{cccc}
\Delta p_{1}^{(1)} & \Delta p_{2}{ }^{(1)} & \cdots & \Delta p_{\text {NLine }}^{(1)} \\
\Delta p_{1}^{(2)} & \Delta p_{2}{ }^{(2)} & \cdots & \Delta p_{\text {NLine }}^{(2)} \\
\vdots & \vdots & \ddots & \vdots \\
\Delta p_{1}^{(N)} & \Delta p_{2}{ }^{(N)} & \cdots & \Delta p_{\text {NLine }}^{(N)}
\end{array}\right]\left[\begin{array}{c}
\frac{\partial \lambda}{\partial p_{1}} \\
\frac{\partial \lambda}{\partial p_{2}} \\
\vdots \\
\frac{\partial \lambda}{\partial p_{\text {NLine }}}
\end{array}\right]+\left[\begin{array}{c}
\varepsilon^{(1)} \\
\varepsilon^{(2)} \\
\vdots \\
\varepsilon^{(N)}
\end{array}\right]
$$

To simplify notation, Equation (21) can be written in matrix format as

$$
\Delta \lambda=\Delta p \cdot \frac{\partial \lambda}{\Delta p}+\varepsilon
$$

When $N \geq N L i n e$, Equation (21) may be solved for the modal sensitivity using a least-squares optimization. However, because of correlations among the real power flows on transmission lines, the columns in $\Delta p$ matrix of Equation (22) can be linearly dependent. In this case, the $\Delta p$ matrix is ill-conditioned. Standard least-square solvers usually fail to provide a good solution for ill-conditioned problems.

The linear dependency of columns in the $\Delta p$ matrix of Equation (21) is much more complex than that of the $\Delta \mathrm{p}$ matrix in Equation (17). For example, in $\Delta p$ the only constraint is Equation (18). There can be $N G e n+N$ Load-1 columns of data in $\Delta \mathrm{p}$ that are linearly independent. In comparison, the linear 
dependency of $\Delta \mathrm{p}$ matrix can take many forms. For example, in Figure 12 of the two-area-four-machine model, there are two transmission lines between bus 3 and bus 101. Because of the symmetric structure, the power flow in those two transmission lines are highly correlated. Another example is that the sum of real power flow into a bus equals the sum of the real power flow out of the bus. In Figure 12, the sum of real power flows into bus 3 equals to 0 . These relationships determine that the columns of $\Delta p$ matrix in Equation (22) are highly correlated. Consequently, the Equation (22) is an ill-conditioned problem. Because of the complexity of the linear dependency, it is not possible to resolve the ill-conditioned problem analytically as was done for the case of generation and load sensitivities in Section 5.2. Therefore, a new numerical method is proposed in this study.

Several commonly used algorithms for solving ill-conditioned regression problem are explored. Some initial tests were performed and revealed that these algorithms cannot be applied directly to solve the problem defined by Equation (21). A brief discussion and summary of these algorithms is as follows:

1. Standard regression method: In the statistics toolbox of MATLAB ${ }^{\circledR}$, the standard linear regression is provided by function [regress]. To deal with linearly dependent columns, [regress] generates a 0 solution for the linearly dependent column. Applying the function [regress] directly on the simulation data generated from the two-area-four-machine model, big errors in sensitivity estimation are observed. Instead of perfectly linearly dependant, most of columns of the $\Delta p$ matrix are very close to be linearly dependent. This causes the ill-conditioned problem and results in significant errors in the solution. Therefore, the standard regression method is not suitable for solving the modal sensitivity problem.

2. Partial least-square regression: In the statistics toolbox of MATLAB ${ }^{\circledR}$, the partial linear regression is provided by function [plsregress]. To find the fundamental relationship between the line flow and the mode, latent variables are introduced by the partial least square regression. The latent variables are independent variables, which serve as a transform to connect transmission line flows and oscillatory modes. The ill-conditioned problem is well taken care of by this method. Yet, the latent variables are formed according to mathematical needs to overcome the illconditioned problem. The latent variables do not carry any physical meaning and, in turn, cannot provide the guidance needed for MANGO recommendations. The modal sensitivity cannot be readily obtained from the solution of partial least square regression.

3. Stepwise regression method: In the statistics toolbox of MATLAB ${ }^{\circ}$, the stepwise regression is provided by function [stepwisefit]. "Stepwise regression is a systematic method for adding and removing terms from a multilinear model based on their statistical significance in a regression" [43]. It uses statistical methods to remove the redundant variables. This method appears to be a good fit for the modal sensitivity problem. However, a detailed review shows that the stepwise regression method checks the null hypothesis (i.e., whether a coefficient should be set to 0 ) to determine whether a variable should be included. According to initial tests on the two-area-fourmachine system model, this results in some lines with little changes being mistakenly included with top priority, and the inclusion of these variables results in large errors in sensitivity estimation.

The proposed algorithm for the modal sensitivity analysis adopts a similar estimation procedure as the stepwise regression algorithms. The major change is that instead of focusing on the null hypothesis tests, the proposed method is focused on the percentage of the contribution from the explaining variables. In the 
line flow case, the explaining variables are transmission line flows. The proposed algorithm is referred to as a modified stepwise regression (MSR). The essence of the MSR is to select a subset of independent columns from matrix $\Delta \mathrm{p}$. This subset of independent columns should be able to reflect most of the variations of the modes. The modified stepwise regression is carried out using the following procedure:

1. Set $[n$ Step $]=1$; Initialize the subset of selected independent columns to be empty ; Initialize the subset of processed columns to be empty; Initialize the subset of remaining columns to have all the columns from matrix $\Delta \mathrm{p}$.

2. Add one column from the remaining columns to the selected independent columns one at a time to form a group of candidate subsets with [nStep] columns. Fit each candidate subset to the mode changes using standard linear regression function [regress].

3. Identify one candidate subset, which can best explain the mode changes. The corresponding column added in step (2) from remaining columns is moved permanently to the subset of selected independent columns.

4. Fit each of the remaining columns with the selected independent columns to identify the linear dependency. If a column is identified to be linearly dependent on the selected independent columns, it is moved to the subset of processed columns. The correlation between the processed columns and selected independent column are recorded.

5. Set $[n$ Step $]=[n$ Step $]+1$.

6. If the subset of remaining columns becomes empty or adding an additional column in the selected independent columns does not help improve the mode change significantly, end the procedure, otherwise go to step 2.

With the MSR algorithm, a subset of independent columns in matrix $\Delta p$ can be identified. This subset of the independent columns can be used to fit the mode changes without causing an ill-conditioned problem. Also, the line flows can be sorted according to their contribution to explaining the mode changes. In addition, correlation between the processed column and selected independent columns can be used to group the transmission line together to reveal their dependency.

\subsubsection{Case Studies Using a Two-Area Four-Machine System}

To verify the performance of the proposed MSR method, the two-area four-machine system shown in Figure 12 is used to generate simulation data for studying the modified algorithm in estimating modal sensitivities. The study was again conducted with the Power System Toolbox, version 3.0. For the base case, the system has an inter-area mode at Freq $=0.65 \mathrm{~Hz}$ and $\mathrm{DR}=5.37 \%$, which is chosen as the mode of interest. To simulate the random generation variation, $10 \%$ random perturbations are added to the generators to create 300 cases. To simplify the study, loads are kept unchanged. The study results for damping ratio sensitivity using the MSR algorithm are summarized in Figure 26 and Table 6. 


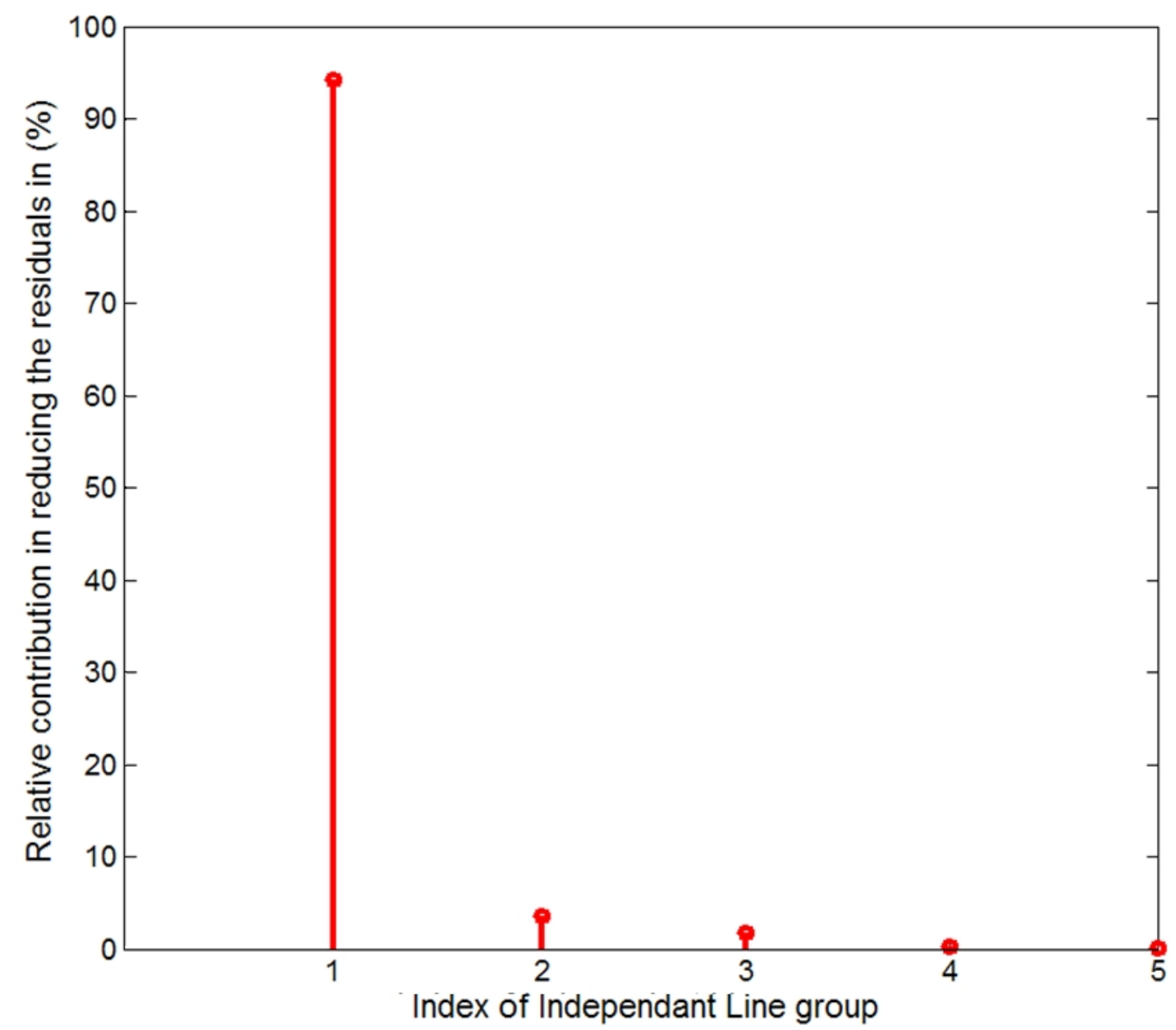

Figure 26. Percentage of Contributions from line flow to the damping ratio in the two-area, four-machine system

Table 6. Mode Sensitivity with Respect to Line Flow in the Two-Area, Four-Machine System

\begin{tabular}{|c|c|c|c|c|c|}
\hline Group Index & Line Index & From Bus & To Bus & $\begin{array}{l}\text { Mode Sensitivity } \\
\text { of DR changes. }\end{array}$ & $\begin{array}{c}\text { Percentage } \\
\text { Contribution to } \\
\text { mode DR }\end{array}$ \\
\hline \multirow{6}{*}{1} & 5 & 3 & 101 & -2.38 & $94.2 \%$ \\
\hline & 4 & 3 & 20 & - & - \\
\hline & 6 & 3 & 101 & - & - \\
\hline & 11 & 13 & 101 & - & - \\
\hline & 12 & 13 & 101 & - & - \\
\hline & 13 & 13 & 120 & - & - \\
\hline \multirow{3}{*}{2} & 1 & 1 & 10 & -0.22 & $3.5 \%$ \\
\hline & 2 & 2 & 20 & - & - \\
\hline & 7 & 10 & 20 & - & - \\
\hline 3 & 3 & 3 & 4 & $5.6 \mathrm{e} 8$ & $1.7 \%$ \\
\hline 4 & 4 & 13 & 14 & $-3.8 \mathrm{e} 5$ & $0.2 \%$ \\
\hline \multirow{3}{*}{5} & 9 & 12 & 120 & -0.12 & $0.0 \%$ \\
\hline & 8 & 11 & 110 & - & - \\
\hline & 14 & 110 & 120 & - & - \\
\hline
\end{tabular}

Note: "-" indicates that this line is not selected in the dependence analysis using the MSR algorithm. 
In Table 6, columns of $\Delta \mathrm{p}$ are arranged into five groups. The first transmission line in each group consists of the selected independent columns of $\Delta \mathrm{p}$. The other transmission lines in the same group are highly correlated to the first line. It can be observed from Figure 26 that about $94 \%$ of mode DR changes can be explained by the power flow on the transmission lines in group \#1. This means that the mode DR is most sensitive to the power flow in group \#1. The transmission lines in group \#1 are marked in magenta lines in Figure 27. It can be observed that these are inter-area tie lines and lines highly correlated to tie lines. The mode sensitivity with respect to the tie line, defined by bus 3 to 101, is -2.38 . This means that to increase the DR of the inter-area mode by $2.38 \%$, the real power flow in the tie line (from 3 to 101 ) should decrease by $100 \mathrm{MW}$.

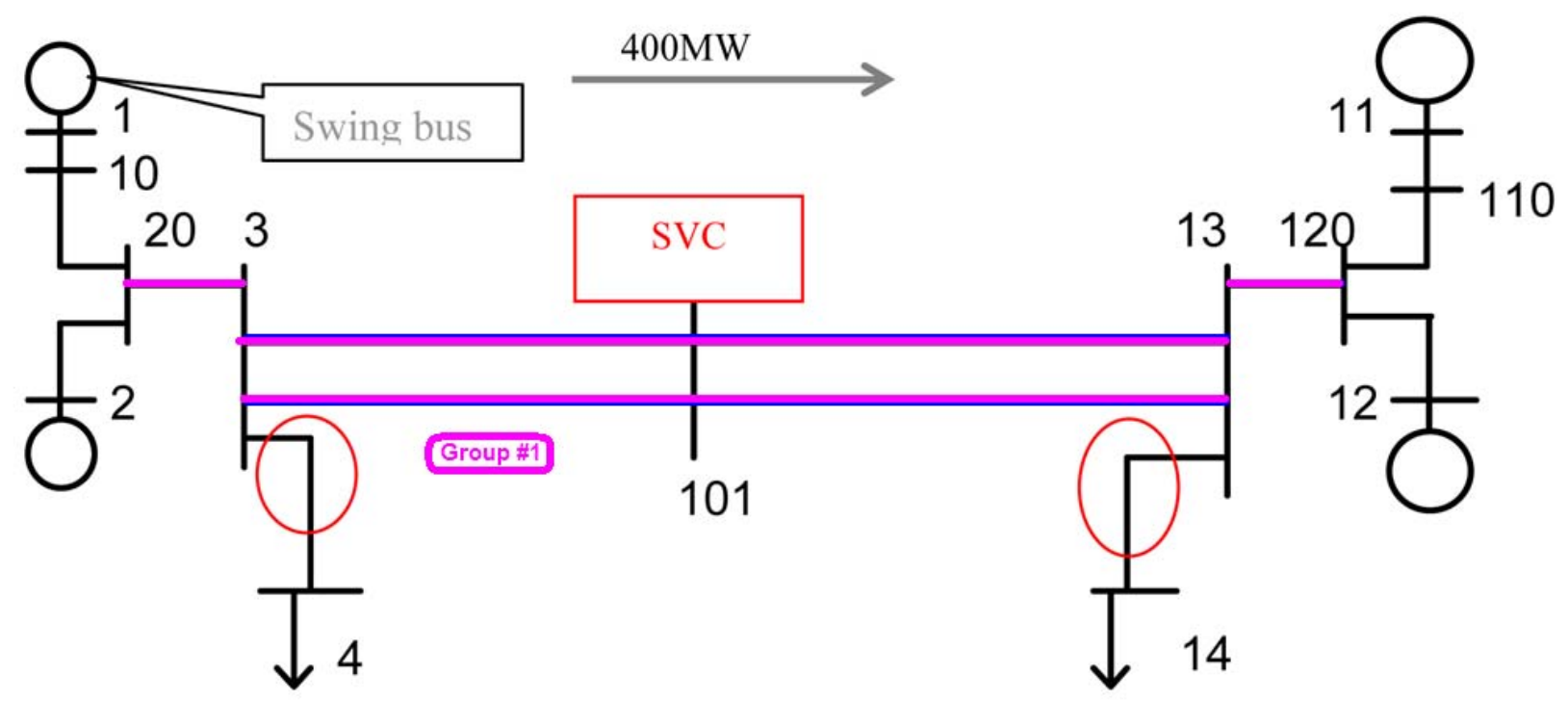

Figure 27. Transmission line with major modal sensitivities in the two-area, four-machine system [42]

It is also observed that the sensitivity with respect to lines \#3 and \#4 is extremely large, while the percentages of contribution are small. Further examination of the data reveals that those lines do not have significant power flow changes. The sensitivity solution for these lines should be considered invalid.

With the sensitivity derived, it can be observed that the tie line plays a dominant role in the inter-area mode.

The DR changes of the inter-area mode are related to the line flow as follows:

$$
\Delta D R=-2.38 \Delta p_{3->101}-0.22 \Delta p_{1->10}+\varepsilon
$$

To verify the results, some additional independent validation cases are generated. The DR changes predicted from Equation (25) are compared against the true mode changes from PST simulation. The results are summarized in the Table 7 . It can be observed from the Table 7 that the estimation results are consistent with the true sensitivities, with only minor estimation errors. 
Table 7. Validation of Mode Sensitivities with Respect to Line Flow in the Two-Area, Four-Machine System

\begin{tabular}{|c|c|r|r|r|r|r|r|}
\hline $\begin{array}{c}\text { Ref } \\
\text { bus }\end{array}$ & Adjust Gen & $\begin{array}{c}\text { Adjustment } \\
\text { (PU) }\end{array}$ & $\Delta p_{3->101}$ & $\Delta p_{1->10}$ & $\begin{array}{c}\text { Estimated } \\
\text { DR changes } \\
\text { (\%) }\end{array}$ & $\begin{array}{c}\text { True DR } \\
\text { changes } \\
\text { (\%) }\end{array}$ & Relative Err \\
\hline 1 & Gen3 (on bus 11) & 0.5 & -0.254 & -0.546 & $0.724 \%$ & $0.790 \%$ & $-8.4 \%$ \\
\hline 1 & Gen3 (on bus 11) & 1.0 & -0.502 & -1.076 & $1.432 \%$ & $1.475 \%$ & $-2.9 \%$ \\
\hline 1 & Gen4 (on bus 12) & 0.5 & -0.265 & -0.569 & $0.756 \%$ & $0.763 \%$ & $-1.0 \%$ \\
\hline 1 & Gen 4 (on bus 12) & 1.0 & -0.525 & -1.125 & $1.498 \%$ & $1.440 \%$ & $4.0 \%$ \\
\hline
\end{tabular}

\subsubsection{Case Studies Using the 34-machine Model}

A medium-size system, the 34-machine system model, shown in Figure 17, is used to further evaluate the performance of the proposed algorithm. For the base case, the system has an interarea mode at Freq $=0.339 \mathrm{~Hz}$ and $\mathrm{DR}=1.26 \%$, which is chosen as the mode of interest. To simulate the random generation variation, $10 \%$ random perturbations are added to each generator to generate 1000 cases. To simplify the study, loads are again kept unchanged. The study results for damping ratio sensitivity using the MSR algorithm are summarized in Table 8 and Figure 28. For clarity, only the lines with a contribution higher than $0.5 \%$ are presented.

In Table 8, major columns of $\Delta \mathrm{p}$ are arranged in 14 groups. The first transmission line in each group consists of the selected independent columns of $\Delta \mathrm{p}$. The other transmission lines in the same group are highly correlated to the first line. It can be observed from Figure 28 that two groups of transmission lines contribute significantly to the variation of the inter-area mode. The first group contributes about $39.4 \%$ of the DR changes. The location of the first line group is marked in blue in Figure 29. Also overlaid on this map are the mode shapes of the inter-area mode. It can be observed that the first line group is a group of tie lines between two oscillation areas defined by the mode shape. The sensitivity of first line is -0.53 , which means that the DR of the inter-area mode can be increased by about $0.53 \%$ if the line flow from bus 83 to bus 103 is reduced by $100 \mathrm{MW}$. The second group of tie lines is located at the upper position and marked in magenta in Figure 29. Its contribution to the DR variation of the inter-area mode is about $35.4 \%$. According to the mode shape, these are also tie lines between the two areas which oscillate against each other. The sensitivity is 1.08 , which means that the DR of the inter-area mode can be increased by $1.08 \%$ if the tie line flow from bus 119 to bus 117 is reduced by $100 \mathrm{MW}$. 
Table 8. Modal Sensitivity with Respect to Line Flow for the 34-machine Model

\begin{tabular}{|c|c|c|c|c|c|}
\hline Group Index & Line Index & From Bus & To Bus & $\begin{array}{l}\text { Mode Sensitivity } \\
\text { of DR changes. }\end{array}$ & $\begin{array}{c}\text { Percentage } \\
\text { Contribution to } \\
\text { mode DR }\end{array}$ \\
\hline \multirow{2}{*}{1} & 97 & 83 & 103 & -0.53 & $39.4 \%$ \\
\hline & 98 & 103 & 102 & - & - \\
\hline \multirow{4}{*}{2} & 166 & 117 & 119 & 1.08 & $35.4 \%$ \\
\hline & 163 & 6 & 106 & - & - \\
\hline & 164 & 106 & 119 & - & - \\
\hline & 165 & 106 & 117 & - & - \\
\hline 3 & 79 & 84 & 20 & -0.22 & $4.8 \%$ \\
\hline 4 & 133 & 49 & 113 & 0.05 & $2.3 \%$ \\
\hline \multirow{6}{*}{5} & 73 & 33 & 83 & -0.50 & $3.8 \%$ \\
\hline & 14 & 32 & 33 & - & - \\
\hline & 74 & 33 & 85 & - & - \\
\hline & 75 & 83 & 85 & - & - \\
\hline & 76 & 83 & 84 & - & - \\
\hline & 77 & 83 & 84 & - & - \\
\hline 6 & 65 & 7 & 10 & -0.03 & $2.8 \%$ \\
\hline \multirow{3}{*}{7} & 142 & 57 & 66 & 0.13 & $1.4 \%$ \\
\hline & 143 & 57 & 98 & - & - \\
\hline & 144 & 98 & 66 & - & - \\
\hline 8 & 69 & 10 & 13 & -0.06 & $0.71 \%$ \\
\hline 9 & 161 & 72 & 69 & -0.06 & $0.73 \%$ \\
\hline 10 & 89 & 86 & 88 & -0.32 & $1.07 \%$ \\
\hline \multirow{3}{*}{11} & 58 & 80 & 7 & -0.28 & $1.02 \%$ \\
\hline & 56 & 80 & 81 & - & - \\
\hline & 57 & 81 & 7 & - & - \\
\hline 12 & 124 & 114 & 46 & 0.08 & $0.80 \%$ \\
\hline \multirow{6}{*}{13} & 138 & 113 & 57 & 0.09 & $0.68 \%$ \\
\hline & 136 & 113 & 57 & - & - \\
\hline & 137 & 113 & 57 & - & - \\
\hline & 138 & 113 & 57 & - & - \\
\hline & 139 & 113 & 116 & - & - \\
\hline & 140 & 116 & 57 & - & - \\
\hline 14 & 40 & 29 & 28 & 0.03 & $0.51 \%$ \\
\hline
\end{tabular}

Note: "-" indicates that this line is not selected in the dependence analysis using the MSR algorithm. 


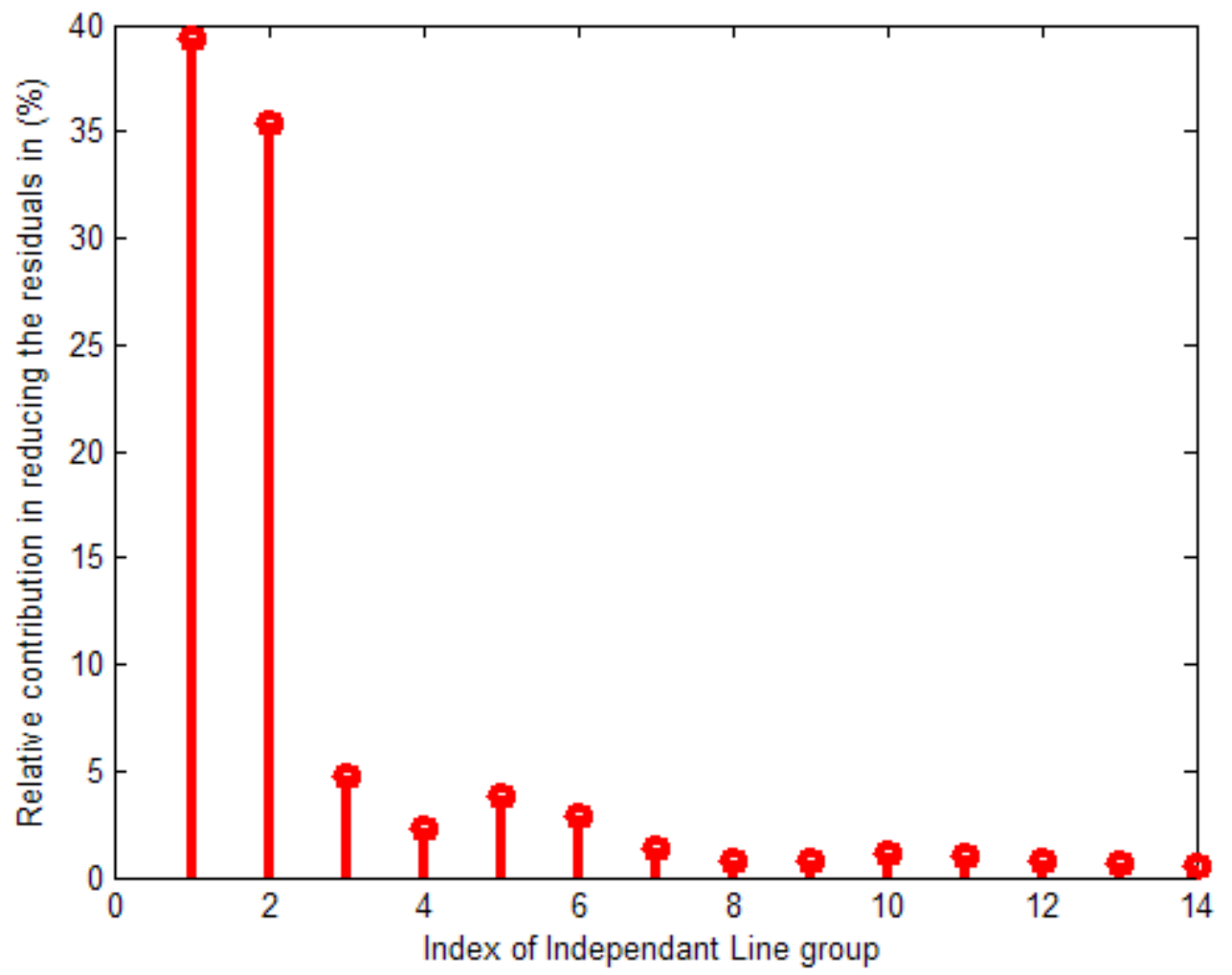

Figure 28. Percentage of contributions from line flow to the damping ratio in the 34-machine model 


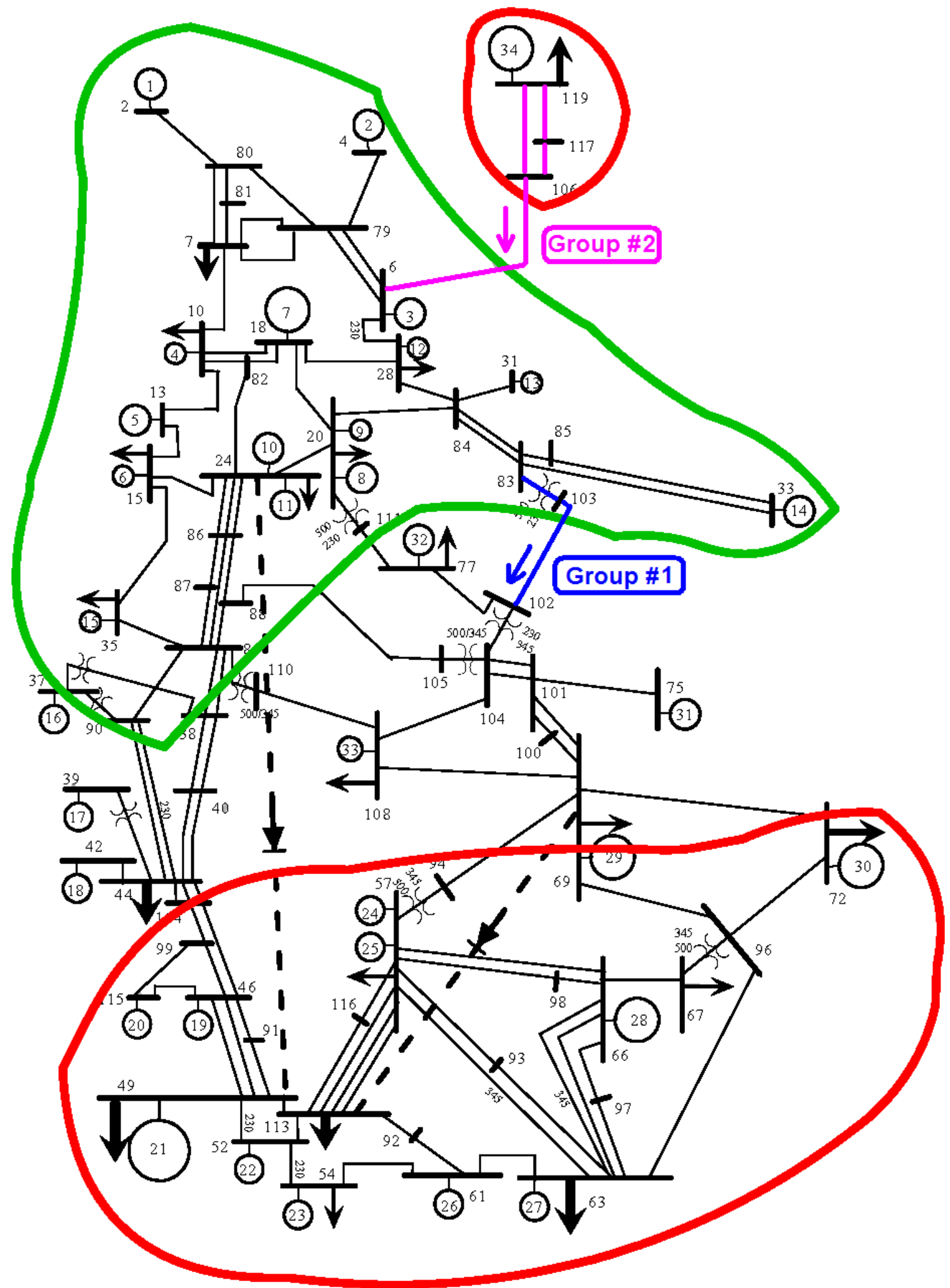

Figure 29. Transmission lines with major modal sensitivities in the 34-machine model 


\subsection{MANGO Method 2: Nonlinear Mapping Based on Artificial Neural Network}

The nonlinear relationship between modal damping and operating parameters such as generator power settings can be described by a nonlinear multiple-input-multiple-output (MIMO) function. The inputs are the operating parameters and the outputs are the oscillation modes of interest. Once such a function is identified, it can be used to derive modal sensitivity information. To approximate this nonlinear function, an artificial neural network (ANN) approach is proposed for the MANGO procedure. "Neural networks are good at fitting functions and recognizing patterns. In fact, there is proof that a fairly simple neural network can fit any practical function [44]." A three-layer feed-forward ANN network is used, consisting of the input, middle and output layers. The Levenberg-Marquardt method [43] is used to tune the parameters in the ANN network. There are three steps in applying ANN for generating MANGO recommendations:

1. Collect data for training, validation and testing. For each data set, the input variables are the operator controllable variables, e.g., the output power of a generator, adjustable load devices, etc. The output variables are the modes of interest. Data can be collected from simulation cases and actual operating cases. Each case is a set of inputs and outputs. It is desirable that the data sets cover the normal operational region so that interpolation (instead of extrapolation) can be used to reduce errors. Also, it is desirable to have limited number of inputs to avoid the "curse of dimensionality" problem during the ANN training procedure. Thus, a subset of operator controllable variables is selected based on eigenvalue analysis results such as mode shapes and participation factors, as well as operating experience. Only the influential input variables are included in the selected subset of input variables for building ANN models.

2. Train, validate and test the ANN. The collected data are divided into three sets (i.e., training sets, validation sets, and test sets) to create an ANN. The training set is used to tune the parameters of ANN. The validation data set is used to check if the training should continue or stop. The training procedure should stop when further training cannot further reduce the mismatches in the validation data set. The test set is used to independently check the accuracy of the trained ANN. If an ANN cannot reach a desired level of accuracy for the test sets, the model order may need to be adjusted and the ANN needs to be re-trained.

3. Use the trained ANN to derive modal sensitivity. Real-time measurement and ModeMeter information are used at this step. The fundamental idea is to iteratively identify the most effective adjustment to reach a targeted damping level. At each iteration step, a small perturbation is introduced to each of the input variables in the ANN. The deviation of modes is calculated and used as modal sensitivity with respect to the perturbed input variable. An adjustment is applied to the input variables identified by the largest sensitivities. The iterative approach is to ensure that the nonlinearity is considered in the process. After all the steps, the final adjustment is determined.

This ANN-based approach is applied to the 34-machine model, which contains 34 generators and 122 buses, while also retaining most of the full WECC system's properties of small signal stability. An interarea mode at about $0.4 \mathrm{~Hz}$ is studied, and all the generator power outputs and load consumptions are selected as input variables. Data sets (of size 9000) are generated for training and testing by randomly 
adjusting the power flow conditions. Training accuracy is promising, as indicated in Figure 30 by the small mismatch between the ANN-predicted results and the testing data sets.

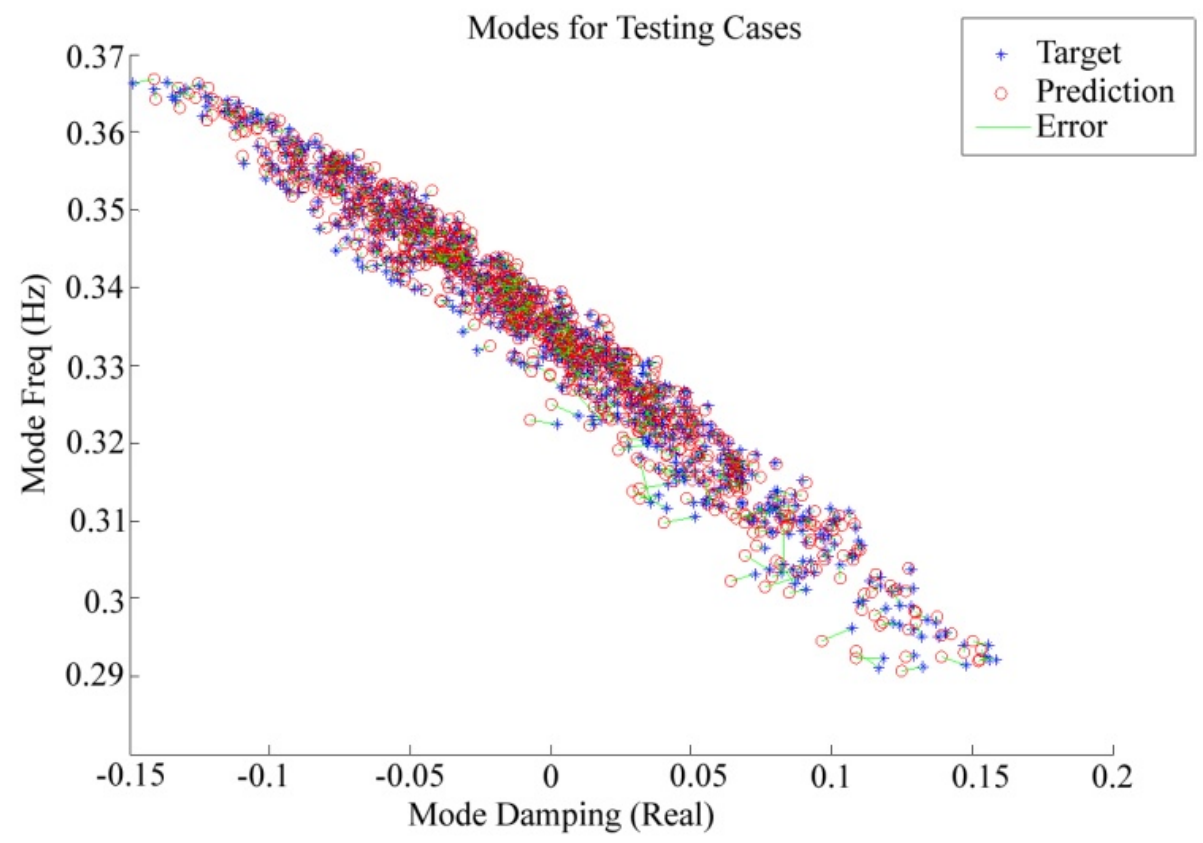

Figure 30. Training and testing results of the ANN for the $0.4 \mathrm{~Hz}$ mode

Figure 31 shows the front panel of a MATLAB-based graphical user interface (GUI) developed to test the ANN-based MANGO approach.

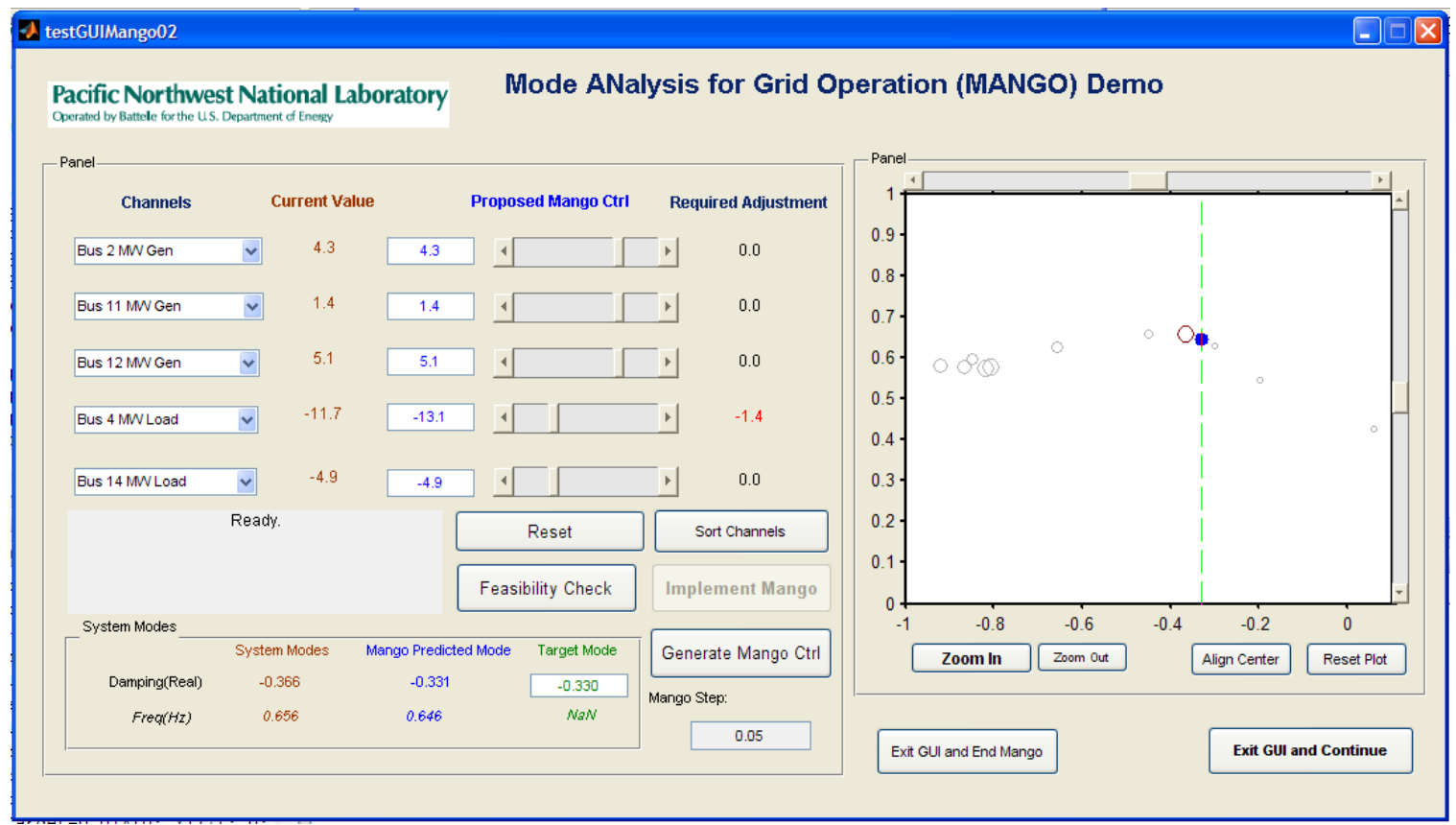

Figure 31. MATLAB GUI for testing the proposed ANN-based MANGO approach 
The ANN model is used to generate MANGO control to increase the damping of the inter-area mode in the 34-machine model (Figure 17). For the base case, the inter-area mode is lightly damped, with damping ratio $=1.26 \%$ and frequency $=0.339 \mathrm{~Hz}$. A goal was established to improve inter-area mode's damping ratio to $6.41 \%$. According to the ANN model, the recommendation of MANGO controls are generated and shown in Figure 32. As Figure 32 shows, the MANGO controls incorporate both generation changes and load changes. In a practical implementation, these changes would likely be accomplished via load control or shedding (via direct control or demand response-type incentives) and generator re-dispatch. The location of top five MANGO controls are shown in Figure 33. As can be observed, the MANGO control, in general, reduces the power flow from the upper portion of the system to the lower portion. To verify this MANGO control strategy, the recommended controls are implemented with PST. The resulting inter-area mode is changed to damping ratio $=7.36 \%$ and frequency $=0.375 \mathrm{~Hz}$. The MANGO control results exceed the initial goal.

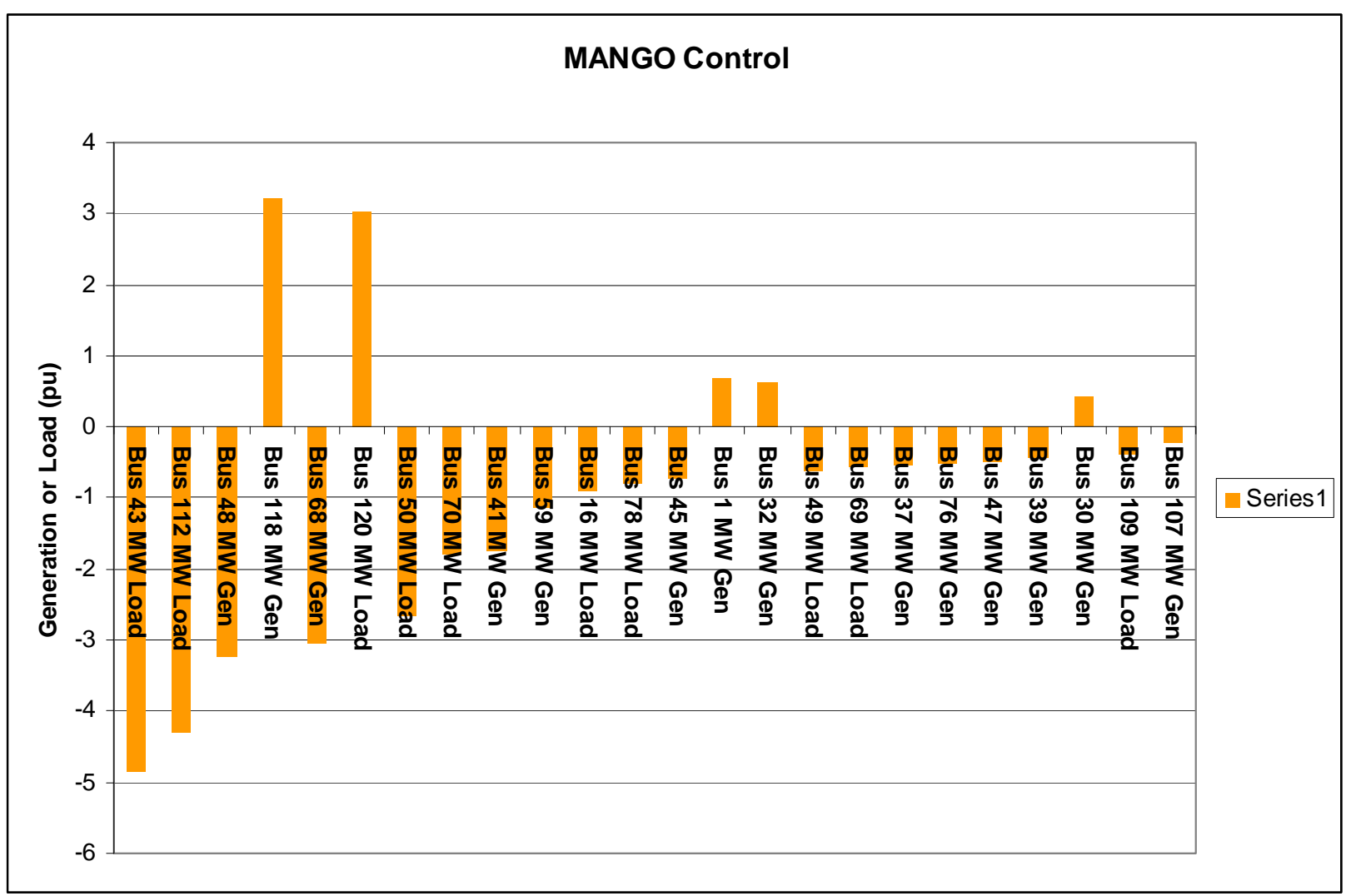

Figure 32. MANGO control for improving the Alberta mode damping. 


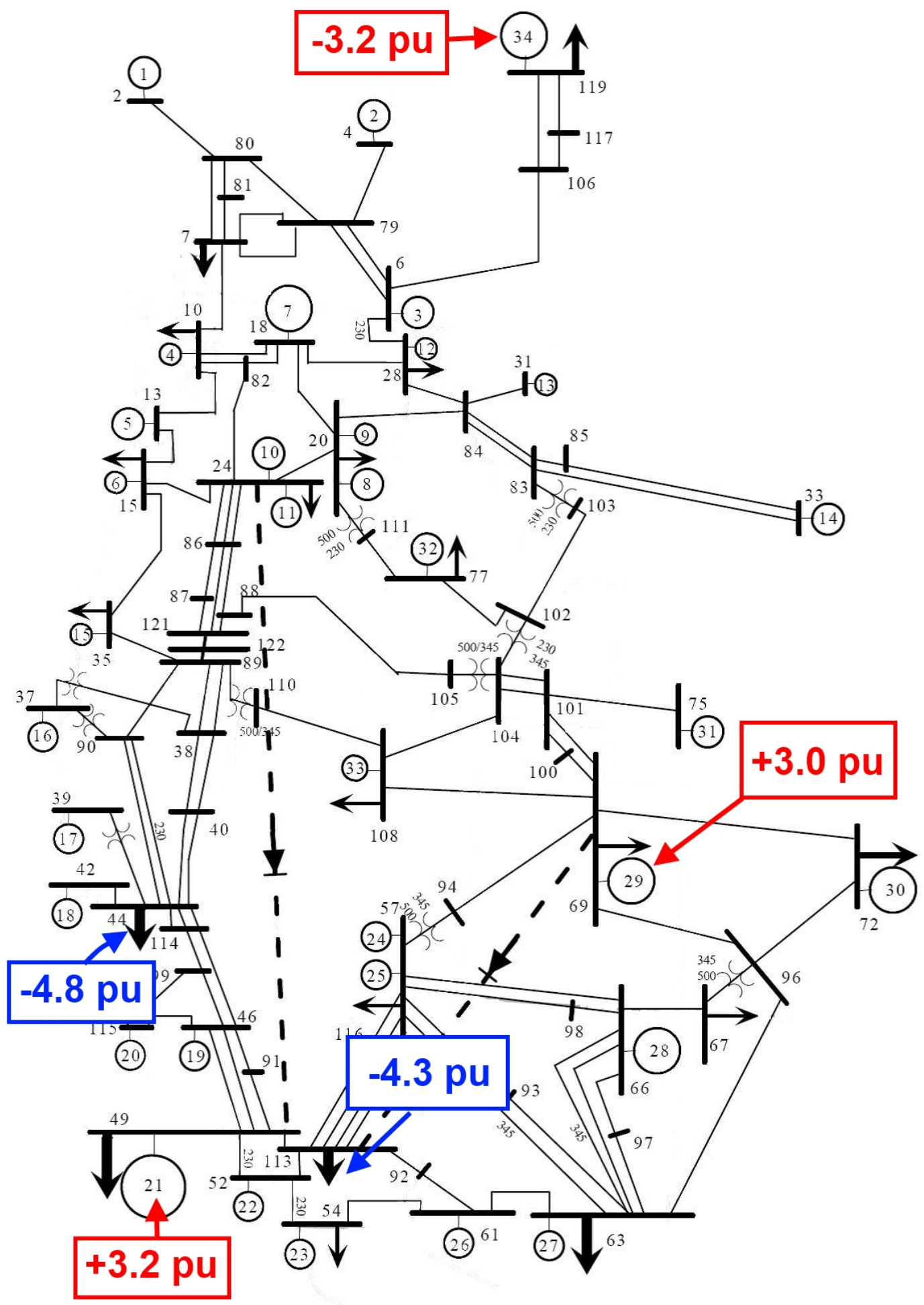

Figure 33. Geographical distribution of top five MANGO controls.

Like all other ANN applications, the challenge with this ANN-based approach lies at extrapolating the trained ANN for untrained scenarios such as topology changes. The work is ongoing in studying the impact of topology changes on the ANN-based sensitivity approach. Section 8.0 reports the progress. 


\subsection{MANGO Method 3: Operation Procedure Derivation from Decision Tree}

This section aims at designing the action of generation re-dispatch and proposes a method to derive operational procedures to reach a desired damping ratio. This method involves a sensitivity-based approach to selecting critical generators for re-dispatch and a decision-tree (DT)-assisted method to approximate the security boundary, which helps to find the shortest distance to adjust power flow to reach a desired level of damping enhancement.

The DT method has been widely used in many fields such as medical diagnosis, financial and statistical analyses and power systems. Application of DTs in transient and voltage stability assessment can be found in Wehenkel et al. [46]-[51]. In more recent efforts, methods of transient stability constrained optimal power flow using generator rescheduling have been developed in Genc et al. [52]-[53]. In this work, decision tree is trained using the method in Breiman et al. [54], where linear combination of attributes is used to identify the security boundary. These different applications of the decision tree method exhibit data and classification qualities similar to the MANGO problem. This makes the extension of the DT method to helping determine generator re-dispatch a natural course for investigation.

\subsection{Decision-Tree-Based Method for Improving Damping of Inter-area Oscillations}

\subsubsection{Main Procedure}

The basic concept of the proposed methodology is to derive operational rules is depicted in Figure 34, with the following steps. More details regarding the design of steps 2)-5) are provided in the following subsections. 


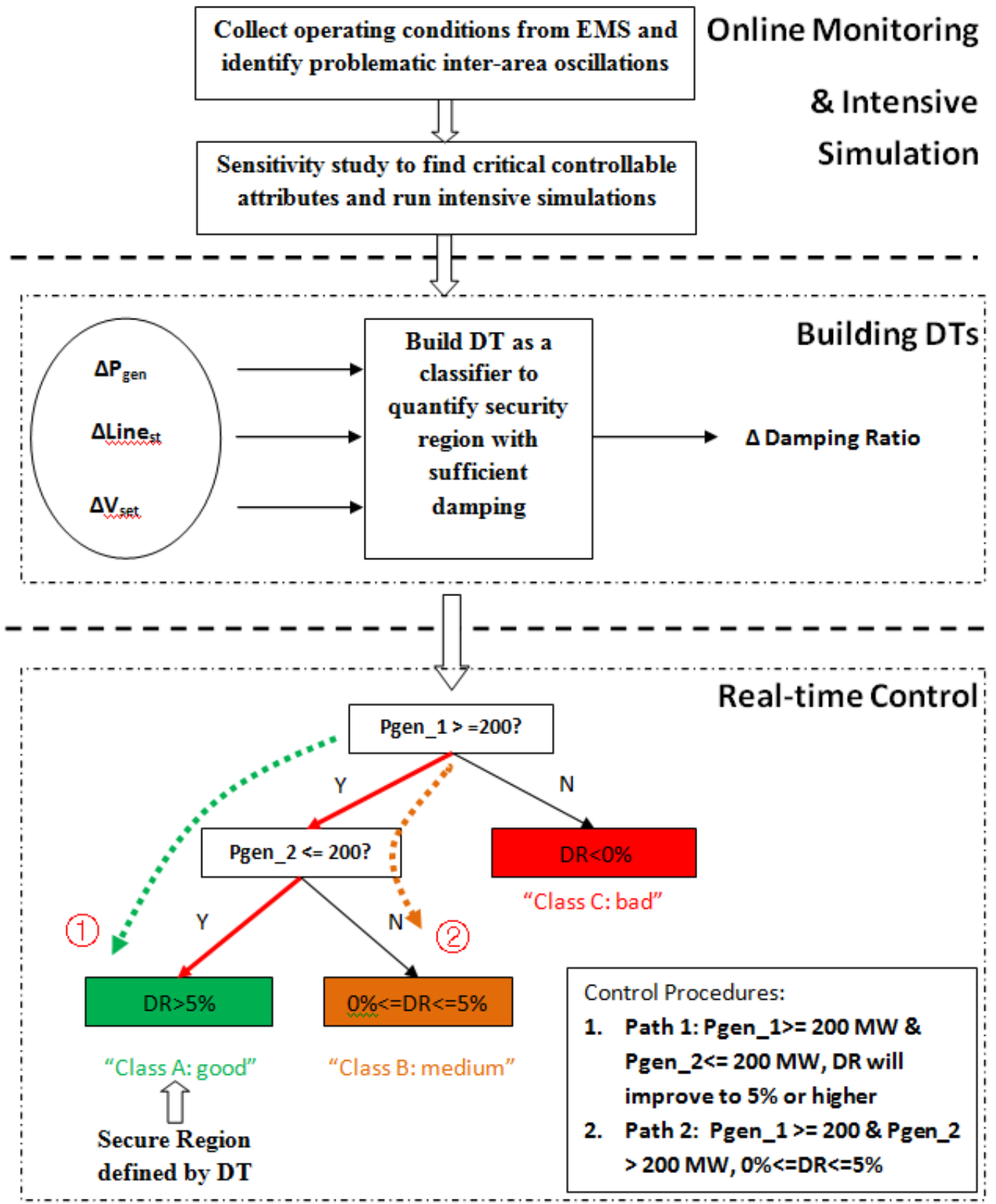

Figure 34. Decision-tree-based method to improve damping of inter-area oscillations

1) In a real-time setting, system status is periodically monitored by energy management system (EMS), with a time interval of several seconds to minutes. Problematic inter-area oscillation modes can be identified using methods such as ModeMeter techniques. If the damping ratio (DR) of the oscillation mode is sufficiently high, there is no need to take any action, skip the following steps until a low damping situation (e.g., $<5 \%$ ) is detected. Otherwise, the current operating condition is used for intensive simulation to design the optimal control scheme.

2) Select a list of candidate controllable attributes that are available for adjustment based on the current operating condition, e.g., generators for active power re-dispatch. Conduct the sensitivity analysis of Section 5 to identify the most effective generator pairs (or groups) to improve the DR of the particular oscillation mode. 
3) Specify the adjustment range and step size for each generator from step ii, and perform intensive simulations on different combination of generator re-dispatch. For each simulation case, the DR value is calculated and recorded. A knowledge base is therefore created, with the target to be the level of DR (secure or insecure) and the attributes as the adjusted generator active power outputs.

4) Build decision-tree model on the created knowledge base and derive security boundaries in terms of the acceptable level of DR, expressed by the DT rules as shown in Figure 34.

5) Using decision-tree rules from step iv and the ranges for generation adjustment from step iii as boundary conditions, solve an optimization problem with the objective to be the shortest distance from the current operating condition to the security boundaries describing the desired damping ratio. The global optimal solution will provide effective operational rules to improve DR to the desired level.

6) Validate the control procedures in the simulation platform before the obtained operational rules are applied to the real power grid. Return to step 1) for real-time damping monitoring and repeat the whole process if the low damping condition continues.

\subsubsection{Overview of the Test System}

A simplified WECC system model is used to test the proposed methodology and explain the detailed procedures. This network model consists of 179 buses, 29 generators, 104 loads, 40 fixed shunt elements, 203 transmission lines, and 60 two-winding transformers. The single line diagram is shown in Figure 35. At base case, the total system load is 60,785.41 MW and 15,351.25 MVAr. Also at the base case, the modal properties of the system are given in Table 9. As with the previous systems, initial studies focused on one inter-area mode of interest. In this case, the $0.3759-\mathrm{Hz}$ mode was selected for study.

Table 9. Selected Modal Properties of 179-bus System Model

\begin{tabular}{|c|c|}
\hline Frequency (Hz) & Damping Ratio (\%) \\
\hline 0.3759 & 4.89 \\
\hline 0.5778 & 2.16 \\
\hline
\end{tabular}




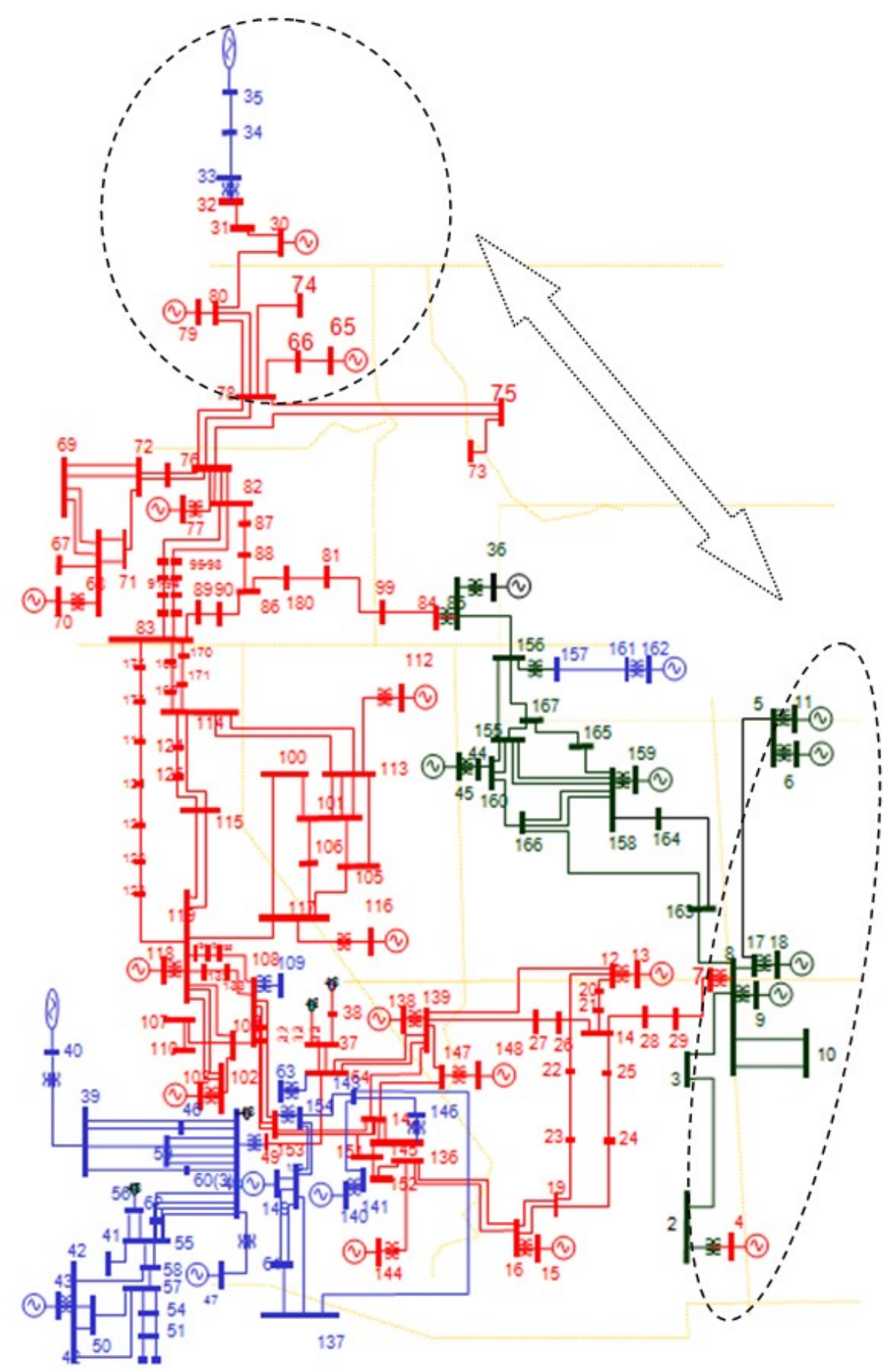

Figure 35. Single-line diagram of 179-bus simplified WECC system

The dynamic model of this network includes generators, excitation systems, governors, and PSSs. For the base case, eigen-analysis was performed in the package, Dynamic Security Assessment Tools (DSA Tools), developed by Powertech Labs [45]. One critical mode of inter-area oscillation with DR less than $5 \%$ is detected, with the frequency of $0.3759 \mathrm{~Hz}$ and a DR of $4.89 \%$. The scatter plot of this mode shape, together with the participating generators, is shown in Figure 36. For this particular mode, two groups of generators are found oscillating against each other, which are also shown in the dashed ellipses in Figure 35. This study will focus on designing the optimal operational procedures to mitigate this critical interarea oscillation mode. 


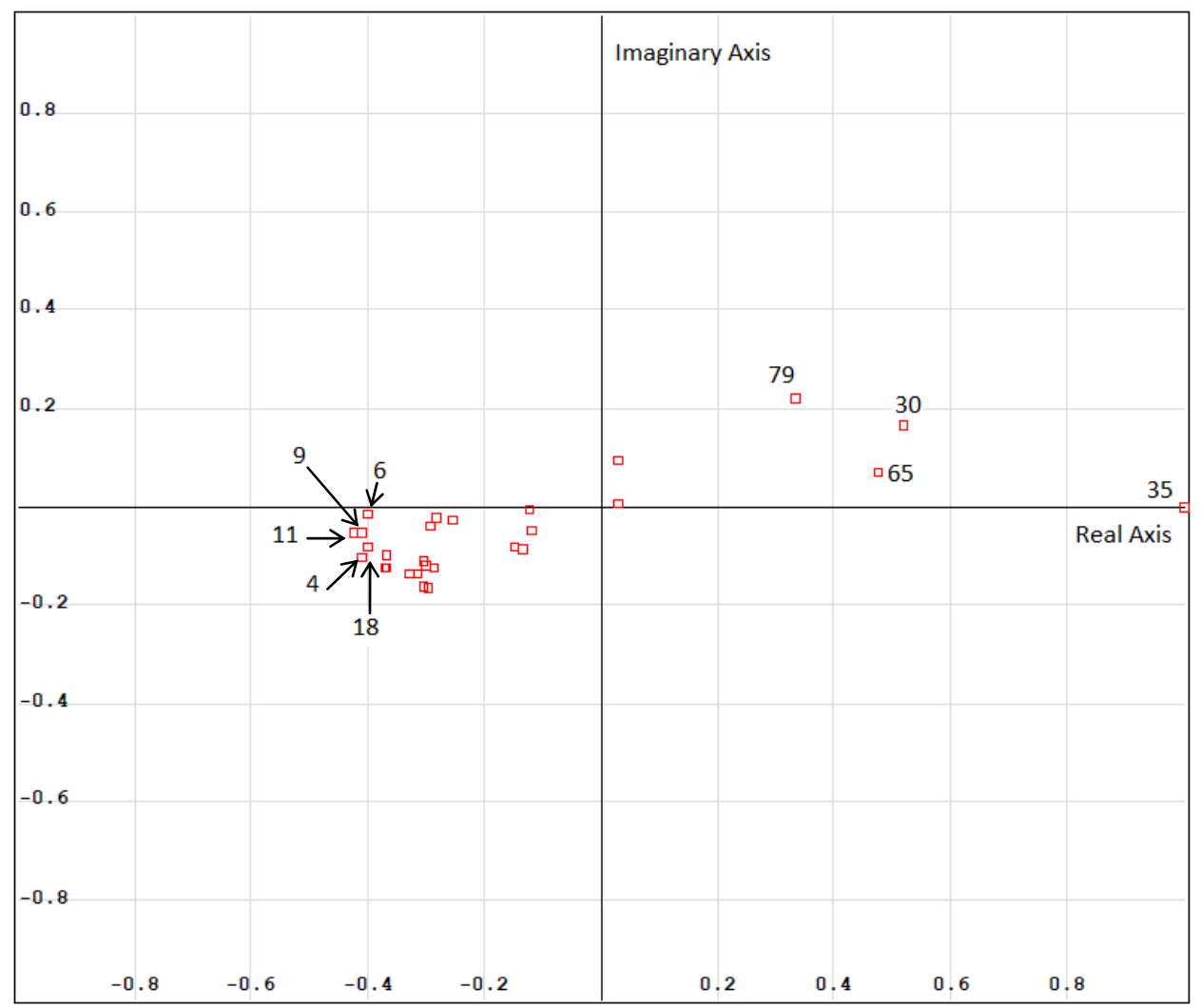

Figure 36. Mode shape of the $0.3759-\mathrm{Hz}$ oscillation in the simplified 179-bus WECC system

\subsubsection{Selection of Critical Generators Using Modal Sensitivity}

Selection of effective generators for active power re-dispatch is an important step to ensure DR enhancement. The sensitivity-based approach proposed in Sections 5.1 and 5.2 is used here to identify the most effective generators that can contribute to the desired DR with the least re-dispatch effort. The eigenvalue sensitivity equation is revisited here

$$
\frac{\partial \lambda}{\partial p}=\boldsymbol{\Psi} \frac{\Delta \mathbf{A}}{\Delta p} \boldsymbol{\Phi}
$$

This modal sensitivity method is applied to the simplified WECC system by adjusting the active power output of each generator, one at a time (ranging from -100 MW to $100 \mathrm{MW}$, with an interval of $20 \mathrm{MW}$ ), with the exception of the swing bus. The swing bus is used to absorb the power imbalance caused by the generation adjustment. The results of the $0.3759-\mathrm{Hz}$ modal sensitivity with respect to generator active power output are shown in Figure 37. The most sensitive generators are also marked in Figure 37, where increasing generation of machines 140, 43, 149 and others provide a positive effect in improving DR; whereas generators 65,79 and 30 provide a negative impact. 


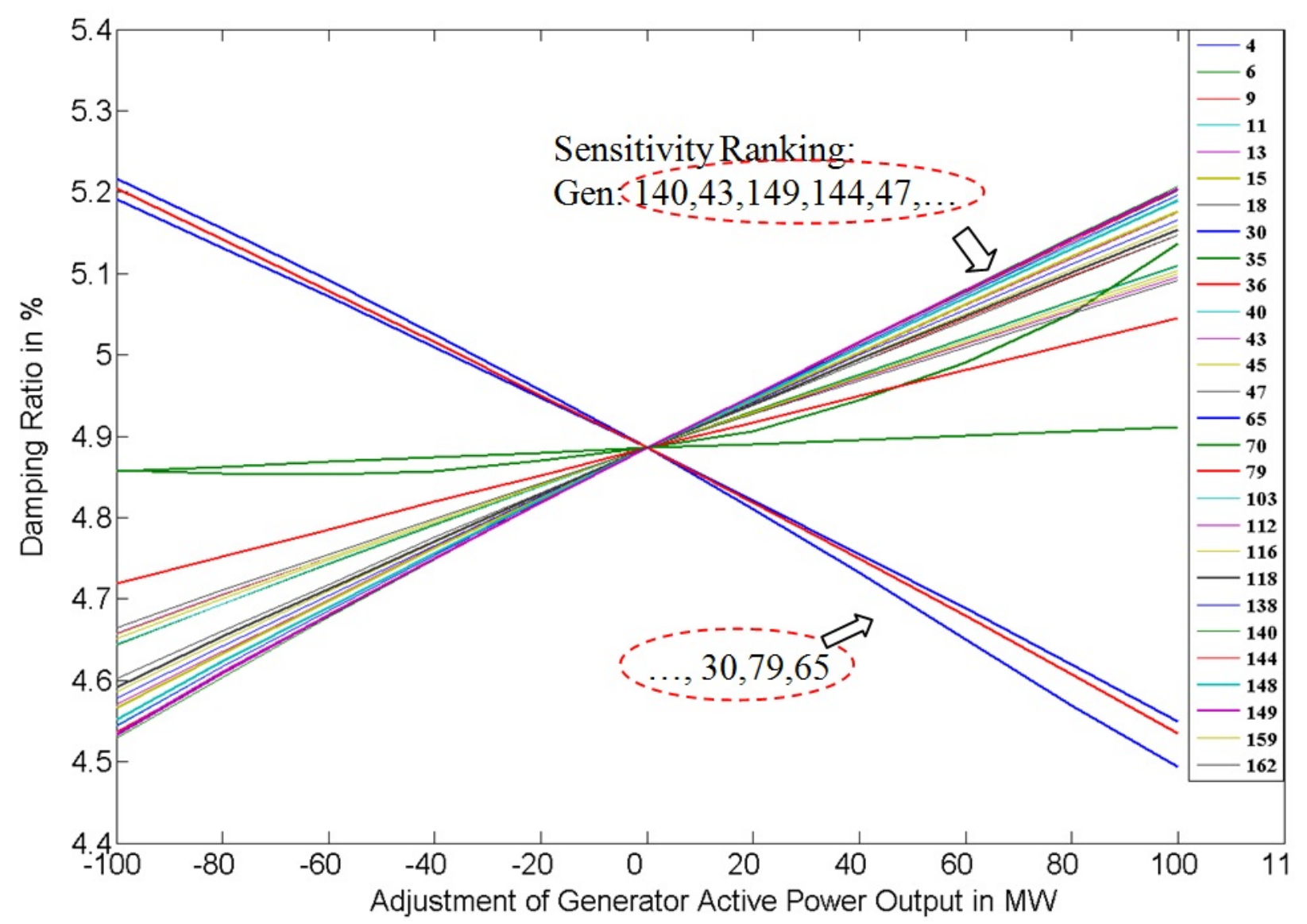

Figure 37. Sensitivity of the DR of the $0.3759 \mathrm{~Hz}$ mode with respect to generator output

This sensitivity study provides insights to guide operating condition adjustment to improve DR. To achieve the most impact, the pair of generators with the largest sensitivity difference should be selected for generation re-dispatch (e.g., increase generation of generator 140 and decrease generator 65). In the following study, generators 140, 43, 149 and 65 are selected for generation re-dispatch for the ease of displaying the optimization process. Selection of generators can be easily extended to a higher dimension without loss of generality. Two interesting observations are also obtained from this study:

i. Almost all of the sensitivity curves exhibit a near-linear pattern around the base case in Figure 37. It forms the basis that the most sensitive generators can be adjusted in a relatively larger range to improve DR.

ii. The most sensitive pairs of generators for generation re-dispatch are not the ones that contribute the most to the $0.3759-\mathrm{Hz}$ inter-area oscillation mode, as shown in Figure 36 (i.e., generators 11, 4 and 35$)$.

\subsubsection{Operational Procedure Using the Decision-Tree Method}

From the sensitivity analysis results, several critical generators are selected regarding the direction of generation adjustment (either increasing active power or decreasing active power). However, the quantity of generation adjustment is not provided. To reach a desired DR, one simple solution could use the trial and error method. This technique involves adjusting the most sensitive generator pair gradually and 
checking the DR value simultaneously, until the adjusted generator pair hits the upper or lower limit. If the desired DR is not met, start to adjust a less sensitive generator pair until the DR requirement is met. However, this method may not be the optimal solution, in terms of adjustment efforts and/or economic costs. Therefore, a decision-tree-assisted approach is presented here to formulate an optimization problem to find the shortest distance (least generation re-dispatch) between the current operating condition and the desired level of DR.

Decision tree is a supervised machine-learning tool for solving classification problems in a highdimensional data space. It provides the capability of classifying an objective (i.e., damping ratio) using the most effective attributes (i.e., generation) that are related to the objective. An example of DT is shown in the bottom third of Figure 34. Typically, there are two types of nodes in a DT: the internal node with two (or more) successors, and the terminal node without any successor. The internal node selects the most effective splitting rule (using either single attribute or combination of attributes) to split the data space (i.e., $\mathrm{P}_{\text {gen__ }}>=200$ ), whereas the terminal node denotes a sub-region for a class (i.e., DR larger than 5\%). A unique feature of DT is that it is possible to derive classifying rules that define sub-regions for different classes. It can derive security boundaries for a desired DR that can be directly used as linear inequality constraints in formulating the optimization problem. The formulation of the optimization problem is detailed in the subsequent subsections.

\section{Generation of Knowledge Base}

For a vector of selected generators, $\boldsymbol{P}_{\boldsymbol{G}}=\left[P_{1}, P_{2}, \ldots, P_{n}\right]$, specify the adjustment range and sampling interval of each generator by system operators:

$$
P_{i \min } \leq P_{i} \leq P_{i \max }
$$

For machines $\left[P_{1}, P_{2}, \ldots, P_{n-1}\right]$, perform an intensive search in the space with a dimension of $n-1$, so that

$$
\sum_{i=1}^{n} P_{i}=\sum_{i=1}^{n} P_{i_{-} 0}
$$

where $P_{i_{-}}$represents the initial active power output of machine $i$. The last machine, $n$, which is dependent on the first $n-1$ machines, is used to balance the change so that the sum of generation is kept constant. This step is to ensure that generation re-dispatch has the minimum impact on the rest of the system, especially the swing bus. One assumption is made here that the change in total power loss caused by different generation re-dispatch schemes is negligible compared to the amount of power adjustment. For each generator re-dispatch scheme with different combination of generation levels, perform the power flow study, run eigenvalue analysis, and record the DR. A threshold of DR needs to be defined by operators to classify the adjusted operating conditions. As an example, if $\mathrm{DR}>=8 \%$ is the target, all the operating conditions meeting this requirement are labeled "secure"; otherwise, an "insecure" label is assigned. Thus, a knowledge base is created for training decision trees. The objective is the damping ratio, and the attributes collect the generator active power output from the $n-1$ machines. 


\section{Mathematical Expression}

The optimization problem to find the shortest distance to a desired DR is defined below:

$$
\begin{aligned}
\text { minimize } & \sqrt{\sum_{i=1}^{\mathrm{n}}\left(P_{i}-P_{i_{-} 0}\right)^{2}} \\
\text { subject to } & \sum_{i=1}^{n} P_{i}=C \\
& P_{i \min } \leq P_{i} \leq P_{i \max } \\
& h\left(P_{i}\right) \leq 0, \quad i=1,2, \ldots, n-1
\end{aligned}
$$

where $C$ is a constant, equal to the sum of initial power outputs of the $n$ selected generators. $h\left(P_{i}\right)$ represents the inequality constraints derived from the specific paths reaching one terminal node labeled "secure". The objective function of this optimization is to minimize the Euclidean distance between the initial operating condition and the surface that separates the "secure" points from the "insecure" ones. For a DT with $k$ terminal nodes labeled as "secure", the entire data space is divided into $k$ sub-regions, each of which is characterized by the paths to each terminal node. Because any sub-region is convex with linear boundary, the local optimal solution for this sub-region is expected to lie on the constraint boundary. The global optimal solution can easily be reached as the one with the minimum objective function value by comparing the local optimal solutions for the $k$ secure sub-regions.

\subsection{Simulation Results}

The proposed method to find the optimal operational procedures is applied to the simplified WECC 179bus system. The four selected generators from sensitivity study in Section 7.2.3 are listed in Table 10, which also shows the direction of power adjustment and the adjustable range.

Table 10. Selected Generators for Generation Re-dispatch

\begin{tabular}{|c|c|c|}
\hline Generator & Initial P output & Adjustable Range \\
\hline 140 & $3195 \mathrm{MW}$ & $3195 \rightarrow 4473 \mathrm{MW}$ \\
\hline 43 & $325 \mathrm{MW}$ & $325 \rightarrow 455 \mathrm{MW}$ \\
\hline 149 & $2200 \mathrm{MW}$ & $2200 \rightarrow 3080 \mathrm{MW}$ \\
\hline 65 & $2910 \mathrm{MW}$ & $2910 \rightarrow 0 \mathrm{MW}$ \\
\hline
\end{tabular}

During the creation of knowledge base, the generation levels are adjusted within each generator's limits, with an incremental step of 5\% of the initial power output. The last generator (No. 65) is used to balance the total generation as long as the adjusted power falls within its acceptable range. All the other generator outputs are kept constant. Therefore, there are 729 operating conditions generated and eigenvalue analysis is performed in the DSA Tools on these operating conditions to form a knowledge base. The impact of generation re-dispatch on the $0.3759-\mathrm{Hz}$ oscillation mode is shown in Figure 38. 


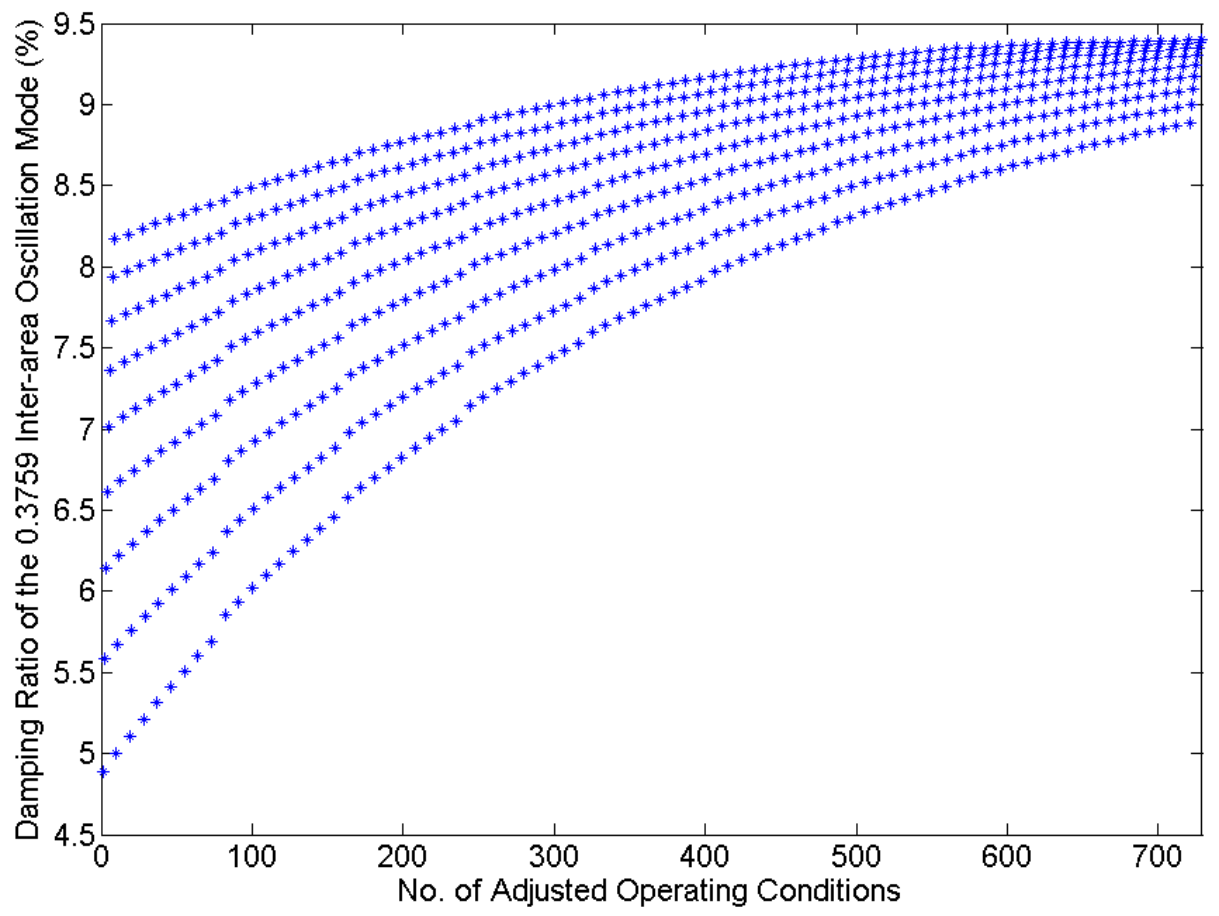

Figure 38. Effect of generation re-dispatch in improving DR

From Figure 38, all the adjusted operating conditions have shown a positive effect in improving DR, as expected. It attributes to the correct adjustment direction determined by the modal sensitivity analysis results. The maximum damping ratio of the $0.3759-\mathrm{Hz}$ mode is $9.4 \%$. It is also expected that higher damping ratio can be attained when more generators with a wider range of adjustment are included in the generation re-dispatch process. A threshold of $8 \%$ is arbitrarily chosen as the control objective for DR improvement, and thus 535 operating conditions are labeled as "secure" and the remaining 194 cases are labeled as "insecure". A decision-tree model is then trained for this knowledge base, and the tree structure is shown in Figure 39. This decision tree has a satisfactory performance in the sense that it finds two planes to divide the three-dimensional space into two secure sub-regions. In each secure region, no insecure points exist. The two secure sub-regions are further summarized in Table 11. 


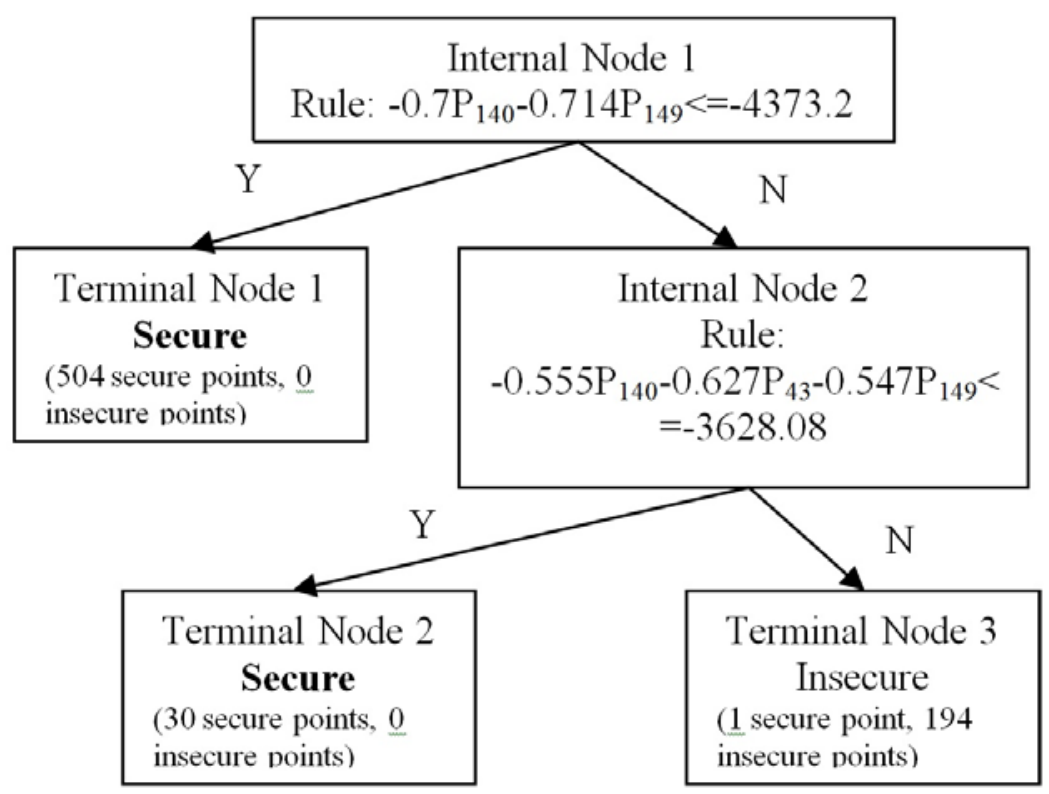

Figure 39. Structure of the decision tree model

Table 11. Secure Sub-regions for Generation Re-dispatch

\begin{tabular}{|c|c|}
\hline $\begin{array}{c}\text { Secure Sub- } \\
\text { Regions }\end{array}$ & Inequality Constraints \\
\hline \multirow{4}{*}{1} & $-0.7 \mathrm{P}_{140}-0.714 \mathrm{P}_{149}<=-4373.2$ \\
\cline { 2 - 3 } & $3195<=\mathrm{P}_{140}<=4473$ \\
\cline { 2 - 3 } & $325<=\mathrm{P}_{43}<=455$ \\
\hline \multirow{4}{*}{2} & $2200<=\mathrm{P}_{149}<=3080$ \\
\cline { 2 - 3 } & $-0.7 \mathrm{P}_{140}-0.714 \mathrm{P}_{149}>-4373.2$ \\
\cline { 2 - 3 } & $-0.555 \mathrm{P}_{140}-0.627 \mathrm{P}_{43}-0.547 \mathrm{P}_{149}<=-$ \\
3628.08 \\
\cline { 2 - 3 } & $3195<=\mathrm{P}_{140}<=4473$ \\
\cline { 2 - 3 } & $325<=\mathrm{P}_{43}<=455$ \\
\cline { 2 - 3 } & $2200<=\mathrm{P}_{149}<=3080$ \\
\hline
\end{tabular}

Together with the objective function:

Minimize

$$
\sqrt{\left(P_{140}-3195\right)^{2}+\left(P_{43}-325\right)^{2}+\left(P_{149}-2200\right)^{2}+\left(P_{65}-2910\right)^{2}}
$$

and the equality constraint:

$$
P_{140}+P_{43}+P_{149}+P_{65}=8630
$$

Two optimization problems are therefore solved in MATLAB and by comparing the two local optimal solutions. It is found that the solution in second sub-region has the shortest distance. As a result, the optimal solution is reached as, $P_{\text {optimal }}$ :

$$
\left(P_{140}=3535.4 \mathrm{MW}, P_{43}=455 \mathrm{MW}, P_{149}=2524 \mathrm{MW} \text { and } P_{65}=2115.6 \mathrm{MW}\right)
$$


The optimal solution and the inequality constraints are depicted in Figure 40 for explaining how this method works. To validate the obtained optimal solution, the controllable rules $\left(\Delta \mathrm{P}_{140}=340.4 \mathrm{MW}\right.$, $\Delta \mathrm{P}_{43}=130 \mathrm{MW}, \Delta \mathrm{P}_{149}=324 \mathrm{MW}, \Delta \mathrm{P}_{65}=-794.4 \mathrm{MW}$ ) are applied to the original operating condition. Simulation result from eigenvalue analysis shows that the damping ratio is increased to $7.98 \%$, which is sufficiently close to the desired $8 \%$. The little error is caused by the linearization process of using DT to approximate the nonlinear surface. To ensure a desired performance, it is suggested to specify a small security margin that allows the boundary to shift toward the secure sub-region. To further evaluate the effectiveness of this method, the concept of "nearest neighbor" is used to find the nearest valid sampling point in the knowledge base with a $\mathrm{DR} \geq 8 \%$. The nearest point, $P_{\text {nearest }}$, is found as

$$
\left(P_{140}=3514.5 \mathrm{MW}, P_{43}=373.7 \mathrm{MW}, P_{149}=2640 \mathrm{MW} \text { and } P_{65}=2101.8 \mathrm{MW}\right)
$$

The distance between $P_{\text {nearest }}$ and $P_{0}$ (the original operating condition) is $975.3588 \mathrm{MW}$, whereas the one between $P_{\text {optimal }}$ and $P_{0}$ is $932.1049 \mathrm{MW}$. However, the $P_{\text {nearest }}$ solution requires fewer generator changes to implement. As a result, the solution obtained from the proposed decision-tree method provides an operation scheme with less efforts than simply identifying the nearest from known operating conditions in the knowledge base.

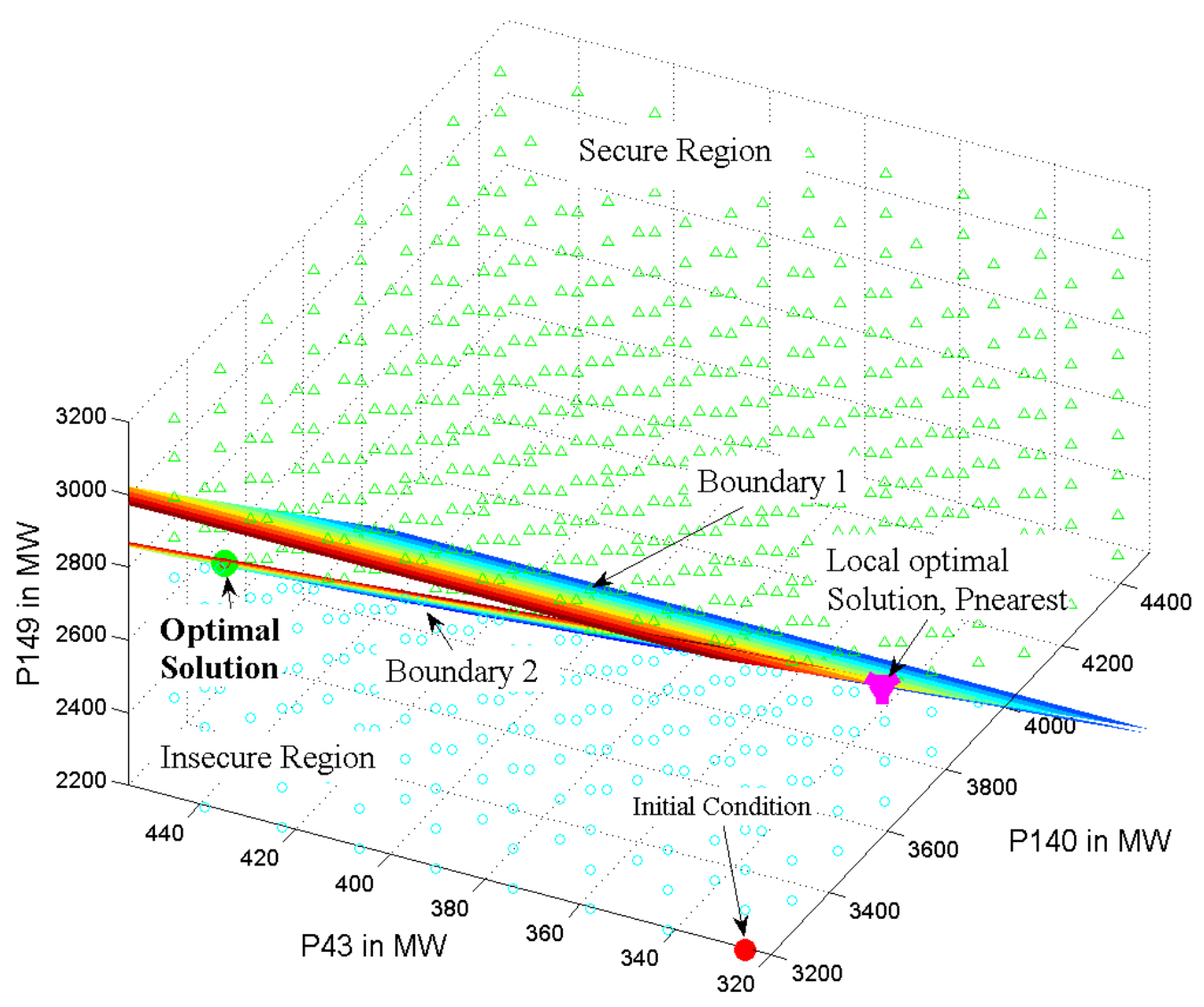

Figure 40. Optimal operational rules to improve DR to $8 \%$ 


\subsection{Summary}

This section proposes a method to improve the damping ratio of problematic inter-area oscillations by generation re-dispatch in a power system. The proposed method includes a sensitivity study to select the most sensitive generators for generation re-dispatch, and a decision tree assisted approach to find the shortest distance (in terms of the least control efforts) to reach a desired damping ratio. This method is tested on the WECC 179-bus system. The initial results demonstrated the validity of the proposed method, and the obtained optimal solution reached the desired performance.

In future work, more studies will be performed to validate the proposed method using the full WECC system model with 16,000 buses and 3000 generators. In addition, sensitivity studies will be performed to evaluate how the optimal control rules may change with different operating conditions; this will pave the way for real-time application of inter-area oscillation mitigation. 


\subsection{Topology Change Impact Analysis}

\subsection{Motivation}

In previous studies, it was assumed that the system topology remains the same. Power grid topology, however, is of great importance in power grid analysis, changing grid characteristics such as power flow patterns as well as system oscillation modes. System topology changes are a common occurrence because of equipment maintenance or failure, economic considerations, faults, reliability requirements, and many other reasons. There is a need, therefore, to investigate the performance of the previously reported MANGO approach while considering the effects of different system topologies. In the following discussions, the effect of system topological changes on system modes (frequency and damping ratio) will be addressed using the 34-machine model shown in Figure 17. The objective is to evaluate and improve the effectiveness of the MANGO framework, while incorporating the effects of power system topological changes.

\subsection{Technical Approach}

To study the impact of different topologies on system modes, a number of simulations were performed for different topological changes. Studies were performed to determine which topological changes had the strongest effect on system modes using the 34-machine model mentioned above. Because the original 34machine model is a reduced order low damping system, each line represents multiple lines in a real system. Attempts were made to modify the topology of the system by removing transmission line objects; however, this often led to divergent or unstable solutions, because each line played an important role. The 34-machine base model was modified to replace each transmission line with two parallel lines of equivalent impedance. A total of 99 new lines were added into this "double-line" system. In this "double-line" model, removing one or more lines from the system represented a less drastic change in topology, and maintained system stability while still modifying the behavior of the system. All studies were performed using this "double-line" model. Input parameters, such as generator output (real power generation) and load demand (real and reactive power consumption), and output parameters, such as mode damping and frequency, were monitored and recorded.

\subsection{Case Studies}

To determine the effects of topological changes, the base system described above was simulated and the frequency and damping ratio (DR) of all modes were monitored. The distribution of all the modes in the base case are shown in Figure 41. To concentrate on a smaller subset of modes of interest, only those between 0.2 and $0.7 \mathrm{~Hz}$ and damping ratios of less than $5 \%$ were monitored. The monitor zone is drawn in Figure 41 as a blue box. This range was chosen because it contained the two least damped modes in the 34-machine model. It can be seen that two modes are dominant in this range at 0.343 and $0.607 \mathrm{~Hz}$. In the simplified model, the $0.343-\mathrm{Hz}$ mode represents the Alberta mode of the full WECC system, while the $0.607-\mathrm{Hz}$ mode represents the Kemano mode. These will be the modes monitored during the following topological studies. 


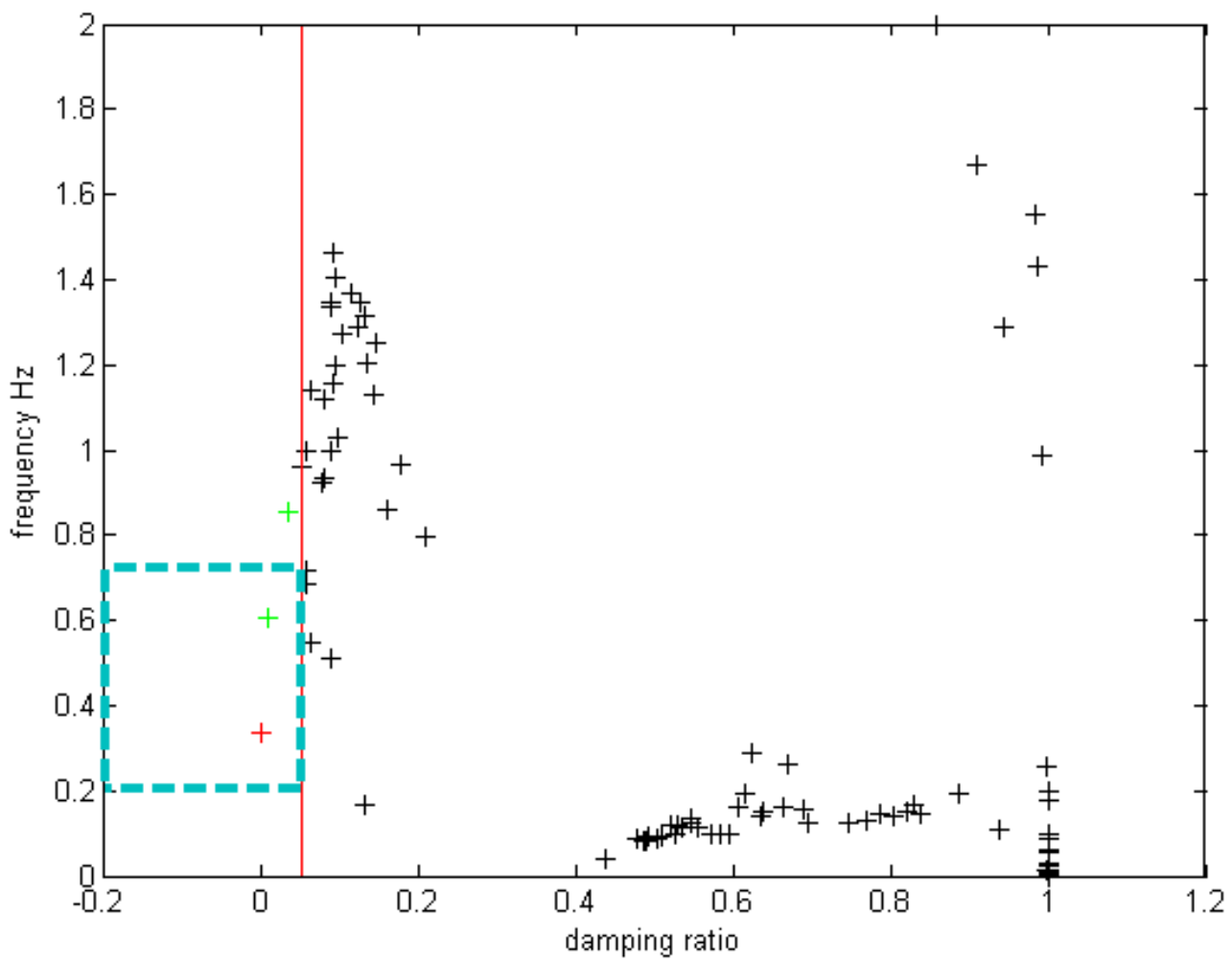

Figure 41. Damping ratio and frequency of all modes in the base case simulation. The blue box represents the monitor zone of system modes.

As indicated earlier, the MATLAB-based Power Systems Toolbox was used to perform eigenvalue analysis for the topological cases. There are total 99 topology change cases in the 34-machine model. Each topology case represents the base case with only one line removed. For each case, a line was successively removed, and the damping ratio and frequency are monitored within the previously specified ranges. The results can be seen in Figure 42, in which the blue cross symbols represent the two base case modes. 


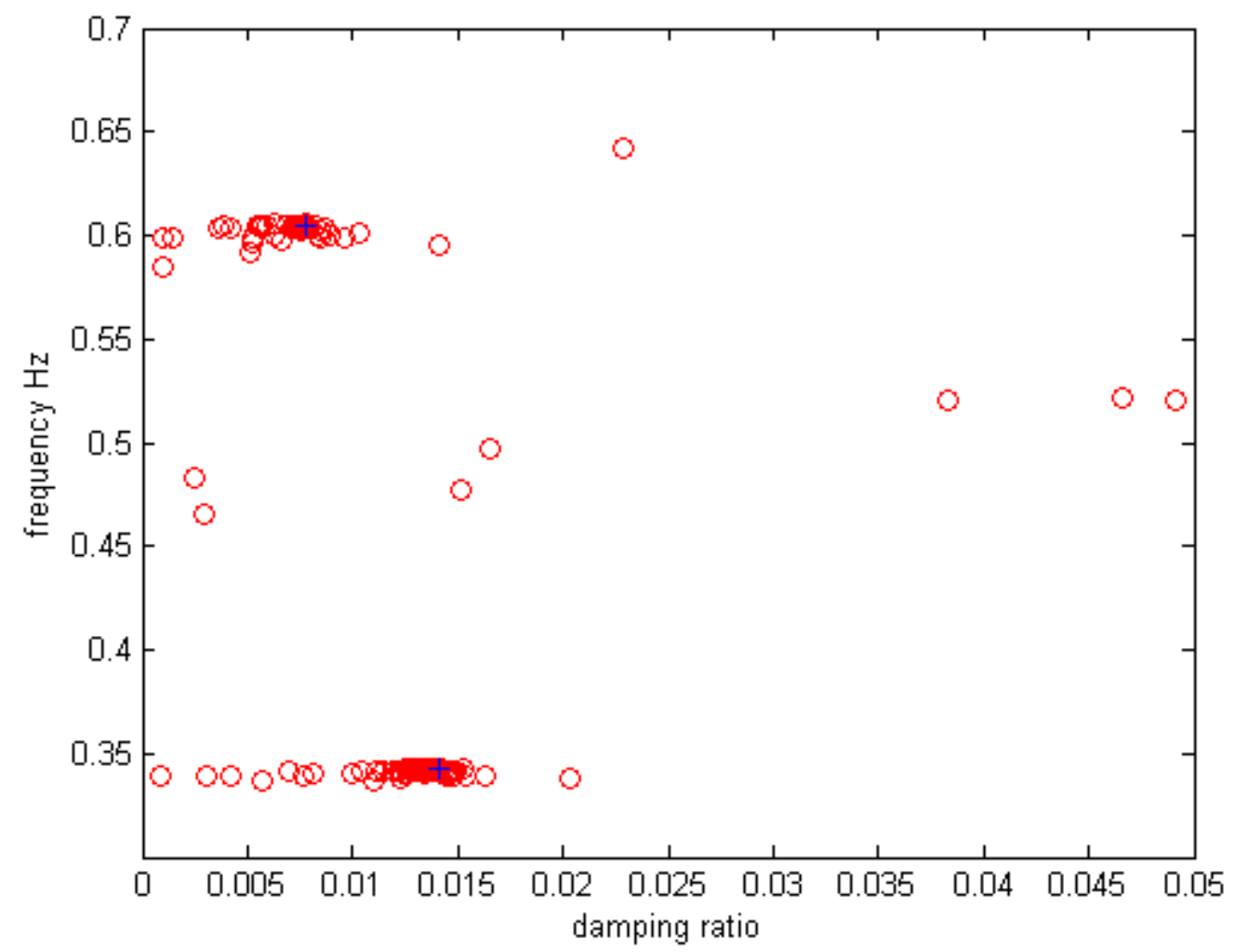

Figure 42. All modes identified in the monitor windows of all topological study cases

The results show that there is a cluster of modes at both $0.6 \mathrm{~Hz}$ and $0.34 \mathrm{~Hz}$. However, it can be noted that there are some "outlier" modes between $0.45 \mathrm{~Hz}$ and $0.55 \mathrm{~Hz}$. To see the modes more clearly, Figure 43 was created. In Figure 43, the red circles represent the $0.34-\mathrm{Hz}$ mode; cyan circles represent the $0.6-\mathrm{Hz}$ modes; blue diamonds represent the cases that three modes can be identified in the monitor window; green squares represent the cases that only one mode can be identified in the monitor window; and the black crosses represent the modes in the base case.

From Figure 43, notice that for $0.34-\mathrm{Hz}$ and $0.6-\mathrm{Hz}$ modes, except for one case (line 18-28, marked by a black arrow), there are only slight variations in the frequencies. However, there are significant changes within the damping ratios for each case, ranging from 0.001 to 0.014 in the $0.6-\mathrm{Hz}$ case and 0.001 to 0.021 in the $0.34-\mathrm{Hz}$ mode. The "outliers" are most likely introduced by the specific topological change, which cause an additional mode to move within the monitoring range. Further research is needed to track mode changes to make sure they are "outlier" modes, and not a valid shift of the $0.34-\mathrm{Hz}$ and $0.60-\mathrm{Hz}$ modes. The lines whose topology change can result in introducing an additional mode in the monitoring window are 33-83, 33-85, 83-84, 31-84, 84-28, 103-102 and 101-75; the lines whose topology change lost one mode are 2-80 and 7-10. The topology change of line 91-49 can result in maximum damping ratio for both $0.34-\mathrm{Hz}(\mathrm{DR}=0.02)$ and $0.60-\mathrm{Hz}(\mathrm{DR}=0.014)$ modes. The topology change of line 104-101 results in minimum damping ratio of 0.0008 for $0.34-\mathrm{Hz}$ mode, and the topology change of line 6-79 will cause a minimum damping ratio of 0.0009 for $0.60-\mathrm{Hz}$ mode. In addition, the line $18-19$ can result in two frequencies of 0.4832 and 0.5991 , with damping ratios equal to 0.0025 and 0.0014 , respectively. The locations of all these lines are shown in Figure 44. 


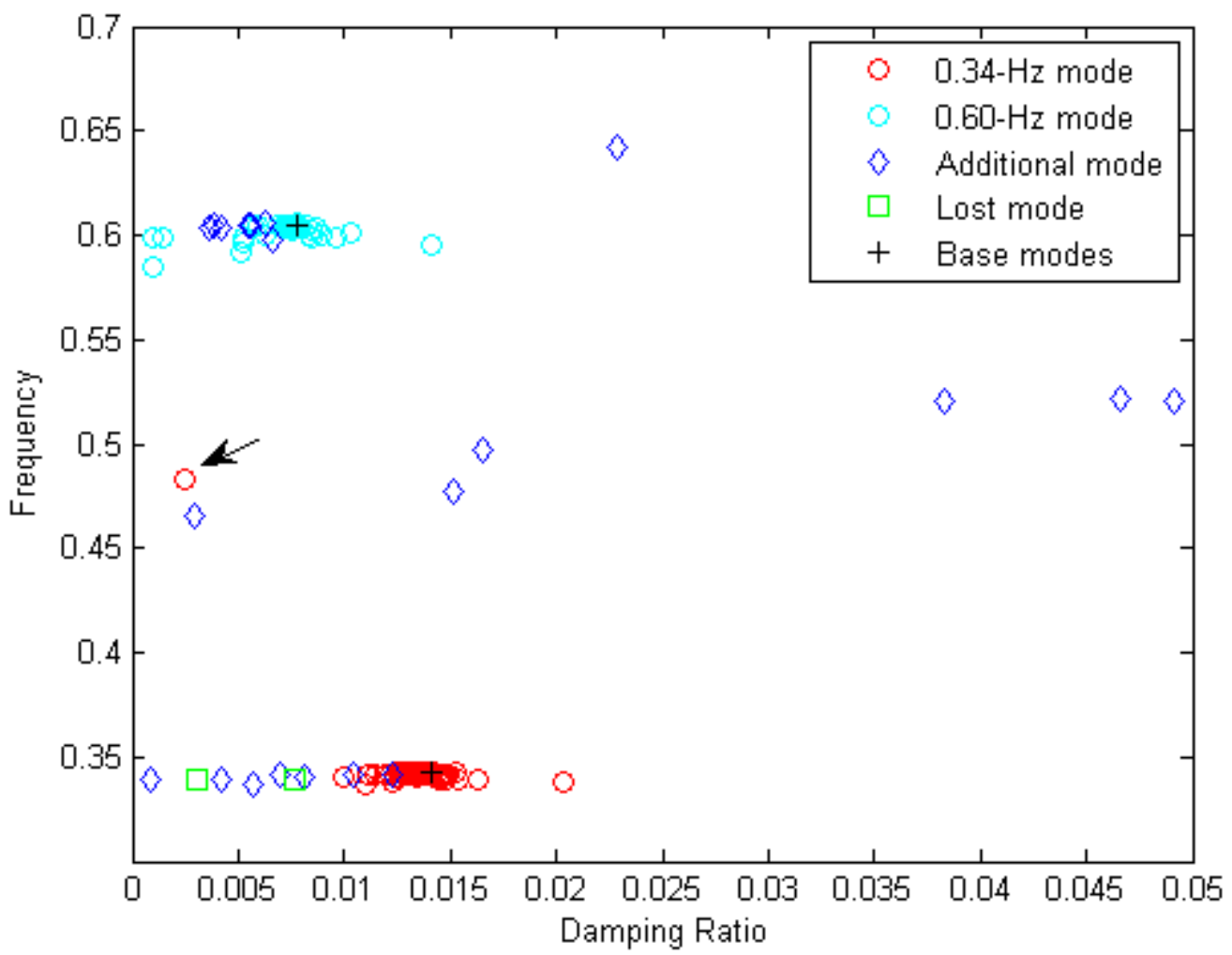

Figure 43. More detailed view of all modes identified in the monitor windows of all topological study cases 


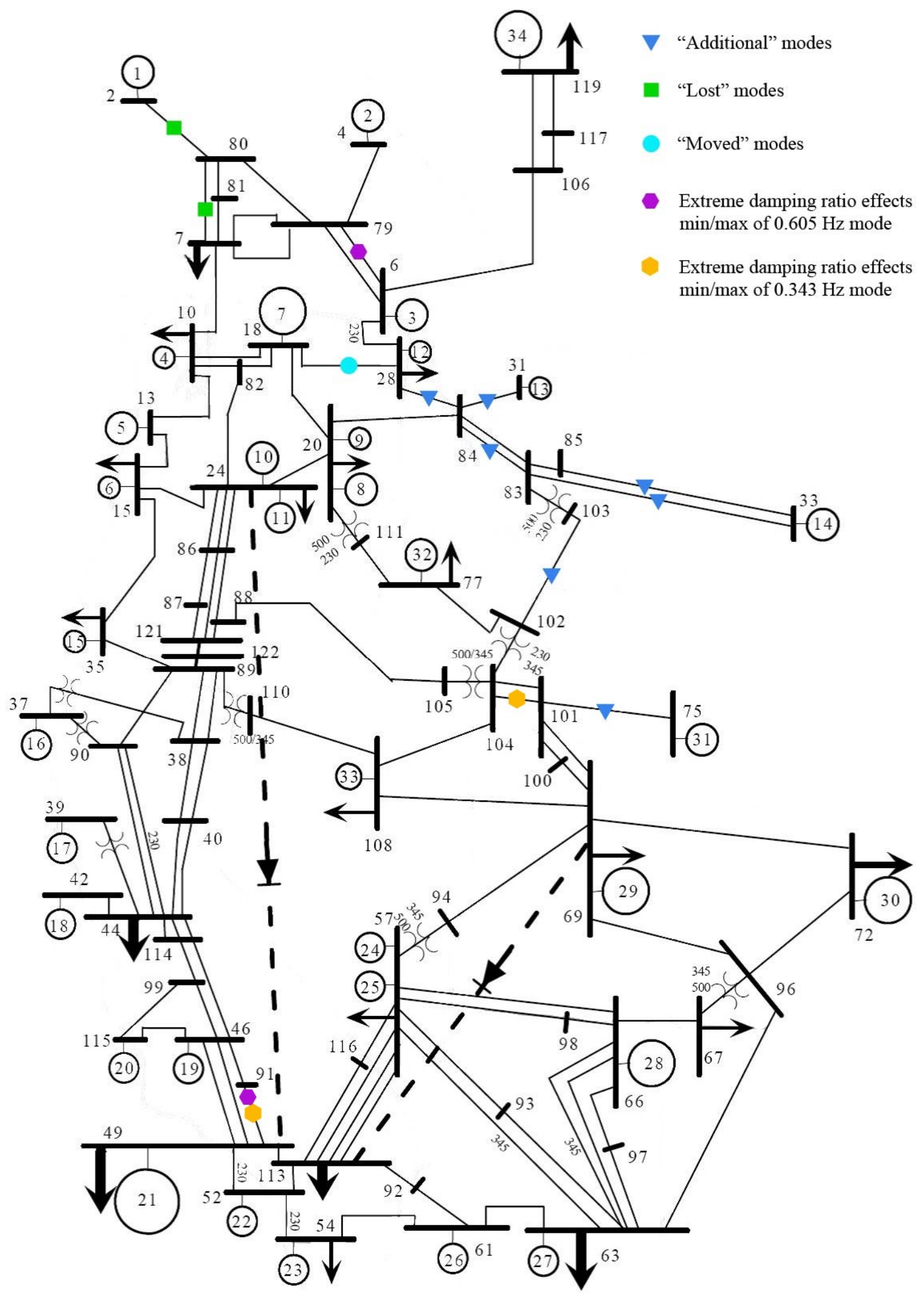

Figure 44. Modified WECC model with removed line cases shown 
The "lost" mode at 0.6-Hz cases (green squares in Figure 43 and Figure 44) are the result of removing one of the tie lines between the Kemano generator and the rest of the system, effectively changing the topology geographically close to the generator that most contributes to the mode. This mode is not necessarily "lost", but more than likely has moved to a different frequency. The "additional" modes (blue diamonds in Figure 43 and Figure 44) are all created by topological changes geographically close to Colstrip, Montana generator, and are the result of reduced damping in other modes in the system. Future work will address these concerns by tracking the "movement" of the modes by incrementally changing the topology of the system, as opposed to step changes in the topology.

\subsection{Summary}

Some preliminary conclusions can be made about the effects of topology changes on the frequency and damping ratio, as applied to the 34-machine model:

1. The cluster of points in Figure 43 at the $0.34-\mathrm{Hz}$ mode show that topological changes have little to no effect on the frequency of the Alberta mode.

2. However, it also shows that topological changes within the system have a direct effect on the damping ratio of the modes. Counter intuitively, in some cases, the removal of a line has the effect of increasing the Alberta mode damping ratio.

3. The cluster of points in Figure 43 at the $0.6-\mathrm{Hz}$ mode show that topological changes have some effect on the frequency of the Kemano mode. However, the variation is less than $0.03 \mathrm{~Hz}$.

4. Similar to the conclusions in the Alberta mode, topological changes within the system also have a direct effect on the damping ratio of the Kemano mode. Once again, cases exist for both increasing and decreasing the damping ratio.

\subsection{Future Work}

Future work in this area will focus on a number of issues raised in the previous analysis. First, a method for tracking the "movement" of modes will be developed to identify the behavior of "outlier" modes. Second, the topological effect on all major system modes will be addressed in a holistic sense (i.e., if the topology change increases the damping ratio of the Alberta mode, how does it affect all the other system modes?). Finally, the feasibility of using this information as an operator actionable item will be explored. 


\subsection{Experience with Simulation Tools for Studying MANGO}

Throughout this research, multitudes of simulation results have been generated. These results have come from sources as simple as a two-area model, to as complex as the 16,000-bus WECC planning case. Each model provides a means of investigating the MANGO approach, as well as validating the expectations. Throughout the project, many different tools have been utilized to produce the results. This section explains the usage of the different tools, particularly as a method for validating the MANGO procedure.

As indicated, several programs were used to generate results for the MANGO project. Despite the different program approaches, the data was often used in a similar manner. Even with different models, the MANGO procedure still required data for investigation, validation, and a path forward to more complicated analysis.

The first requirement fulfilled by different simulation programs in the MANGO project was the generation of simulation data. Sometimes, an underlying theory was developed mathematically or from expert knowledge. Other times, methods evolved from observations in the data. In either scenario, simulation data is required. This data serves as the initial test set for the developed method. For much of the MANGO work developed, this data has simply been a time series output of various points in a power system model, or the eigenvalue results of said model.

Once an approach appears successful on the initial simulation data sets, further simulation data is generated to test the approach. This data works towards validating the method in two ways: validation of the method itself, and validation of its consistency. Validating each technique is of great importance to ensure that it is not only working as expected, but also to provide a basis that the technique can give similar answers for similar operating conditions. If a particular MANGO approach gave significantly different answers for a 90-MW adjustment and a 95-MW adjustment of the same generator, it would warrant further investigation. It may reveal that this is a valid observation (and that the particular generator is extremely sensitive to the metric), or that the technique may not be well suited for generalized data.

Validation of the method itself is relatively straightforward. Sets of simulation data are generated and the particular MANGO approach is applied. As indicated in the earlier example, this can be as simple as varying the output of a single generator and observing the time-series or eigenvalue-analysis results. If the results of the method fall within a specified tolerance of the expectation, the methodology and understanding of that method are valid. If it fails to meet these expectations, the method may require a refinement in its application, or the understanding of the fundamental principles.

The second form of validation, validation of consistency, often takes two approaches as well. The first is the example in the previous paragraph to determine if a method produces similar results over several trials. The other form of consistency validation is to ensure a particular program is providing a reasonable answer. To investigate this requirement, a data set is often analyzed by a second technique. These techniques may not be different from any aspect aside from their originating vendor. For example, the PowerTech Labs time domain simulation incorporates a method for performing ringdown analysis [45], as does the PNNL Dynamic System Identification (DSI) toolbox [56]. Both programs use similar approaches and methods, but two different implementations. For a proper validation, both programs should arrive at similar results for a given data set. 
Data validation is not just restricted to getting the same analysis result. It is also useful in validating the underlying model of a program. This is especially important for the small signal stability aspects of a time-domain simulation, a key portion of any MANGO data set. If an identical model is run through two different programs, such as the Transient Stability Analysis Tool (TSAT) from PowerTech Labs [45] and the Positive Sequence Load Flow (PSLF) program from GE [57], nearly identical results are expected. Figure 45 shows such a validation of the model output for a simulated Chief Joseph dynamic brake insertion using a full WECC system model. When the results of the two programs are relatively matched, as they are in Figure 45, the associated outputs are trusted as reasonable bases for that particular MANGO approach.

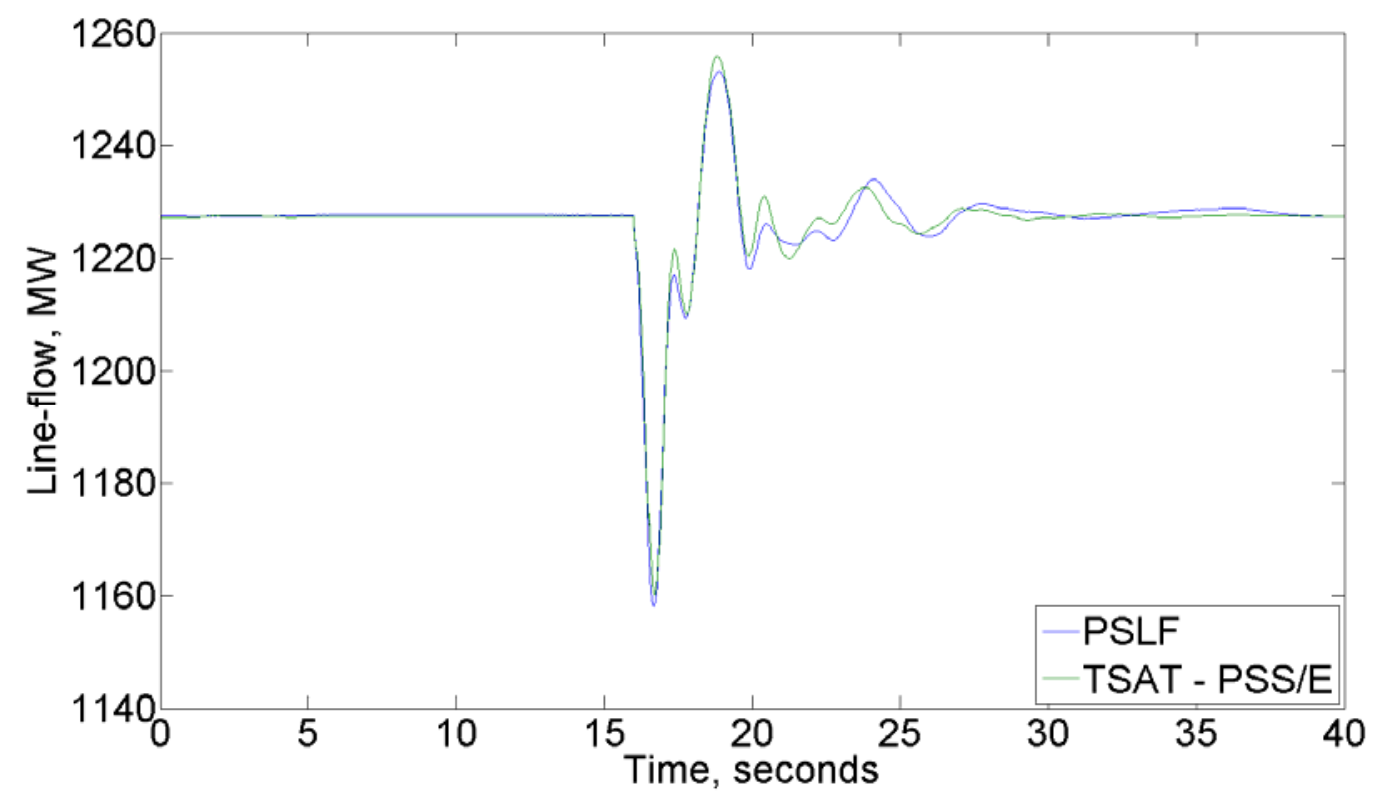

Figure 45. Simulated Chief Joseph dynamic brake insertion

Finally, different programs were utilized in the MANGO evaluations to simply handle different model sizes. A lot of the initial work began with the MATLAB-based Power System Toolbox (PST) [14]. However, simulations of the full WECC planning case were not possible under this toolbox, so other programs were needed. To simulate the full 16,000 bus planning case, PowerTech Lab's Dynamic Security Assessment Tools (DSATools) and GE's PSLF program were utilized. These two commercialgrade programs allow the MANGO investigations to proceed to larger, more realistic models.

Regardless of the program used, the validation step represents one of the most important stages in testing the different MANGO methodologies. Like many classic characterization and pattern recognition patterns, there is always concern of "over-fitting" a model or technique so it performs really well on one data set, but completely fails for more generic data [58][59]. Through the use of different model sizes, as well as different programs to evaluate these models, the research group is ensuring that the MANGO research continues to be applicable to a variety of power system models and conditions. This helps build not only confidence in the method, but also helps maintain the research direction of eventually integrating the MANGO procedure into normal grid operations. 


\subsection{Conclusion and Future Work}

Based on the effect of operating points on modal damping, a MANGO procedure was proposed for improving small signal stability through operating point adjustment. Extensive simulation studies show that damping ratios can be controlled by operators through adjustment of operating parameters such as generation re-dispatch, or load reduction as a last resort. Damping ratios decrease consistently with the increase of overall system stress levels. At the same stress level (i.e., same total system load), inter-area oscillation modes can be controlled by adjusting generation patterns to reduce flow on the interconnecting tie line(s). The effectiveness of the MANGO control is dependent on specific locations where the adjustment is applied. The MANGO procedure consists of three major steps:

1. Recognition - operator recognizes the need for operating point adjustment through on-line ModeMeter monitoring;

2. Implementation - operator implements the adjustment per recommendations by the MANGO approach; and

3. Evaluation - operator evaluates the effectiveness of the adjustment using ModeMeter and repeats the procedure, if necessary.

As the first stage, the MANGO procedure is a measurement-based and operator-in-the-loop procedure. Practical implementation is envisioned to be achieved by integrating MANGO recommendations into existing operating procedures, per NERC and WECC standards. The E-tag system is one such implementation mechanism. The MANGO model can be updated according to the current measurement and mode estimation results. Operators are included in the loop to bring in expert knowledge. In the future, after the confidence and accuracy of MANGO model is built up, it is expected that the automatic, closed-loop control will be introduced to speed up the implementation and avoid human errors. The automatic process can be integrated into a remedial action scheme (RAS) system or a special protection system (SPS).

Both steps 1 and 3 rely on a good ModeMeter to estimate the current modes, while step 2 builds on modal sensitivity, i.e., the relationship of oscillation modes and operating parameters. The relationship is generally nonlinear, and it is impractical to derive a closed-form analytical solution for this relationship. Calculating sensitivity from the system model is not applicable because the model is usually not able to reflect real-time operating conditions. Therefore, this work has been primarily centered on estimating modal sensitivity from real-time measurement. Eigenvalue-theory-based sensitivity has been studied and concluded to be not applicable for real-time estimation. Many items in the eigenvalue-sensitivity equations cannot be estimated with regular phasor measurements. Three approaches for estimating modal sensitivities are then proposed and studied to approximate the sensitivity relationship. These approaches are relative sensitivity estimation, ANN-based nonlinear mapping, and decision-tree-based rule derivation. The relative sensitivity is formulated using least square principles in the form that can be estimated directly from measurement. The testing has been carried out with a medium-sized system. The results indicate how strong generation output relates to the damping, giving clear guidance on which generator needs to be adjusted to improve damping. ANN-based nonlinear mapping has been studied, and it can successfully approximate the modal sensitivity from testing results with medium-size systems. 
Also, a decision-tree based method is studied to summarize the relationship between operator controllable variables and modes.

System topology changes the inherent dynamics in a power system, and thus changes the modal properties. Topology analysis is conducted to characterize the impact of topology change in oscillation modes. As applied to the 34-machine model, most topology changes have significantly more impact on mode damping than on mode frequency, while a few result in a large shift in modal frequency. The key is how to include topology information in the MANGO procedure. Topology impacts the MANGO procedure in two aspects: as input, it may significantly change the sensitivity relationship between operation parameters and mode damping, and as output, topology adjustment can be another option for damping control, as shown in the results with the 34-machine model. This merits further studies.

To pave the way to full-WECC MANGO application, extensive simulation studies have been conducted with commercialized software capable of performing simulation with large systems. A number of problems with models and simulation tools have been solved during this process. More than 1000 cases with randomly adjusted generation outputs were created using an eigenvalue analysis tool. The data sets from these cases will be used to test the modal sensitivity estimation methods in the full-WECC system application. These data will also be further analyzed to derive empirical relationship between damping and operating parameters. This empirical relationship can be used to validate the three MANGO methods in characterizing modal sensitivity, and also as an interim approach for damping control.

In summary, significant progress has been made. A MANGO procedure has been established with practical considerations. The key step in the procedure is the modal sensitivity. Three approaches - direct modal sensitivity estimation, ANN-based nonlinear mapping, and a decision-tree method - have been formulated and heavily studied with promising results from a medium-size system. Impact of topology change on damping has been identified using the ANN-based nonlinear mapping. The simulation studies were conducted with commercialized software, and the resulting experience and data pave the road for large-scale MANGO application. Further work will continue the development of modal sensitivity estimation and application, specifically including work on:

1. Enhance the understanding of the relationship between operating points and mode damping for a practical large system (e.g., the WECC system);

2. Enhance the understanding of the influence of topology changes on major mode;

3. Test and refine the MANGO methods with a larger model;

4. Study the impact of mode meter accuracy on modal sensitivity estimation; and

5. Identify potential implementation strategies with operating procedures. 


\subsection{References}

[1]. CIGRE Technical Brochure, "Analysis and Control of Power Systems Oscillations," CIGRE Task Force 38.01.07, December 1996.

[2]. Kosterev, D.N., C.W. Taylor, and W. A. Mittelstadt, "Model Validation for the August 10, 1996 WSCC System Outage," IEEE Transactions on Power Systems, vol. 14, no. 3, pp. $967-$ 979, August 1997.

[3]. Hauer, J.F., H. Lee, J. Burns, and R. Baker, "Preliminary Analysis of Western System Oscillation Event on June 4, 2003: BPA and Canada," Working Note for the WECC Disturbance Monitoring Work Group, July 7, 2003.

[4]. Hauer, J.F. "Preliminary Examination of the Alberta Trip on August 4, 2000," Working Note for the WECC Modeling and Validation Work Group, June 20, 2002.

[5]. Hauer, J.F. and J.E. Dagle, "Review of Recent Reliability Issues and System Events, “ PNNL13150, prepared for the U.S. Department of Energy Transmission Reliability Program by the Consortium for Electric Reliability Solutions (CERTS), Pacfic Northwest National Laboratory, Richland, WA, December 1999.

[6]. U.S.-Canada Outage Task Force, "Final Report on the August 14, 2003 Blackout in the United States and Canada: Causes and Recommendations," technical report, April 5, 2004.

[7]. Kundur, P. Power System Stability and Control, McGraw-Hill Inc., New York, NY, 1994.

[8]. Hauer, J.F. and J. R. Hunt, in association with the WSCC System Oscillations Work Groups, $V$ Symposium of Specialists in Electric Operational and Expansion Planning (SEPOPE), Recife (PE), Brazil, May 19-24, 1996.

[9]. Zhou, N., D. Trudnowski, J. Pierre, and W. Mittelstadt, "Electromechanical Mode Online Estimation using Regularized Robust RLS Methods," IEEE Transactions on Power Systems, vol. 23, no. 4, pp 1670-1680, Nov. 2008.

[10]. Hauer, J.F., D. J. Trudnowski, and J.D. DeSteese, "WAMS Analysis Tool for Tracking of Oscillatory Dynamics," in Proceedings of the IEEE Power Engineering Society General Meeting 2007, Tampa, FL, June 24-28, 2007.

[11]. Ostojic, D.R., "Stabilization of Multimodal Electromechanical Oscillations by Coordinated Application of Power System Stabilizers," IEEE Transactions on Power Systems, vol. 6, no. 4, pp. 1439-1445, Nov. 1991.

[12]. Ilea, V., A. Berizzi, and M. Eremia, "Damping Inter-area Oscillations by FACTS Devices," 2009 44th International Universities Power Engineering Conference (UPEC 2009), p 5 pp., 2009.

[13]. Chung, C.Y.1; Wang, K.W.; Tse, C.T.; Bian, X.Y.; David, A.K, "Probabilistic eigenvalue sensitivity analysis and PSS design in multimachine systems," IEEE Transactions on Power Systems, vol. 18, no. 4, pp. 1439-45, Nov. 2003.

[14]. Chow, J.H., and K. W. Cheung, "A Toolbox for Power System Dynamics and Control Engineering Education and Research," IEEE Transactions on Power Systems, vol.7, no.4, pp.1559-1564, Nov 1992.

[15]. Hauer, J.F., C. J. Demeure, and L.L. Scharf, "Initial Results in Prony Analysis of Power System Response Signals," IEEE Transactions on Power Systems, vol. 5, no. 1, pp. 80-89, Feb. 1990. 
[16]. Pierre, J.W., D. J. Trudnowski, and M. K. Donnelly, "Initial Results in Electromechanical Mode Identification from Ambient Data," IEEE Transactions on Power Systems, vol. 12, no. 3, pp. 1245-1251, Aug. 1997.

[17]. Kamwa, I., G. Trudel, and L. Gerin-Lajoie, "Low-order Black-box Models for Control System Design in Large Power Systems," IEEE Transactions on Power Systems, vol. 11, no. 1, pp. 303-311, Feb. 1996.

[18]. Zhou, N., J. Pierre, and J. Hauer, "Initial Results in Power System Identification from Injected Probing Signals using a Subspace Method," IEEE Transactions on Power Systems, vol. 21, no. 3, pp. 1296-1302, Aug. 2006.

[19]. Messina, A. and V. Vittal, "Nonlinear, Non-stationary Analysis of Interarea Oscillations via Hilbert Spectral Analysis," IEEE Transactions on Power Systems, vol. 21, no. 3, pp. 12341241, Aug. 2006.

[20]. Sanchez-Gasca, J. J. and J. H. Chow, "Performance Comparison of Three Identification Methods for the Analysis of Electromechanical Oscillations," IEEE Transactions on Power Systems, vol. 14, no. 3, pp. 995-1001, Aug. 1999.

[21]. Trudnowski, D. J., J. M. Johnson, and J. F. Hauer, "Making Prony Analysis more Accurate using Multiple Signals," IEEE Transactions on Power Systems, vol. 14, no. 1, pp. 226-231, Feb. 1999.

[22]. Trudnowski, D., "Estimating Electromechanical Mode Shape from Synchrophasor Measurements," IEEE Transactions on Power Systems, vol. 23, no. 3, pp. 1188-1195, Aug. 2008.

[23]. Weis, R., A. Balasubramanian, and. J. W. Pierre, “Combining Least Mean Squares Adaptive Filter and Auto-regressive Block Processing Techniques for Estimating Low-Frequency Electromechanical Modes in Power Systems," in Proceedings of the IEEE Power Engineering Society General Meeting 2006, Montreal, Quebec, Canada, Jun. 2006.

[24]. Zhou N., J. Pierre, D. Trudnowski, and R. Guttromson, "Robust RLS Methods for On-line Estimation of Power System Electromechanical Modes," IEEE Transactions on Power Systems, vol 22., no. 3, pp. 1240-1249, Aug. 2007.

[25]. Zhou N., Z. Huang, F. Tuffner, J. Pierre, and S. Jin, "Automatic Implementation of Prony Analysis for Electromechanical Mode Identification from Phasor Measurements," in Proceedings of the IEEE Power and Energy Society General Meeting, Minneapolis, MN, USA, July 25-29, 2010.

[26]. Zhou N., D. Trudnowski, and J. Pierre, "Mode Initialization for On-line Estimation of Power System Electromechanical Modes," in Proceedings of the 2009 Power Systems Conference and Exposition (PSCE), Seattle, WA, USA, Mar. 15-19, 2009.

[27]. Pai, M.A., D.P. Sen-Gupta, and K.R.Padiyar, Small Signal Analysis of Power Systems, Alpha Science International, Ltd., Harrow, Middlesex, UK, 2004.

[28]. Trudnowski, D., M. Donnely, and E. Lightner, "Power-System Frequency and Stability Control using Decentralized Intelligent Loads," Proceedings of the 2005/2006 IEEE Power and Energy Society Transmission and Distribution Conference and Exposition, Dallas, TX, pp. 1453-1459, May 2006.

[29]. North American Electric Reliability Corporation (NERC), (October 2007) "Standard TOP004-1 — Transmission Operations", Accessed September 30, 2010. [Online]. Available: http:// www.nerc.com/files/TOP-004-1.pdf. 
[30]. North American Electric Reliability Corporation (NERC), (March 26, 2009) "Standard TOP004-3 - Real-time Transmission Operations", Accessed September 30, 2010. [Online]. Available: http:// www.nerc.com/files/TOP-004-2.pdf.

[31]. North American Electric Reliability Corporation (NERC), (April 2005) "Standard TOP-007-0 - Reporting SOL and IROL Violations", Accessed September 30, 2010. [Online]. Available: http://www.nerc.com/files/TOP-007-0.pdf.

[32]. Western Electricity Coordinating Council (WECC), (March 12, 2008) "WECC Standard TOP007-WECC-1 - System Operating Limits", Accessed September 30, 2010. [Online]. Available: http:// www.wecc.biz/Standards/Pending\%20Standards/TOP-007-WECC-1.pdf.

[33]. North American Electric Reliability Corporation (NERC), (April 2005) "Standard TOP-008-0 - Response to Transmission Limit Violations", Accessed September 30, 2010. [Online]. Available: http:/www.nerc.com/docs/standards/rs/retired/TOP-008-0.pdf.

[34]. North American Electric Reliability Corporation (NERC), (January 2007) "Standard TOP008-1 - Response to Transmission Limit Violations", Accessed September 30, 2010. [Online]. Available: http://www.nerc.com/files/TOP-008-1.pdf.

[35]. North American Electric Reliability Corporation (NERC), (October 23, 2007) "Standard IRO006-4 - Reliability Coordination - Transmission Loading Relief”, Accessed September 30, 2010. [Online]. Available: http://www.nerc.com/files/IRO-006-4.pdf.

[36]. Western Electricity Coordinating Council (WECC), "WECC Standard IRO-STD-006-0 Qualified Path Unscheduled Flow Relief”, Accessed September 30, 2010. [Online]. Available: http://www.nerc.com/files/IRO-STD-006-0.pdf.

[37]. Western Electricity Coordinating Council (WECC), (December 2006) E-Tags, Accessed November 15, 2009. [Online]. Available:

http://www.wecc.biz/committees/StandingCommittees/OC/ISAS/Shared\%20Documents/Form s/AllItems.aspx.

[38]. Tse, C.T. and S. K. Tso, "Modal and Eigenvalue Sensitivity Analyses of Power Oscillations in Interconnected Systems," APSCOM-91. 1991 International Conference on Advances in Power System Control, Operation and Management (Conf. Publ. No.348), p 808-14 vol.2, 1991.

[39]. Smed, T., "Feasible Eigenvalue Sensitivity for Large Power Systems," IEEE Transactions on Power Systems, vol. 8, no. 2, pp. 555-563, May 1993.

[40]. Rommes, J. and N. Martins, "Computing Large-scale System Eigenvalues most Sensitive to Parameter Changes with Applications to Power System Small Signal Stability," IEEE Transactions on Power Systems, vol. 23, no. 2, pp. 434-442, May 2008.

[41]. Huang, Z., N. Zhou, F. Tuffner, Y. Chen, D. Trudnowski, W. Mittelstadt, J. Hauer, and J. Dagle, "Improving Small Signal Stability through Operating Point Adjustment," in Proceedings of the 2010 IEEE Power and Energy Society General Meeting, Minneapolis, MN, USA, July 25-29, 2010.

[42]. Chow, J. and G. Rogers, Power System Toolbox, Cherry Tree Scientific Software, [Online] Available: http://www.ecse.rpi.edu/pst/PST.html, 2000.

[43]. Mathworks, MATLAB User's Manual, The MathWorks, Natick, MA, 2010.

[44]. Demuth, H., M. Beale, and M. Hagan, MATLAB Neural Network Toolbox 6 User's Guide, The MathWorks, Natick, MA, 2009.

[45]. Powertech Labs, DSA Tools, Accessed September 29, 2010, [Online]. Available: http://www.dsatools.com. 
[46]. Wehenkel, L., T. Van Cutsem, and M. Ribbens-Pavella, "An Artificial Intelligence Framework for on-Line Transient Stability Assessment of Power Systems," IEEE Transactions on Power Systems, vol. 4, no. 2, pp. 789 - 800, 1989.

[47]. Wehenkel, L. and M. Pavella, "Decision Trees and Transient Stability of Electric Power Systems," Automatica, vol. 27, no. 1, pp.115-134, Jan., 1991.

[48]. Wehenkel, L, M. Pavella, E. Euxibie, and B. Heilbronn, "Decision Tree Based Transient Stability Method A Case Study," IEEE Transactions on Power Systems, vol. 9, no. 1, pp.459469, Feb. 1994.

[49]. Rovnyak, S., S. Kretsinger, J. Thorp, and D. Brown, "Decision Trees for Real-time Transient Stability Prediction," IEEE Transactions on Power Systems, vol. 9, no. 3, pp.1417-1426, Aug. 1994.

[50]. Sun, K., S. Likhate, V. Vittal, V. Kolluri, and S. Mandal, “An Online Dynamic Security Assessment Scheme Using Phasor Measurements and Decision Trees," IEEE Transactions on Power Systems, vol. 22, no. 4, pp.1935-1943, Nov. 2007.

[51]. Diao, R., K. Sun, V. Vittal, R. O'Keefe, M. Richardson, N. Bhatt, D. Stradford and S. Sarawgi, "Decision Tree Based Online Voltage Security Assessment Using PMU Measurements," IEEE Transactions on Power Systems, vol. 24, no. 2, pp. 832-839, Apr. 2009.

[52]. Genc, I., R. Diao, and V. Vittal, "Computation of Transient Stability Related Security Regions and Generation Rescheduling Based on Decision Trees," in Proceedings of the IEEE 2010 Power and Energy General Meeting, Minneapolis, MN, USA, July 25-29, 2010.

[53]. Genc, I., R. Diao, V. Vittal, S. Kolluri, and S. Mandal, "Decision Tree-Based Preventive and Corrective Control Applications for Dynamic Security Enhancement in Power Systems," IEEE Transactions on Power Systems, vol. 25, no. 3, pp. 1611-1619, Jul. 2010.

[54]. Breiman, L., J. Friedman, R. A. Olshen and C. J. Stone, Classification and Regression Trees, Wadsworth International Group, 1984.

[55]. Huang, Z., N. Zhou, F. Tuffner, Y. Chen, D. Trudnowski, J. Dagle, and J. Hauer, "MANGO Framework and Its Application for the Western Interconnection," PNNL-18680, Pacific Northwest National Laboratory, Richland, WA, August 2009.

[56]. Pacific Northwest National Laboratory, Dynamic System Identification Toolbox, Accessed Sep. 29, 2010, [Online]. Available:

http://www.naspi.org/toolRepository/tool_details.aspx?tid=54.

[57]. GE Energy, Positive Sequence Load Flow Software, Accessed Sep. 29, 2010, [Online]. Available: http://www.gepower.com/prod_serv/products/utility_software/en/ge_pslf/index.htm.

[58]. Duda, R., P. Hart, and D. Stork, Pattern Classification, $2^{\text {nd }}$ edition, Wiley-Interscience Publications, New York, 2001.

[59]. Ljung, L., System Identification: Theory for the User, 2nd edition, Prentice Hall, Upper Saddle River, New Jersey, 1999.

[60]. C. Carter and D. Trudnowski, "Relations of Electro-Mechanical Damping to Generation Loading in the MinniWECC System," Report no. EE2010-1, Electrical Engineering Dept., Montana Tech of the University of Montana, Sep. 2010. 


\section{Distribution}

No. of

Copies

\# Name

Organization

Address

City, State, ZIP Code
No. of

Copies 


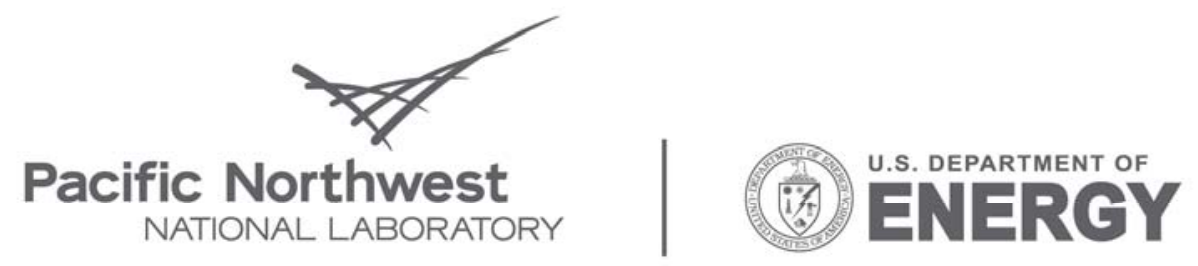

Proudly Operated by Battelle Since 1965

902 Battelle Boulevard

P.O. Box 999

Richland, WA 99352

1-888-375-PNNL (7665)

www.pnl.gov 